\title{
A QUANTUM ELECTRODYNAMICAL FOUNDATION FOR MOLECULAR PHOTONICS
}

\author{
DAVID L. ANDREWS \\ School of Chemical Sciences, University of East Anglia, \\ Norwich, United Kingdom
}

PHILIP ALLCOCK

Department of Physics, University of Bath, Bath, United Kingdom

\section{CONTENTS}

\author{
I. Introduction \\ II. Foundations \\ III. Media Corrections \\ IV. Perturbative Development \\ V. Time Orderings and State Sequences \\ VI. Tensor Representation \\ VII. Construction of Radiation Tensors \\ VIII. Pump Photonics \\ IX. Construction of Molecular Response Tensors \\ X. Damping \\ XI. Index Symmetry and Molecular Structure \\ XII. Two-Level Systems \\ XIII. Optical Coherence in Dispersed Particles \\ XIV. Six-Wave Second-Harmonic Generation \\ XV. Conclusion \\ Acknowledgments \\ References
}




\section{INTRODUCTION}

Molecular photonics has come of age at the threshold of the new millennium. With the main principles of molecule-photon interaction generally well understood, and with laser science mature, it is a field in which we are now witnessing an unparalleled advancement in science and technology, and the realisation of many new and exciting applications. It is nonetheless a field in which the gulf between the two disciplines of chemistry and optics, which represent its molecular and photonic heritage, demands a conceptual and mathematical bridge of sufficient strength to support its progeny. At one extreme, the chemists and materials scientists whose work is increasingly directed toward the devising, synthesis, and characterization of novel photonic materials, need a framework that can accommodate and relate to their insights into the relationships between molecular quantum mechanics, structure, and optical properties. At the other, laser physicists and optical engineers need a vehicle for the furtherance of theory in a form that can reveal the detailed format of the quantum optical parameters that relate to particular materials.

As a theory that addresses the full extent of its molecular photonics remit with the equitable rigour of quantum mechanics, quantum electrodynamics is undoubtedly the tool of choice for this demanding task. In a previous review, one of us has delineated the development of a quantum electrodynamical framework for the generation of optical harmonics in molecular systems [1]. The present work has a rather different focus and is intended to supplement that review, making reference to it but expanding its remit and elaborating on different topics. Theory is cast in a form suitable to address any condensedphase system of independent atoms or molecules, for example liquids, solutions, molecular crystals, or mesoscopically more intricate structures such as membranes. Among other things, this present work focuses on a number of more recent topical issues such as the quantum-optical basis for dissipative and refractive effects, the role of permanent dipole moments, resonance damping, and time-reversal symmetry. Attention is also drawn to a new diagram-based calculational device that appears to offer significant advantages over the traditional time-ordered diagrammatic methods.

\section{FOUNDATIONS}

To fully develop the photonic and material components of quantum-optical response invites the application of quantum electrodynamics (QED). The defining characteristic of this theory is that it addresses every optical interaction in terms of a closed dynamical system where light and matter are treated on an equal footing, each component addressed with full quantum-mechanical rigor. It is a theory whose predictions have been tested to a higher degree of precision 
than any other in modern physics, and that remains unchallenged by the most sophisticated experimental measurements [2]. Even in the noncovariant form commonly employed for dealing with the optical interactions of conventional matter, QED accommodates retardation features associated with the finite time of signal propagation. The success of QED in leading to the correct form of the Casimir-Polder interaction, for example, owes its origin to this intrinsic property of its formulation [3-6]. Indeed, it has recently been shown that even the application of properly retarded classical electrodynamics produces results of significantly different form [7]. In the subjects to be described below, retardation effects are not specifically at issue - and the advantages of a QED foundation, which we shall highlight, are entirely independent of such features. The need to apply QED in order to properly accommodate retardation features in the quantum optics of nanostructures has nonetheless been demonstrated by Chernyak and Mukamel [8]. The interested reader may also find another body of work on resonance energy transfer and cooperative absorption, in which we have described several processes where retardation is a highly significant factor. The primary references to such work can be found elsewhere in reviews of that subject area $[9,10]$.

The familiar semiclassical basis for optical calculations has been compared to the QED method previously [1]. Some of its shortcomings in connection with nonlinear optics and electro-optics have recently been highlighted [11]. Not surprisingly, the semiclassical theory is inconsistent with the general principles of quantum optics, allowing for example the detection of a single photon by two different detectors [12]. The semiclassical invocation of an electric polarization as the oscillating moment of a radiating dipole, coupled with the electric field vector of the ensuing radiation, generally casts the signal amplitude in the form of a sum of contributions associated with physically distinct processes-when it is a fundamental violation of the superposition principle to summarize the amplitudes of transitions between nonidentical sets of initial and final radiation states. Again, the semiclassical polarization formalism does not allow the full incorporation of magnetic and diamagnetic interactions. For example, in a general three-wave interaction mediated by a species that supports E1 ${ }^{2} \mathrm{M} 1$ (two electric dipoles, one magnetic dipole) but not $\mathrm{E} 1^{3}$ channels, the magnetic dipole interaction in the former can be associated with each of the three waves, yet for obvious reasons only two are accommodated in the electric polarization. It has also been remarked that outside of QED there is no formal basis for establishing the gauge transformations that underpin the familiar multipolar description of optical interactions [13,14a,b].

The definitive molecular formulation of quantum electrodynamics established by Power [3] and Craig and Thirunamachandran [15] forms the primary basis for the theory developed below (see also Dalton et al. [16]). This framework provides for direct calculation of the tensor parameters involved in 
linear and nonlinear optical interactions, which naturally emerge from the derivation of observables such as signal intensities. The starting point for such calculations is the QED Hamiltonian for the dynamical system, wherein matter is conventionally described in terms of individual components with distinct electronic integrity and overall electrical neutrality. In the following text we cast theory in a form suitable to address any condensed-phase system of independent atoms or molecules, for example, liquids, solutions, molecular crystals, or even mesoscopically more intricate structures such as membranes. The theory can also be applied to subunits such as ions or chromophores, assuming that it is the transitions in these that dominate the optical response of the medium, so that each ion or chromophore can be treated as the optical representative of a local environment that is itself electrically neutral. For simplicity, the term molecules is used here as an umbrella term for the distinct optical units labeled $\xi$. In multipolar form the system Hamiltonian may then be represented as follows;

$$
H=H_{\mathrm{rad}}+\sum_{\xi} H_{\mathrm{mol}}(\xi)+\sum_{\xi} H_{\mathrm{int}}(\xi)
$$

Here $H_{\text {rad }}$ is the Hamiltonian for the radiation field in vacuo, $H_{\text {mol }}$ the field-free Hamiltonian for molecule $\xi$, and $H_{\text {int }}$ is a term representing molecular interaction with the radiation. It is worth emphasising that the basic simplicity of Eq. (1) specifically results from adoption of the multipolar form of light-matter interaction. This is based on a well-known canonical transformation from the minimal-coupling interaction [17-21]. The procedure results in precise cancellation from the system Hamiltonian of all Coulombic terms, save those intrinsic to the Hamiltonian operators for the component molecules; hence no terms involving intermolecular interactions appear in Eq. (1).

An important implication of developing theory from the full QED Hamiltonian is that neither the eigenstates of $H_{\text {rad }}$ nor those of $H_{\text {mol }}(\xi)$ are stationary states for the system described by it. Thus the presence of the radiation field modifies the form of the molecular wavefunctions, and equally the presence of matter modifies the form of the radiation wavefunctions. Since the Hamiltonian remains the same irrespective of the state of the system, then even when no light is present the coupling still effects a modification of the molecular wavefunctions. This is, for example, manifest in the occurrence of spontaneous emission (luminescence) from isolated molecules in excited states, the lifting of degeneracy between the $2^{2} S_{1 / 2}$ and $2^{2} P_{1 / 2}$ states of atomic hydrogen (the Lamb shift), also the Casimir force between conducting plates, and yet again the corrections responsible for what was once considered the "anomalous" magnetic moment of the electron.

We now consider the detailed nature of the terms in the QED Hamiltonian. The simplest to deal with is the middle term, which denotes a sum of the normal 
nonrelativistic Schrödinger operators $H_{\mathrm{mol}}(\xi)$ for each molecule, the operator counterparts of their classical energies, which need no further elaboration. Equally, the radiation field term $H_{\text {rad }}$ is the operator equivalent of the classical expression for electromagnetic energy - which, recalling the relation $c^{2}=$ $1 /\left(\mu_{0} \varepsilon_{0}\right)$ between the vacuum electric susceptibility $\varepsilon_{0}$ and magnetic permeability $\mu_{0}$, is expressible as

$$
H_{\mathrm{rad}}=\frac{1}{2} \int\left[\varepsilon_{0} \mathbf{e}^{\perp 2}(\mathbf{r})+\mu_{\mathbf{0}}^{-1} \mathbf{b}^{2}(\mathbf{r})\right] \mathrm{d}^{3} \mathbf{r}
$$

Here $\mathbf{e}^{\perp}$ is the fundamental transverse microscopic electric field operator and $\mathbf{b}$ is the corresponding magnetic field operator. The superscript on the electric field operator designate its transverse character with respect to the direction of propagation, redundant in the case of the magnetic field as it is intrinsically transverse, namely, divergence-free, since it arises from the curl of a vector potential field $\mathbf{a}(\mathbf{r})$. Since the electric field also derives from $\mathbf{a}(\mathbf{r})$, we concentrate first on the second-quantized form of this vector potential, which is cast in terms of a summation over radiation modes as follows:

$$
\mathbf{a}^{\perp}(\mathbf{r})=\sum_{\mathbf{k}, \lambda}\left(\frac{\hbar}{2 V \omega \varepsilon_{0}}\right)^{1 / 2}\left[\mathbf{e}_{\mathbf{k}}^{(\lambda)} a_{\mathbf{k}}^{(\lambda)} e^{i \mathbf{k} \cdot \mathbf{r}}+\overline{\mathbf{e}}_{\mathbf{k}}^{(\lambda)} a_{\mathbf{k}}^{\dagger(\lambda)} e^{-i \mathbf{k} \cdot \mathbf{r}}\right]
$$

Here $V$ denotes the quantization volume, and $\mathbf{e}_{\mathbf{k}}^{(\lambda)}$ is the unit polarization vector for the radiation mode characterized by wavevector $\mathbf{k}$, polarization $\lambda$ and circular frequency $\omega=c|\mathbf{k}|$; where it appears, an overbar denotes complex conjugation. The polarization vector is considered a complex quantity specifically to admit the possibility of circular or elliptical polarizations. Associated with each mode $(\mathbf{k}, \lambda)$ are a Hermitian conjugate pair of photon annihilation and creation operators, $a_{\mathbf{k}}^{(\lambda)}$ and $a_{\mathbf{k}}^{\dagger(\lambda)}$, respectively, which operate eigenstates of $H_{\mathrm{rad}}$ with $m(\mathbf{k}, \lambda)$ photons ( $m$ being the mode occupation number) as follows

$$
\begin{aligned}
a_{\mathbf{k}}^{(\lambda)}|m(\mathbf{k}, \lambda)\rangle & =\sqrt{m}|(m-1)(\mathbf{k}, \lambda)\rangle \\
a_{\mathbf{k}}^{\dagger(\lambda)}|m(\mathbf{k}, \lambda)\rangle & =\sqrt{(m+1)}|(m+1)(\mathbf{k}, \lambda)\rangle
\end{aligned}
$$

reducing the number of $(\mathbf{k}, \lambda)$ photons by one in the former case and increasing it by one in the latter. We note in passing that these operators are not forminvariant, meaning that although the same symbols are used in connection with field expansions in the minimal coupling formalism, the operators themselves differ as from those we employ for multipolar coupling, as the radiation states on which they operate also differ when matter is present [22]. 
Our efforts will be repaid if we take pause to examine the properties of the vector potential, and thereby also its derivative fields. The vector potential is self-evidently Hermitian, as befits the status of the field it represents. Its parity with respect to space-inversion is odd, since $P$ operation reverses the sign of $\mathbf{r}$, $\mathbf{e}$, and $\mathbf{k}$. Its character with respect to time-inversion $T$, which is also of interest, is less self-evident. First, this operation gives

$$
\mathbf{a}^{\perp}(\mathbf{r}) \stackrel{T}{\longrightarrow} \sum_{\mathbf{k}, \lambda}\left(\frac{\hbar}{2 V \omega \varepsilon_{0}}\right)^{1 / 2}\left[\overline{\mathbf{e}}_{-\mathbf{k}}^{(\lambda)} a_{-\mathbf{k}}^{(\lambda)} e^{i(-\mathbf{k} \cdot \mathbf{r})}+\mathbf{e}_{-\mathbf{k}}^{(\lambda)} a_{-\mathbf{k}}^{\dagger(\lambda)} e^{-i(-\mathbf{k} \cdot \mathbf{r})}\right]
$$

since it reverses the sign of $\mathbf{k}$ and complex conjugates all numbers. Then, since the sum over the dummy variable $\mathbf{k}$ extends in all directions, it is convertible to a sum over $-\mathbf{k}$, and using the permissible relation $\overline{\mathbf{e}}_{-\mathbf{k}}^{(\lambda)}=-\mathbf{e}_{\mathbf{k}}^{(\lambda)}[23,24]$, we obtain the result that $\mathbf{a}^{\perp}(\mathbf{r})$ is also of odd parity in time. Now, using the source-free result

$$
\mathbf{e}(\mathbf{r})=-\frac{\partial \mathbf{a}(\mathbf{r})}{\partial t}
$$

implemented in the interaction picture where time features explicitly [compare with the later equations (30)-(32)], we obtain the following expression for the electric field operator:

$$
\mathbf{e}^{\perp}(\mathbf{r})=i \sum_{\mathbf{k}, \lambda}\left(\frac{\hbar \omega}{2 V \varepsilon_{0}}\right)^{1 / 2}\left[\mathbf{e}_{\mathbf{k}}^{(\lambda)} a_{\mathbf{k}}^{(\lambda)} e^{i \mathbf{k} \cdot \mathbf{r}}-\overline{\mathbf{e}}_{\mathbf{k}}^{(\lambda)} a_{\mathbf{k}}^{\dagger(\lambda)} e^{-i \mathbf{k} \cdot \mathbf{r}}\right]
$$

Equally, from

$$
\mathbf{b}(\mathbf{r})=\operatorname{curl} \mathbf{a}(\mathbf{r})
$$

we have a magnetic field given by

$$
\mathbf{b}(\mathbf{r})=i \sum_{\mathbf{k}, \lambda}\left(\frac{\hbar \omega \mu_{0}}{2 V}\right)^{1 / 2}\left[\mathbf{b}_{\mathbf{k}}^{(\lambda)} a_{\mathbf{k}}^{(\lambda)} e^{i \mathbf{k} \cdot \mathbf{r}}-\overline{\mathbf{b}}_{\mathbf{k}}^{(\lambda)} a_{\mathbf{k}}^{\dagger(\lambda)} e^{-i \mathbf{k} \cdot \mathbf{r}}\right]
$$

where the complex unit vector $\mathbf{b}_{\mathbf{k}}^{(\lambda)}$ is defined as

$$
\mathbf{b}_{\mathbf{k}}^{(\lambda)}=\hat{\mathbf{k}} \times \mathbf{e}_{\mathbf{k}}^{(\lambda)}
$$

Again, both the electric and magnetic fields are obviously of Hermitian character. What also emerges from the route of their derivation through Eqs. (3), (6), and 
(8) is that the electric field operator is of odd parity with respect to space, and even parity with respect to time; the magnetic field operator is of even parity with respect to space and odd with respect to time.

Employing the preceding field operator expansions enables the radiation Hamiltonian (2) to be recast in a form that more readily identifies its own quantum properties, explicitly featuring the photon creation and annihilation operators:

$$
H_{\mathrm{rad}}=\sum_{\mathbf{k}, \lambda}\left(a_{\mathbf{k}}^{\dagger(\lambda)} a_{\mathbf{k}}^{(\lambda)}+\frac{1}{2}\right) \hbar \omega
$$

The $\frac{1}{2} \hbar \omega$ associated with each radiation mode is the energy associated with the familiar vacuum fluctuations, the origin of spontaneous emission and self-energy corrections. The eigenstates $|m(\mathbf{k}, \lambda)\rangle$ of $H_{\text {rad }}$ are number states; states that more closely model the coherence and other properties of laser light will be introduced later.

To complete the definitions of the terms in Eq. (1), the full expression for the interaction Hamiltonian $H_{\text {int }}(\xi)$, before multipolar decomposition, can be written as follows

$$
\begin{aligned}
H_{\mathrm{int}}(\xi)= & -\varepsilon_{0}^{-1} \int \mathbf{p}^{\perp}(\xi, \mathbf{r}) \cdot \mathbf{d}^{\perp}(\mathbf{r}) d^{3} \mathbf{r}-\int \mathbf{m}(\xi, \mathbf{r}) \cdot \mathbf{b}(\mathbf{r}) d^{3} \mathbf{r} \\
& +\frac{1}{2} \iint O_{i j}\left(\xi, \mathbf{r}, \mathbf{r}^{\prime}\right) b_{i}(\mathbf{r}) b_{j}(\mathbf{r}) d^{3} \mathbf{r} d^{3} \mathbf{r}^{\prime}
\end{aligned}
$$

where $\mathbf{p}^{\perp}(\xi, \mathbf{r})$ is the transverse electric polarization vector field, $\mathbf{m}(\xi, \mathbf{r})$ is the magnetization vector field, and $\mathbf{O}\left(\xi, \mathbf{r}, \mathbf{r}^{\prime}\right)$ is the diamagnetization tensor associated with molecule $\xi$. Each has a multipolar expansion (see, e.g., Refs. 20 and 21) leading to an infinite series of terms, the leading contributions of which provide the leading terms of $H_{\text {int }}(\xi)$ as follows:

$$
H_{\text {int }}(\xi)=-\varepsilon_{0}^{-1} \mu(\xi) \cdot \mathbf{d}^{\perp}\left(\mathbf{R}_{\xi}\right)-\varepsilon_{0}^{-1} Q_{i j}(\xi) \nabla_{i} d_{j}^{\perp}\left(\mathbf{R}_{\xi}\right)-\mathbf{m}(\xi) \cdot \mathbf{b}\left(\mathbf{R}_{\xi}\right)-\cdots
$$

Here $\boldsymbol{\mu}(\xi)$ is the electric dipole (E1) operator for molecule $\xi$ located at position $\mathbf{R}_{\xi}, Q_{i j}(\xi)$ is the corresponding electric quadrupole (E2) operator, and $\mathbf{m}(\xi)$ is the magnetic dipole (M1) operator. The diamagnetization does not contribute to this order of approximation. We also recognize in Eqs. (12) and (13) the microscopic transverse displacement electric field, $\mathbf{d}^{\perp}$, whose quantum operator form will be discussed in the next section. Explicit expressions for the components of the 
leading molecular multipoles are as follows

$$
\begin{aligned}
\mu_{i}(\xi) & =\sum_{\alpha(\xi)} e_{\alpha}\left(\mathbf{q}_{\alpha(\xi)}-\mathbf{R}_{\xi}\right)_{i} \\
Q_{i j}(\xi) & =\frac{1}{2} \sum_{\alpha(\xi)} e_{\alpha}\left[\left(\mathbf{q}_{\alpha(\xi)}-\mathbf{R}_{\xi}\right)_{i}\left(\mathbf{q}_{\alpha(\xi)}-\mathbf{R}_{\xi}\right)_{j}-\frac{1}{3}\left|\mathbf{q}_{\alpha(\xi)}-\mathbf{R}_{\xi}\right| \delta_{i j}\right] \\
m_{i}(\xi) & =\frac{1}{2} \sum_{\alpha(\xi)} e_{\alpha}\left[\left(\mathbf{q}_{\alpha(\xi)}-\mathbf{R}_{\xi}\right) \times \dot{\mathbf{q}}_{\alpha(\xi)}\right]_{i}
\end{aligned}
$$

where summations are taken over each constituent particle $\alpha(\xi)$ of charge $e_{\alpha}$ and position vector $\mathbf{q}_{\alpha}$. In passing it may be noted that employment of the traceless form of the electric quadrupole and higher-order multipoles is consistent with the divergence-free character of the electric displacement field on which the gradient operator, $\nabla$, acts in Eq. (13). In general, each electric multipole (En) is time-even and carries a $(-1)^{n}$ signature for space inversion; the corresponding magnetic multipole $(\mathrm{M} n)$ is time-odd and has $(-1)^{n-1}$ space parity. Hence the time-even, space-even nature of $H_{\text {int }}$ is secure.

The electric dipole term in (13) normally represents the strongest coupling between matter and radiation and is sufficient for the majority of cases, in which the electronic excitations of molecules are restricted to regions significantly smaller than the wavelengths of the radiation engaged. The electric quadrupole and magnetic dipole terms together are then smaller by a factor typically of the order of the fine structure constant $\alpha=\frac{1}{137}$. The leading diamagnetisation contribution is of the order $\alpha^{2}$, and thus comes into play at the same level as electric octupole and magnetic quadrupole interactions. Although in many quantum optical calculations the detailed, multipolar form of the coupling is deemed largely irrelevant, the spatial and temporal symmetries depend crucially on the multipoles involved, as do the magnitudes of the corresponding coupling constants.

\section{MEDIA CORRECTIONS}

The development of the quantum field theory so far has been cast in a form most directly suited for applications in which the material part of the system comprises only those molecules or optical centers involved in the interactions of interest, with no other matter present. More generally in condensed-phase materials, such centers are surrounded by other atoms or molecules whose electronic properties modify the fields experienced (and produced) by those optical centers. To take account of such influences, we introduce the microscopic displacement electric field $\mathbf{d}$. This arises as a direct consequence of working within the multipolar 
formalism and is related to the fundamental electric field $\mathbf{e}$ and microscopic polarization field $\mathbf{p}$ by

$$
\mathbf{d}^{\perp}=\varepsilon_{0} \mathbf{e}^{\perp}+\mathbf{p}^{\perp}
$$

At this stage the molecular and optical properties are neatly entwined. In its semiclassical macroscopic counterpart, Eq. (17) is termed a material equation because of its engagement of a bulk polarization $\mathbf{P}$; the microscopic and bulk polarisations are, for simple cubic systems, related through the succinct expression

$$
\frac{\mathbf{P}}{3}=\mathbf{p}
$$

It is common practice in the semiclassical formalism to incorporate all the ensuing material-induced (Lorentz) field corrections as an integral part of the optical susceptibilities in an ad hoc manner. In using quantum field theory, and considering all interactions to occur through the exchange of transverse photons, it is not necessary to modify the corresponding molecular polarizabilities, if the field operators take full account of the light propagation environment. Then all matter-induced corrections are carried with the displacement field, and the appropriately modified operator automatically accommodates the local field or media effects.

The nature of media effects relates to the fact that, since the microscopic displacement field is the net field to which molecules of the medium are exposed, it corresponds to a fundamental electric field dynamically "dressed" by interaction with the surroundings. The quantized radiation is in consequence described in terms of "dressed photons" or polaritons. A full and rigorous theory of dressed optical interactions using noncovariant molecular quantum electrodynamics is now available [25-27], and its application to energy transfer processes has been delineated in detail [10]. In the present context its deployment leads to a modification of the quantum operators for the auxiliary fields $\mathbf{d}^{\perp}$ and $\mathbf{h}$, which fully account for the influence of the medium-the fundamental fields of course remain unchanged. Expressions for the local displacement electric and the auxiliary magnetic field operators [27], correct for all microscopic interactions, are then as follows

$$
\begin{aligned}
\mathbf{d}^{\perp}(\mathbf{r})= & i \sum_{\mathbf{k}, \lambda, m}\left(\frac{\hbar v_{g}^{(m)} \omega_{k}^{(m)} \varepsilon_{0}}{2 c V n\left(\omega_{k}^{(m)}\right)}\right)^{1 / 2}\left(\frac{\left\{n\left(\omega_{k}^{(m)}\right)\right\}^{2}+2}{3}\right) \\
& \times\left[\mathbf{e}_{\mathbf{k}}^{(\lambda)} P_{\mathbf{k}, m}^{(\lambda)} e^{i(\mathbf{k} \cdot \mathbf{r})}-\overline{\mathbf{e}}_{k}^{(\lambda)} P_{k, m}^{\dagger(\lambda)} e^{-i(\mathbf{k} \cdot \mathbf{r})}\right] \\
\mathbf{h}^{\perp}(\mathbf{r})= & i \sum_{\mathbf{k}, \lambda, m}\left(\frac{\hbar v_{g}^{(m)} \omega_{k}^{(m)} n\left(\omega_{k}^{(m)}\right)}{2 \mu_{0} c V}\right)^{1 / 2}\left[\mathbf{b}_{\mathbf{k}}^{(\lambda)} P_{\mathbf{k}, m}^{(\lambda)} e^{i(\mathbf{k} \cdot \mathbf{r})}-\overline{\mathbf{b}}_{\mathbf{k}}^{(\lambda)} P_{\mathbf{k}, m}^{\dagger(\lambda)} e^{-i(\mathbf{k} \cdot \mathbf{r})}\right]
\end{aligned}
$$


where $\mu_{0}$ is the magnetic permeability of the vacuum $\left(\mu_{0}=1 / \varepsilon_{0} c^{2}\right)$. To fully appreciate these expressions for the new auxiliary field operators, it is expedient to dwell briefly on their key features and elucidate the new symbols which appear in the preceding equations.

Compared with the mode expansions of their fundamental field counterparts, Eqs. (7) and (9), the most obvious difference apparent in Eqs. (19) and (20) relates to the introduction here of additional summations over $m$. This index labels the branches of polariton dispersion and runs from $m=1,2, \ldots M$, where $M=M_{\mathrm{mol}}+1$ and $M_{\mathrm{mol}}$ is the number of molecular frequencies. For example, in a two-level molecular system characterized by a single transition frequency, there are two branches to the dispersion curve. In general, the summations over $\mathbf{k}$ extend to $k \ll 2 \pi / a$, where $a$ is a characteristic intermolecular separation. Consequently the auxiliary operators are properly invoked only when dealing with the propagation and interactions in condensed media of infrared, optical, or ultraviolet light - where a description in terms of refractive index is entirely legitimate. Nonetheless, the theory properly accommodates not only transparent but also dispersive regions where the polariton wavevector and frequency are not linearly related, signifying resonant or near-resonant optical response. It also affords a means for the representation of photonic bandgap materials. Figure 1 illustrates the photonic and exciton-like regions for conventional two-, threeand multilevel systems. The index $m$, which identifies each of the dispersion branches in the general case, has to be incorporated in the definition of the polariton frequency, as given by

$$
\omega_{k}^{(m)}=\frac{c k}{n\left(\omega_{k}^{(m)}\right)}
$$

where several normal frequencies are associated with each value of $k$, again as evident in Fig. 1. The mode expansions (19) and (20) also feature polariton annihilation and creation operators, $P_{\mathbf{k}, m}^{(\lambda)}$ and $P_{\mathbf{k}, m}^{\dagger(\lambda)}$, respectively, with similar properties to their vacuum counterparts of Eqs. (4) and (5). Finally, Eqs. (19) and (20) also feature the group velocity $v_{g}^{(m)}$, defined for each specific polariton mode as

$$
v_{g}^{(m)}=c\left\{\frac{\partial \omega_{k}^{(m)} n\left(\omega_{k}^{(m)}\right)}{\partial \omega_{k}^{(m)}}\right\}^{-1}=\frac{\partial \omega_{k}^{(m)}}{\partial k}
$$

and again incorporating the frequency-dependent refractive index defined as

$$
\left[n\left(\omega_{k}^{(m)}\right)\right]^{2}=1+\frac{\bar{\alpha}\left(\omega_{k}^{(m)}\right) \rho / \varepsilon_{0}}{1-\bar{\alpha}\left(\omega_{k}^{(m)}\right) \rho / 3 \varepsilon_{0}}
$$




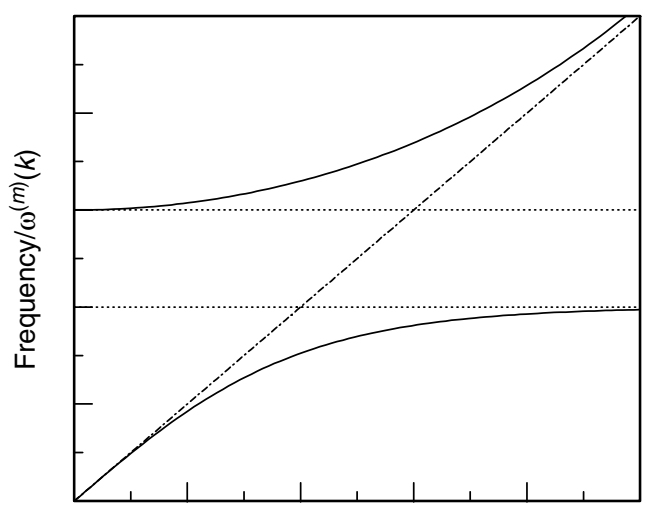

Wave-vector/k

(a)

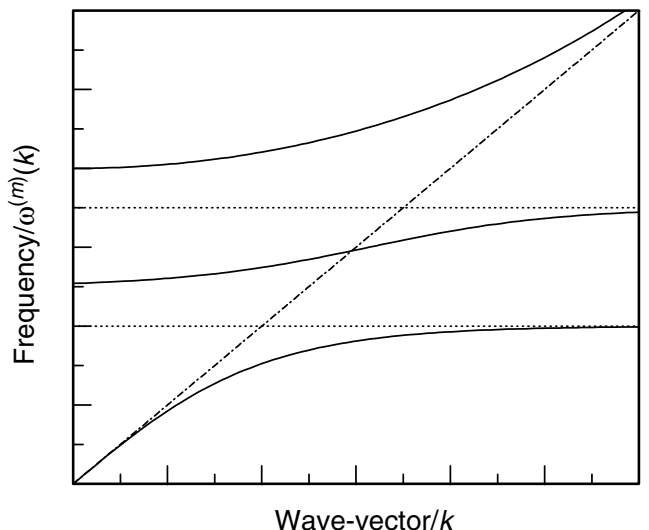

(b)

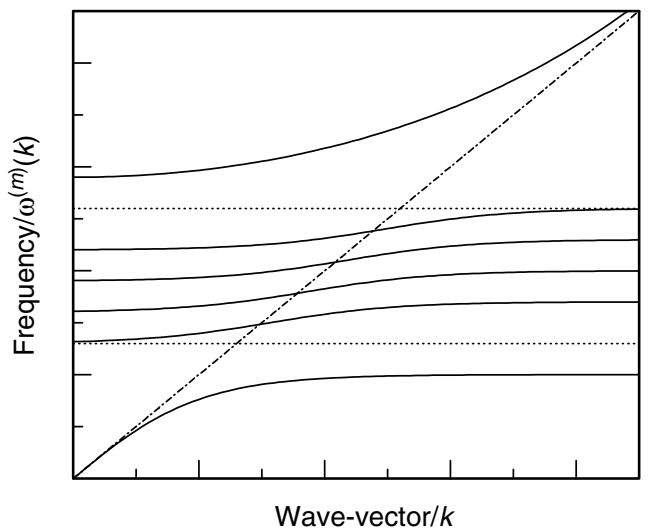

(c)
Figure 1. Schematic curves illustrate the dispersion relationship between the polariton frequency/ $\omega^{(m)}(k)$ and the wave-vector $/ k$. Figure 1(a) illustrates the dispersion if only a single molecular frequency is present (Hopfield model), and (b) the case of two molecular resonances; (c) depicts a situation in which a number of such dispersion branches are present. 
representing a quantum extension of the Lorentz-Lorenz equation. Here $\bar{\alpha}$ is the average molecular polarizability of the homogeneous host, based on an electronically isotropic medium, and $\rho$ is the number density of host molecules.

In the majority of applications to quantum and nonlinear optical phenomena, it is only the photon-like branches of the dispersion curves that are of interest, and the $m$ index in the preceding expressions can generally be left implicit. Again, as we shall be concerned with photonic regions, it is legitimate to engage $a_{\mathbf{k}}^{(\lambda)}$ and $a_{\mathbf{k}}^{\dagger(\lambda)}$ in place of $P_{\mathbf{k}}^{(\lambda)}$ and $P_{\mathbf{k}}^{\dagger(\lambda)}$. In keeping with this policy, we shall from here on once again refer only to photons, although it is understood that the quanta involved are, strictly speaking, optical branch polaritons. The case of vacuum propagation can then be viewed as a special case of the more general formalism. For example, if the refractive index is set to unity in Eq. (19), the group velocity is simply $c$, the polariton operators become identical to those representing the annihilation and creation of pure photons, and the expression reduces directly to the fundamental electric field operator of Eq. (7), multiplied by $\varepsilon_{0}$.

At this juncture we have in place a formalism that fully accounts for the refractive and dissipative modifications of the fundamental fields due to the dispersive electronic properties of the optical medium. This has been achieved not by any phenomenological or other ad hoc approach, but from first principles, using the theoretical methods of molecular QED. As a result, the necessary local field corrections in condensed media naturally emerge from the detailed form of the auxiliary field operators, obviating the need to encompass them indirectly in terms of macroscopic bulk susceptibilities, as is necessary in the semiclassical theory.

\section{PERTURBATIVE DEVELOPMENT}

With the full Hamiltonian given by Eq. (1), the time evolution of the system wavefunction $\psi$ is determined by the time-dependent Schrödinger equation:

$$
i \hbar \frac{\partial \psi(t)}{\partial t}=H \psi(t)
$$

Solutions of high precision, fully incorporating electronic media effects, can be derived on the assumption that the coupling between matter and radiation is treated as a perturbation on the eigenstates of $H_{0}$, where

$$
H_{0}=H_{\mathrm{bath}}+\sum_{\xi}{ }^{\prime} H_{\mathrm{mol}}(\xi)
$$

with

$$
H_{\text {bath }}=H_{\text {rad }}+\sum_{\xi}^{\prime \prime}\left(H_{\text {mol }}(\xi)+H_{\text {int }}(\xi)\right)
$$


In Eq. (25) the prime on the summation denotes its limitation to those molecules whose transitions are engaged either directly or indirectly in the optical response. The double prime on the summation in Eq. (26) denotes the exclusion of those molecules. The eigenstates of $H_{0}$ thus contain products of the eigenstates of the optically prominent molecules and the dressed-photon eigenstates of $H_{\text {bath }}$. As usual, if the system is in an eigenstate of $H_{0}$ at time 0 , the wavefunction at any later time $t$ is expressible as

$$
|\psi(t)\rangle=\exp \left(\frac{-i H_{0} t}{\hbar}\right) U(t, 0)|\psi(0)\rangle
$$

defining a unitary time evolution operator $U(t, 0)$ for the evolution of the system in the time interval $(0, t)$. Substitution of Eq. (27) into Eq. (24) leads to an exact result for $U(t, 0)$ expressible as the following series expansion

$$
U(t, 0)=1+\sum_{m=1}^{\infty}(i \hbar)^{-m} \int_{t_{0}}^{t} \int_{t_{0}}^{t_{1}} \cdots \int_{t_{0}}^{t_{m-1}} \tilde{H}_{\text {int }}\left(t_{1}\right) \tilde{H}_{\text {int }}\left(t_{2}\right) \cdots \tilde{H}_{\text {int }}\left(t_{m}\right) d t_{1} d t_{2} \cdots d t_{m}
$$

where $\tilde{H}_{\text {int }}(t)$ is the interaction representation of the operator responsible for the coupling between light and matter, given by $\tilde{H}_{\text {int }}(t)=\exp \left(i H_{0} t / \hbar\right) H_{\text {int }}$ $\exp \left(-i H_{0} t / \hbar\right)$. In the electric dipole approximation this results in the expression

$$
\tilde{H}_{\text {int }}(t)=-\varepsilon_{0}^{-1} \boldsymbol{\mu} \cdot \tilde{\mathbf{d}}(\mathbf{r}, t)
$$

where the corresponding microscopic electric displacement operator, $\tilde{\mathbf{d}}(\mathbf{r}, t)$ in the interaction representation, may be expressed as a sum of two parts:

$$
\begin{gathered}
\tilde{\mathbf{d}}(\mathbf{r}, t)=\tilde{\mathbf{d}}^{(+)}(\mathbf{r}, t)+\tilde{\mathbf{d}}^{(-)}(\mathbf{r}, t) \\
\tilde{\mathbf{d}}^{(+)}(\mathbf{r}, t)=i \sum_{\mathbf{k}, \lambda, m}\left\{\frac{\hbar v_{g}^{(m)} \omega_{k}^{(m)} \varepsilon_{0}}{2 c V n_{\omega_{k}^{(m)}}}\right\}^{1 / 2}\left(\frac{n_{\omega_{k}^{(m)}}^{2}+2}{3}\right) \mathbf{e}_{\mathbf{k}}^{(\lambda)} a_{\mathbf{k}}^{(\lambda)} \exp \left[i\left(\mathbf{k} \cdot \mathbf{r}-\omega_{k}^{(m)} t\right)\right] \\
\tilde{\mathbf{d}}^{(-)}(\mathbf{r}, t)=-i \sum_{\mathbf{k}, \lambda, m}\left\{\frac{\hbar v_{g}^{(m)} \omega_{k}^{(m)} \varepsilon_{0}}{2 c V n_{\omega_{k}^{(m)}}}\right\}^{1 / 2}\left(\frac{n_{\omega_{k}^{(m)}}^{2}+2}{3}\right) \mathbf{e}_{\mathbf{k}}^{(\lambda)} a_{\mathbf{k}}^{\dagger(\lambda)} \exp \left[-i\left(\mathbf{k} \cdot \mathbf{r}-\omega_{k}^{(m)} t\right)\right]
\end{gathered}
$$


In this form, the time-even and space-odd character of the electric displacement is again apparent. Also, development of the magnetization reveals its time-odd and space-even character.

In developing the quantum amplitude for an optical process, it is necessary to determine the matrix elements of the time evolution operator, and to this end it is frequently expedient to invoke an expansion in terms of operators rather than the embedded time integrals of Eq. (28). The method of resolvent operators, which affords a framework for both perturbative and nonperturbative analysis [28-30], proceeds through the introduction of a retarded Green function, $K_{+}(t)=$ $U(t, 0) \theta(t)$, together with its advanced counterpart, $K_{-}(t)=-U(t, 0) \theta(-t)$, where $\theta(t)$ is the Heaviside function. These functions allow us to extend to infinity the temporal dependence of the evolution operator, enabling us to express the time evolution operator $U_{I}\left(t, t_{0}\right)$ as

$$
U(t, 0)=K_{+}(t)-K_{-}(t)=\frac{1}{2 \pi i} e^{i E_{f} t / \hbar} \int_{-\infty}^{+\infty} e^{i E_{f} t / \hbar}\left[G_{-}(E)-G_{+}(E)\right] d E
$$

where $E_{f}$ denotes the (final) system energy and the retarded and advanced propagators, $G_{+}(E)$ and $G_{-}(E)$, respectively, are Fourier transforms of the retarded and advanced Green functions:

$$
G_{ \pm}(E)=\frac{1}{i \hbar} \int_{-\infty}^{+\infty} e^{i E \tau / \hbar} K_{ \pm}(\tau) d \tau=\lim _{\eta \rightarrow 0+}(E-H \pm i \eta)^{-1}
$$

At this stage it is convenient to define a set of subsystems, each containing one of the optically prominent molecules $\xi$ and the bath. Introducing and expanding in perturbative fashion the corresponding resolvent operator [31]

$$
\begin{aligned}
T_{\operatorname{sub}(\xi)}(z)= & \left(z-H_{0}-H_{\text {int }}(\xi)\right)^{-1} \\
= & \left(z-H_{0}\right)^{-1}+\left(z-H_{0}\right)^{-1} H_{\text {int }}(\xi)\left(z-H_{0}\right)^{-1} \\
& +\left(z-H_{0}\right)^{-1} H_{\text {int }}(\xi)\left(z-H_{0}\right)^{-1} H_{\text {int }}(\xi)\left(z-H_{0}\right)^{-1} \cdots \\
= & \sum_{p=0}^{\infty}\left[T_{0}(z) H_{\text {int }}(\xi)\right]^{p} T_{0}(z)
\end{aligned}
$$

enables the requisite optical amplitude to be determined. Specifically, for a process associated with an initial system state $|i\rangle$ and a final system state $|f\rangle$, we have a quantum probability amplitude that can be evaluated from the equation

$$
\begin{aligned}
c_{f i}=\langle f|U(t, 0)| i\rangle & =\frac{1}{2 \pi i} e^{i E_{f} t / \hbar} \oint e^{-i z t / \hbar}\left\langle f\left|T_{\operatorname{sub}(\xi)}(z)\right| i\right\rangle d z \\
& =\frac{1}{2 \pi i} e^{i E_{f} t / \hbar} \sum_{p=0}^{\infty} \oint e^{-i z t / \hbar}\left\langle f\left|\left[T_{0}(z) H_{\text {int }}(\xi)\right]^{p} T_{0}(z)\right| i\right\rangle d z
\end{aligned}
$$


where the contour for integration encompasses the real axis. Using the method of residues to evaluate the contour integral, and discarding optical frequency oscillatory terms, we thus obtain

$$
c_{f i}=\delta_{f i}-2 \pi i \delta\left(E_{f}-E_{i}\right) \sum_{\xi} M_{f i}(\xi)
$$

The first term denotes the trivial nonprocess in which the initial and final states of the entire system are identical, and can be discarded for any real process. The subsequent term, in which the delta function serves to ensure overall energy conservation, leads to a rate equation expressed by the familiar Golden Rule [32-34]

$$
\Gamma=\left(\frac{2 \pi}{\hbar}\right)\left|\sum_{\xi} M_{f i}(\xi)\right|^{2} \delta\left(E_{f}-E_{i}\right)
$$

cast in terms of a transition matrix whose elements are

$$
\begin{aligned}
M_{f i}(\xi) & =\left\langle f_{\operatorname{sub}(\xi)}\left|H_{\text {int }}(\xi)+H_{\text {int }}(\xi) T_{\operatorname{sub}(\xi)} H_{\text {int }}(\xi)\right| i_{\operatorname{sub}(\xi)}\right\rangle \\
& =\left\langle f_{\operatorname{sub}(\xi)}\left|H_{\text {int }}(\xi)+\sum_{p=0}^{\infty} H_{\text {int }}(\xi)\left[T_{0} H_{\text {int }}(\xi)\right]^{p} T_{0} H_{\text {int }}(\xi)\right| i_{\operatorname{sub}(\xi)}\right\rangle
\end{aligned}
$$

and where the resolvent operators $T_{0}$ and $T_{\operatorname{sub}(\xi)}$ are evaluated for $z=E_{0}$.

\section{TIME ORDERINGS AND STATE SEQUENCES}

For each molecule $\xi$, every other molecule yields a vanishing contribution to the summands in Eq. (39). Hence, by invoking the completeness relation for the subsystem states, the matrix elements $M_{f i}$ can succinctly be expressed as

$$
\begin{aligned}
M_{f i}= & \left\langle f\left|H_{\text {int }}\right| i\right\rangle+\sum_{r^{(1)}} \frac{\left\langle f\left|H_{\text {int }}\right| r^{(1)}\right\rangle\left\langle r^{(1)}\left|H_{\text {int }}\right| i\right\rangle}{\left(E_{i}-E_{r^{(1)}}\right)} \\
& +\sum_{r^{(2)}, r^{(1)}} \frac{\left\langle f\left|H_{\text {int }}\right| r^{(2)}\right\rangle\left\langle r^{(2)}\left|H_{\text {int }}\right| r^{(1)}\right\rangle\left\langle r^{(1)}\left|H_{\text {int }}\right| i\right\rangle}{\left(E_{i}-E_{r^{(2)}}\right)\left(E_{i}-E_{r^{(1)}}\right)} \\
& +\sum_{r^{(3)}, r^{(2)}, r^{(1)}} \frac{\left\langle f\left|H_{\text {int }}\right| r^{(3)}\right\rangle\left\langle r^{(3)}\left|H_{\text {int }}\right| r^{(2)}\right\rangle\left\langle r^{(2)}\left|H_{\text {int }}\right| r^{(1)}\right\rangle\left\langle r^{(1)}\left|H_{\text {int }}\right| i\right\rangle}{\left(E_{i}-E_{r^{(3)}}\right)\left(E_{i}-E_{r^{(2)}}\right)\left(E_{i}-E_{r^{(1)}}\right)}+\cdots
\end{aligned}
$$

where all states and energies are eigenstates of $H_{0}$ and thus relate to the total system containing both the bath and the molecule, with the summations over the 
virtual intermediate states $r^{(1)}, r^{(2)}, \ldots$ taken over all such states excluding $i$ or $f$. In passing it is worth noting that this specifically does not exclude the molecular initial or final state from inclusion in the intermediate state summations, a point that has often been misunderstood but that is quite clear in the QED formulation. The representation of any $m$-photon interaction entails terms involving $m$-photon operators. In view of the linearity in the photon creation and annihilation operators of all the electric and magnetic multipole interactions, this representation generates its leading contribution from the term involving the $m$ th power of $H_{\mathrm{int}}$, and for most processes it is sufficient to consider only the leading nonvanishing term. Writing the system states explicitly as products of molecular and radiation states, we then have

$$
\begin{aligned}
M_{f i}^{(m)}= & \sum_{r_{\mathrm{mol}}^{(1)}} \cdots \sum_{r_{\mathrm{mol}}^{(m-1)}} \sum_{r_{\mathrm{rad}}^{(1)}} \cdots \sum_{r_{\mathrm{rad}}^{(m-1)}}\left\langle f_{\mathrm{rad}} ; f_{\mathrm{mol}}\left|H_{\mathrm{int}}\right| r_{\mathrm{mol}}^{m-1} ; r_{\text {rad }}^{(m-1)}\right\rangle \\
& \times\left\langle r_{\mathrm{rad}}^{(m-1)} ; r_{\mathrm{mol}}^{(m-1)}\left|H_{\mathrm{int}}\right| r_{\mathrm{mol}}^{(m-2)} ; r_{\mathrm{rad}}^{(m-2)}\right\rangle \cdots\left\langle r_{\mathrm{rad}}^{(1)} ; r_{\mathrm{mol}}^{(1)}\left|H_{\mathrm{int}}\right| i_{\mathrm{mol}} ; i_{\mathrm{rad}}\right\rangle \\
& \times\left[\left(E_{i_{\mathrm{mol}}}-E_{r_{\mathrm{mol}}^{(m-1)}}\right)+\left(E_{\mathrm{r}_{\mathrm{rad}}}-E_{\left.r_{\mathrm{rad}}^{(m-1)}\right)}\right)\right]^{-1} \cdots\left[\left(E_{i_{\mathrm{mol}}}-E_{r_{\mathrm{mol}}^{(1)}}\right)\right. \\
& +\left(E_{i_{\mathrm{rad}}}-E_{r_{\mathrm{rad}}^{(1)}}\right]^{-1}
\end{aligned}
$$

In each of the $(m-1)$ summations over the intermediate radiation states $\left|r_{\text {rad }}^{(j)}\right\rangle$, there are only a limited number of possibilities that can make nonvanishing contributions, determined by the sequencing of the creation and annihilation events for the photons emitted and absorbed during the overall interaction. Each of these sequences is conventionally represented using Feynman time-ordered graphs. Calculations based on this method are commonly expedited by the construction of all topologically different diagrams connecting the same initial and final states; the summations over the intermediate states $\left|r_{\text {rad }}^{(1)}\right\rangle$ to $\left|r_{\text {rad }}^{(m-1)}\right\rangle$ in Eq. (41) are then equivalent to summations over the various time orderings. An alternative method based on state sequences [35] enables the complete set of interaction sequences for any process to be cast in the form of a single diagram. The latter method offers a more concise presentation and improved calculational expediency, particularly in the case of high-order interactions.

Optical frequency doubling affords a simple illustration of each of these diagrammatic methods. For clarity, it is expedient to write down the system states for the conversion process (although familiarity with either method enables this information to be read off directly from the diagrams). The initial and final states for the interaction are as follows:

$$
\begin{aligned}
\left|i_{\mathrm{mol}} ; i_{\mathrm{rad}}\right\rangle & =\left|E_{0} ; q(\mathbf{k}, \lambda), q^{\prime}\left(\mathbf{k}^{\prime}, \lambda^{\prime}\right)\right\rangle \\
\left|f_{\mathrm{mol}} ; f_{\mathrm{rad}}\right\rangle & =\left|E_{0} ;(q-2)(\mathbf{k}, \lambda),\left(q^{\prime}+1\right)\left(\mathbf{k}^{\prime}, \lambda^{\prime}\right)\right\rangle
\end{aligned}
$$


Given that the molecule is initially in its ground state, there are initially $q$ photons of the pump mode $(\mathbf{k}, \lambda)$ and $q^{\prime}$ photons of the harmonic mode $\left(\mathbf{k}^{\prime}, \lambda^{\prime}\right)$. There are three possible sequences of photon annihilation and creation $(a, b$, and $c)$ that can provide a route from the initial to the final state, each involving different virtual intermediate states. To avoid confusion, the intermediate state labels $r^{(1)}$ and $r^{(2)}$ are redesignated here as $r$ and $s$, respectively, and the latter appear below with superscripts to identify the route

$$
\begin{aligned}
& \left.\begin{array}{l}
\left|r_{\mathrm{mol}} ; r_{\mathrm{rad}}^{a}\right\rangle=\left|E_{r} ;(q-1)(\mathbf{k}, \lambda), q^{\prime}\left(\mathbf{k}^{\prime}, \lambda^{\prime}\right)\right\rangle \\
\left|s_{\mathrm{mol}} ; s_{\mathrm{rad}}^{a}\right\rangle=\left|E_{s} ;(q-2)(\mathbf{k}, \lambda), q^{\prime}\left(\mathbf{k}^{\prime}, \lambda^{\prime}\right)\right\rangle
\end{array}\right\} \\
& \left.\begin{array}{l}
\left|r_{\mathrm{mol}} ; r_{\mathrm{rad}}^{b}\right\rangle=\left|E_{r} ;(q-1)(\mathbf{k}, \lambda), q^{\prime}\left(\mathbf{k}^{\prime}, \lambda^{\prime}\right)\right\rangle \\
\left|s_{\mathrm{mol}} ; s_{\text {rad }}^{b}\right\rangle=\left|E_{s} ;(q-1)(\mathbf{k}, \lambda),\left(q^{\prime}+1\right)\left(\mathbf{k}^{\prime}, \lambda^{\prime}\right)\right\rangle
\end{array}\right\} \\
& \left.\begin{array}{l}
\left|r_{\mathrm{mol}} ; r_{\mathrm{rad}}^{c}\right\rangle=\left|E_{r} ; q(\mathbf{k}, \lambda),\left(q^{\prime}+1\right)\left(\mathbf{k}^{\prime}, \lambda^{\prime}\right)\right\rangle \\
\left|s_{\mathrm{mol}} ; s_{\mathrm{rad}}^{c}\right\rangle=\left|E_{s} ;(q-1)(\mathbf{k}, \lambda),\left(q^{\prime}+1\right)\left(\mathbf{k}^{\prime}, \lambda^{\prime}\right)\right\rangle
\end{array}\right\}
\end{aligned}
$$

as represented by the three time-ordered diagrams of Fig. 2. For example, in Fig. 2a, the sequence of interactions is as follows. First, a photon of the pump mode is annihilated by a molecule in its ground state $|0\rangle$, which thereby undergoes a transition to a state $|r\rangle$. A second pump photon is then annihilated, and the molecule proceeds to a state $|s\rangle$. Finally, a harmonic frequency photon is emitted, and the molecule returns to its ground state. Figures $2 \mathrm{~b}$ and $2 \mathrm{c}$ represent the two other possible sequences in which emission of the harmonic photon precedes either one, or both of the pump photon interactions. It is important to emphasize that no single time-ordered diagram represents a physically distinguishable process; these diagrams are ultimately only calculational aids based on

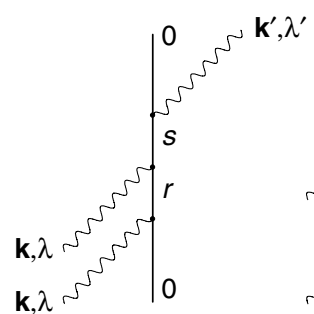

(a)

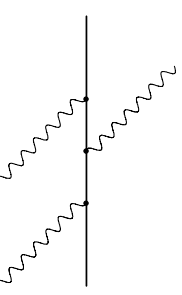

(b)

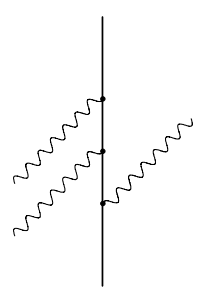

(c)

Figure 2. The three time-ordered diagrams representing second harmonic generation. Pump photons of wave vector $\mathbf{k}$ and polarization $\lambda$ impinge on the molecule from the left and the subsequent harmonic $\left(\mathbf{k}^{\prime}, \lambda^{\prime}\right)$ leaves the molecular world line from the right. We assume the initial and final state of the molecule is the ground state 0 ; the intermediate states are labelled $r$ and $s$. 


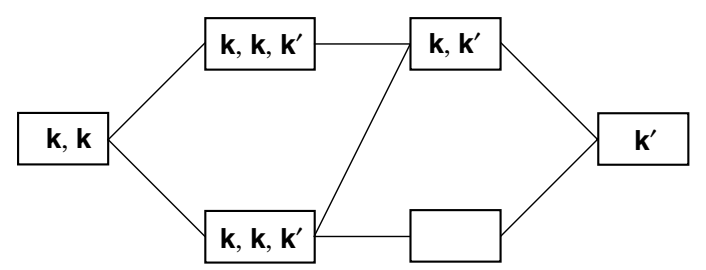

Figure 3. State sequence diagram for SHG. The intial state is represented by the solitary box in the column on the left and the final state by the corresponding the box on the right; columns with more then one box indicate virtual states $r$ and $s$. An example is afforded by the uppermost route through the diagram, which corresponds identically to the time-ordering of Fig. 2(c).

the approximations of perturbation theory. Collectively, the photon creation and annihilation events that take place at each molecule appear simultaneously, as far as real experimental measurements with finite time resolution are concerned. However, the time-energy uncertainty relation does permit short-lived intermediate states that are not properly energy-conserving. This helps explain why it is necessary to include the diagrams corresponding to extremely counterintuitive time sequences such as that of Fig. 2c. Nonetheless, all possible interaction sequences must be included in calculating any matrix element.

The state sequence diagram of Fig. 3 accommodates all three routes between the initial and final states; those states are represented by boxes on the far left and far right of the diagram, respectively. The intervening columns represent intermediate system states $r$ and $s$ connected by links that represent valid operations by $H_{\text {int }}$ on a preceding state. In these diagrams, lines thus represent interactions and vertices (where data boxes appear) represent states; in this sense they bear a reciprocal space relationship to the Feynman diagrams, where the converse applies.

\section{TENSOR REPRESENTATION}

To proceed with the general development, it is useful to extract from the quantum amplitude those elements that involve properties belonging solely to the radiation and molecular tensors, respectively. Thus we formulate matrix elements through the appropriate tensor products for deployment in the Golden Rule.

Consider an $m$-photon process involving modes of radiation $\left(\mathbf{k}_{m}, \lambda_{m}\right), \ldots$, $\left(\mathbf{k}_{1}, \lambda_{1}\right)$, all potentially different, initially containing $q_{m}, \ldots, q_{1}$ photons, respectively. Equation (41) may be factorized as a tensor product of two terms, only one of which is dependent on any intrinsic molecular properties:

$$
\begin{aligned}
M_{f i}\left\{\left(\mathbf{k}_{1}, \lambda_{1}\right) \cdots\left(\mathbf{k}_{m}, \lambda_{m}\right)\right\}= & (-1)^{m} \varepsilon_{0}^{-m} \alpha_{i_{m} \cdots i_{2} i_{1}}\left(-\omega_{m} ; \omega_{1}, \ldots, \omega_{n}\right) \\
& \times \rho_{i_{m} \cdots i_{2} i_{1}}\left\{\left(\mathbf{k}_{m}, \lambda_{m}\right), \ldots,\left(\mathbf{k}_{1}, \lambda_{1}\right)\right\}
\end{aligned}
$$


Specifically, this equation entails the $m$-fold tensor contraction of $\boldsymbol{\alpha}^{(m)}\left(\omega_{m}, \ldots\right.$, $\left.\omega_{1}\right)$, a rank- $m$ microscopic nonlinear polarisability tensor containing all the molecular variables (with a parametric dependence on the optical frequencies), with $\boldsymbol{\rho}^{(m)}\left\{\left(\mathbf{k}_{m}, \lambda_{m}\right), \ldots,\left(\mathbf{k}_{1}, \lambda_{1}\right)\right\}$, a tensor constructed solely from radiation parameters, including all the necessary local field corrections. Note the incorporation in Eq. (47) of a sign prefactor, serving to redefine each energy denominator so that the absorption of a photon is associated with a negative radiative frequency and emission with a positive frequency. This results in nonlinear polarisability expressions conforming to the usual conventions. An example might be $n$-harmonic generation where a tensor written as $\boldsymbol{\alpha}^{(m)}\left(-\omega_{m}\right.$; $\left.\omega_{1}, \ldots, \omega_{1}\right)$ indicates the absorption $n$ photons of frequency $\omega_{1}$ and emission of a single photon at a frequency $\omega_{m} \equiv n \omega_{1}$.

We postpone to a later section a detailed explanation of the explicit expressions for the molecular tensors $\boldsymbol{\alpha}^{(m)}$; we next identify the structure of the radiation tensor $\boldsymbol{\rho}^{(m)}$, which in the electric dipole approximation is given by the following:

$$
\begin{aligned}
\boldsymbol{\rho}^{(m)}\left\{\left(\mathbf{k}_{m}, \lambda_{m}\right), \ldots,\left(\mathbf{k}_{1}, \lambda_{1}\right)\right\}= & \left\langle\left(m_{m}+1\right)(\mathbf{k}, \lambda)\left|\mathbf{d}^{\perp}\right|\left(m_{m}\right)(\mathbf{k}, \lambda)\right\rangle, \ldots, \\
& \left\langle\left(m_{1}-1\right)(\mathbf{k}, \lambda)\left|\mathbf{d}^{\perp}\right|\left(m_{1}\right)(\mathbf{k}, \lambda)\right\rangle
\end{aligned}
$$

In general, to incorporate the matrix elements of Eq. (47) into the rate equation (38), it is necessary to sum, over all molecules in the system, the tensor product entailed in the former-and to this end it proves useful to isolate the one part of the above radiation tensor that is molecule-specific. This simply reflects the fact that the tensor is a field quantity, sensitive to the position of the molecule at which it is evaluated, as follows from the phase factors in (19) and (20). The tensor representing the radiation field for the interaction at molecule $\xi$ may, in fact, be written in the following general form, irrespective of the order or nature of the multipolar interactions involved:

$$
\boldsymbol{\rho}(\xi)=\boldsymbol{\rho}^{\prime} \exp \left(i \Delta \mathbf{k} \cdot \mathbf{R}_{\xi}\right)
$$

Here $\rho^{\prime}$ is a position-independent radiation tensor, $\mathbf{R}_{\xi}$ is the position vector of the molecule relative to an arbitrary fixed origin, and $\Delta \mathbf{k}$ the wavevector mismatch for the process as defined by

$$
\Delta \mathbf{k}=\sum_{r}^{m} g_{r} \mathbf{k}_{r}
$$

where $g_{r}$ assumes the value of +1 for each absorbed photon and -1 for each emitted photon. For instance, the radiation tensor for frequency doubling carries the phase factor $\exp \left[i\left(2 \mathbf{k}-\mathbf{k}^{\prime}\right) \cdot \mathbf{R}_{\xi}\right]$, and hence $\Delta \mathbf{k}=\left(2 \mathbf{k}-\mathbf{k}^{\prime}\right)$. Such features 
are responsible for the all-important issue of coherence. Combining the above results, it thus transpires that any optical process rate may be written in a general Golden Rule form as

$$
\Gamma=\frac{2 \pi}{\hbar}\left|\sum_{\xi}^{N} \varepsilon_{0}^{-m} \alpha_{i_{m} \cdots i_{2} i_{1}}^{(\xi)} \rho_{i_{m} \cdots i_{2} i_{1}}^{\prime} \exp \left(i \Delta \mathbf{k} \cdot \mathbf{R}_{\xi}\right)\right|^{2} \delta\left(E_{i}-E_{f}\right)
$$

This equation lies at the heart of the theoretical development; the precise structure of the molecular and radiation tensors it involves will depend on the detailed nature of the optical interaction to be modeled.

\section{CONSTRUCTION OF RADIATION TENSORS}

Having stated that all optical interactions can be modeled through Eq. (51), we now outline the explicit details of both radiation and molecular tensors embedded in the matrix element. As we have shown, the radiative features that are of interest are cast in the tensor $\rho$ introduced in Eq. (48). For any optical process the components of this tensor are explicitly given by a positionindependent expression, which follows from Eq. (49):

$$
\begin{aligned}
\rho^{\prime}\left\{\left(\mathbf{k}_{m}, \lambda_{m}\right), \ldots,\left(\mathbf{k}_{1}, \lambda_{1}\right)\right\}= & \rho_{i_{m} \cdots i_{1}}^{\prime}\left\{\left(\mathbf{k}_{m}, \lambda_{m}\right), \ldots,\left(\mathbf{k}_{1}, \lambda_{1}\right)\right\} \\
\rho_{i_{m} \cdots i_{1}}^{\prime}\left\{\left(\mathbf{k}_{m}, \lambda_{m}\right), \ldots,\left(\mathbf{k}_{1}, \lambda_{1}\right)\right\}= & \left\{-i^{(m)}\right\} s_{i_{m} \cdots i_{1}}\left\{\left(\mathbf{k}_{m}, \lambda_{m}\right), \ldots,\left(\mathbf{k}_{1}, \lambda_{1}\right)\right\} \\
& \times \prod_{i=1}^{m}\left[\left(\frac{\hbar v_{g}^{(m)} \omega_{k}^{(m)} \varepsilon_{0}}{2 c V n_{\omega_{k}}}\right)^{1 / 2}\left(\frac{n_{\omega_{k}}^{2}+2}{3}\right) \sqrt{q}\right]_{\left(\left|\mathbf{k}_{i}\right|\right)}
\end{aligned}
$$

Here, $q_{i}$ is the number of photons in the $i$ th mode within the quantization volume $V$. If no photons of a particular mode are initially present, as would be the case for spontaneous emission processes, $q_{i}$ is equal to unity, as the photon creation operator then acts on the vacuum state. The symbol $\mathbf{s}\left\{\left(\mathbf{k}_{m}, \lambda_{m}\right), \ldots,\left(\mathbf{k}_{1}, \lambda_{1}\right)\right\}$ represents a polarization tensor defined by

$$
S_{i_{m} \cdots i_{1}}\left\{\left(\mathbf{k}_{m}, \lambda_{m}\right), \ldots,\left(\mathbf{k}_{1}, \lambda_{1}\right)\right\}=e_{i_{1}}\left(\mathbf{k}_{1}, \lambda_{1}\right) \cdots e_{i_{m}}\left(\mathbf{k}_{m}, \lambda_{m}\right) \equiv e_{i_{1}} \cdots e_{i_{m}}
$$

The arguments associated with each unit vector are now dropped for brevity. The polarization unit vectors $\mathbf{e}_{i}$ refer to each photon involved in the interaction process. The polarization vectors are represented as above for each photon that is annihilated, but created photons carry the overbar to represent complex 
conjugation, thus allowing for the possibility that the light is circularly or elliptically polarised. It is also customary if two photons are from the same mode, as for example would be the case in single-color two-photon absorption or a harmonic process, to place parentheses around the appropriate indices to signify permutational symmetry.

In order to relate to the specific conditions produced by a given laser source, it is clearly necessary to express results in terms of physically meaningful radiation parameters in lieu of the artificial quantization volume $V$ and photon number $q$ that appear in Eq. (52). The procedure for this reformulation allows consideration of pump radiation states characterized by various forms of photon statistics, leading to results appropriate for several different kinds of intensity distribution. In an earlier review [1] it was shown how to develop theory in terms of quantum optical states more realistic than the zero-fluctuation number states $|q(\mathbf{k}, \lambda)\rangle$ hitherto employed in the general formulation. Although these states are the most usual basis for QED calculations based on time-dependent perturbation theory, they are associated with infinite phase uncertainty and do not adequately represent any real laser input. One basis set of states that appears rather better suited to the modeling of laser radiation is the overcomplete set represented by the coherent states $|\beta(\mathbf{k}, \lambda)\rangle$. These states, characterized for any given radiation mode by minimization of the uncertainty in phase and occupation number [36,37], are eigenstates of the corresponding annihilation operators, satisfying the result

$$
a^{(\lambda)}(\mathbf{k})|\beta(\mathbf{k}, \lambda)\rangle=\beta(\mathbf{k}, \lambda)|\beta(\mathbf{k}, \lambda)\rangle
$$

where $\beta(\mathbf{k}, \lambda)$ is a complex number whose modulus relates to the mean photon number $q$ through $q=|\beta(\mathbf{k}, \lambda)|^{2}$. It should nonetheless be mentioned that the employment of coherent or other states can, if caution is not exercised, produce spurious features resulting from the fact that they are not eigenstates of the radiation Hamiltonian, so that neither photon creation followed by annihilation nor annihilation followed by creation is an identity operation. This feature is commonly overlooked, but it provides one of the best reasons for working with number states if quantum optical aspects are not at issue.

Rate equations expressed in terms of mean photon number and quantization volume are still not directly applicable to experiment. Moreover, since the quantization volume is no more than a theoretical artifact, it must invariably cancel out in any final result. However, the ratio of these two quantities, which represents a mean photon density, is directly related to the mean irradiance, and the relationship may be derived as follows. Consider a quantization volume represented by a small cube of space of side length $l$ and volume $V$ through which the incident beam passes; by definition, this cube contains on average $q$ photons of circular frequency $\omega_{k}$, and its energy content is $q \hbar \omega_{k}$ (see Fig. 4). For 


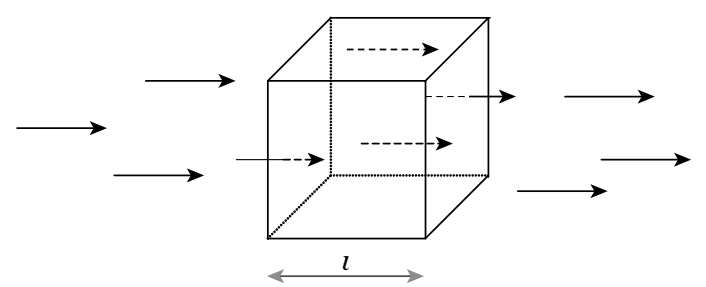

Figure 4. The schematic illustrates a photon flux through a quantization volume. Each side of the chosen cube is assigned a length $l$. The photons traverse the box in a time $l / v_{g}$ where $v_{g}$ is the group velocity in the medium.

a group of $q$ photons with a mean group velocity $v_{g}$, it takes a time $l / v_{g}$ for the energy $q \hbar \omega_{k}$ to traverse the cube; hence the mean irradiance $I\left(\omega_{k}\right)$ (power per unit beam cross-sectional area) is given by $\left\{q \hbar \omega_{k} /\left(l / v_{g}\right)\right\} / l^{2}$, so that

$$
I\left(\omega_{k}\right)=\frac{q \hbar v_{g} \omega_{k}}{V}
$$

In passing we may note that the mean interval $\tau$ between photon arrival times for any one molecule of physical cross section $\sigma$ is directly related to $I\left(\omega_{k}\right)$ through

$$
\tau=\frac{\hbar \omega_{k}}{I\left(\omega_{k}\right) \sigma}
$$

The parameter $\tau$ is rarely featured in the literature but in the case of nonparametric excitation and decay processes in molecular media, its value relative to the decay lifetime affords a useful gauge of excitation efficiency.

For the generation of radiation through any incoherent optical process, the general freedom in propagation direction means that each photon is spontaneously created into any one of an infinite set of radiation states, subject to energy conservation. For a collection of free molecules, uncertainty in the molecular state energy, due to the presence of densely packed quasicontinuous vibrational and rotational energy levels, ensures that a density of states representation can legitimately replace the delta function of Eq. (51); see, for example, the work by Craig and Thirunamachandran [15].

Even in coherent processes whose nature serves to define the principal direction of the emergent radiation, and where the initial and final molecular states are necessarily identical, the general theory leads to quantum amplitudes in which the final state of the radiation field is not yet completely specified with regard to the wavevector and polarization of the emitted radiation. As such, the sums over all possible values of $\mathbf{k}^{\prime}$ and $\lambda^{\prime}$ should remain in the general amplitudes of the radiation tensor $\boldsymbol{\rho}^{\prime}$. However, the restrictions imposed on parametric 
processes by virtue of energy conservation and wavevector matching conditions [1] ensures that radiation is emitted into a small pencil of solid angle centered around $\mathbf{k}^{\prime}+d \mathbf{k}^{\prime}$, where the prime indicates that the photon is emitted. In effecting the associated sums, we achieve a result of a form that correctly loses dependence on the quantization volume (i.e., except for processes occurring in geometrically confined microcavities where the quantization volume retains physical significance). In the limit of a large quantization volume, the sum over $\mathbf{k}^{\prime}$ is conveniently replaced by an integral of the form

$$
\frac{1}{V} \sum_{\mathbf{k}^{\prime}} \underset{V \rightarrow \infty}{\Longrightarrow} \int \frac{d^{3} \mathbf{k}^{\prime}}{(2 \pi)^{3}}=\frac{1}{(2 \pi)^{3}} \int_{0}^{\infty} \oint k^{\prime 2} d k^{\prime} d \Omega
$$

where it is understood that the solid angle $d \Omega$ extends over all angles. For coherent emission into a pencil of solid angle $d \Omega(\ll 4 \pi$ steradians) centered around $\mathbf{k}^{\prime}$, it is legitimate to substitute for the sum over $\mathbf{k}^{\prime}$ by

$$
\sum_{\mathbf{k}^{\prime}} \Longrightarrow \frac{d \Omega V}{(w \pi)^{3}} \int_{0}^{\infty} k^{\prime 2} d k^{\prime}
$$

Using Eq. (58), any remaining $V$ factor can be successfully removed from the appropriate expressions.

To illustrate a case to be revisited in detail later, we explicitly derive the rate for coherent second-harmonic generation in a system containing $M$ molecules. Using the general expression Eq. (52) for the radiation tensor together with the Golden Rule, and retaining a sum over the emitted harmonic in the matrix elements, we first obtain an expression of the form

$$
\begin{aligned}
\Gamma_{\mathrm{coh}}= & \frac{2 \pi}{\hbar \varepsilon_{0}^{6}}\left(\frac{\hbar v_{g} \omega_{k} \varepsilon_{0}}{2 c V n_{\omega_{k}}}\right)^{2}\left(\frac{n_{\omega_{k}}^{2}+2}{3}\right)^{4} q(q-1) \\
& \times \sum_{\mathbf{k}^{\prime}, \lambda^{\prime}}\left(\eta_{M}-M\right)\left(\frac{\hbar v_{g}^{\prime} \omega_{k^{\prime}}^{\prime} \varepsilon_{0}}{2 c V n_{\omega_{k^{\prime}}}}\right)\left(\frac{n_{\omega_{k^{\prime}}^{\prime}}^{2}+2}{3}\right)^{2}\left|\beta_{i(j k)}(-2 \omega ; \omega, \omega) \bar{e}_{i}^{\prime} e_{j} e_{k}\right|^{2} \\
& \times \delta\left(\hbar \omega_{k}-2 \hbar \omega_{k}\right)
\end{aligned}
$$

Several points deserve mention. First, retention of the sum over the harmonic wavevector and polarization signifies an initial condition that assumes the absence of any harmonic radiation prior to interaction, so that no direction is favored on the basis of stimulated emission (i.e., although the emergent wavevectors are equal in magnitude, they may differ in direction). Secondly, although photon branch indices are suppressed for conciseness, they are to be 
regarded as still implicit. Finally, the parentheses around the molecular tensor indices represent index symmetry. (This will be explained in more detail when we deal with the molecular tensors explicitly in the following sections.)

The delta function in Eq. (59) serves to ensure energy conservation, while the factor $\left(\eta_{M}-M\right)$ signifies the extent to which photon momentum is conserved, as determined by wavevector matching. As shown previously $[1,38]$, we have $\eta_{M}=M^{2}$ in the limit of exact wavevector matching. Now, substituting Eqs. (55) and (58) into (59) allows the rate of coherent harmonic production, into an infinitesimal solid angle $d \Omega$, to be expressed as follows:

$$
\begin{aligned}
d \Gamma_{\mathrm{coh}} & =\frac{I^{2}\left(\omega_{\mathbf{k}}\right) g_{\omega_{k}}^{(2)} d \Omega}{4 \hbar(2 \pi)^{2} c^{2} \varepsilon_{0}^{3} n_{\omega_{k}}^{2}}\left(\frac{n_{\omega_{k}}^{2}+2}{3}\right)^{4} \sum_{\lambda^{\prime}} \int_{0}^{\infty} k^{\prime 2} d k^{\prime} \frac{d \omega_{k^{\prime}}^{\prime}}{d k^{\prime}}\left(\frac{\hbar \omega_{1}^{\prime}}{2 c n_{\omega_{k^{\prime}}^{\prime}}}\right) \\
& \times\left(\frac{n_{\omega_{k^{\prime}}^{\prime}}^{2}+2}{3}\right)^{2}\left|\beta_{i(j k)}(-2 \omega ; \omega, \omega) \bar{e}_{i}^{\prime} e_{j} e_{k}\right|^{2} \delta\left(\hbar \omega_{k^{\prime}}^{\prime}-2 \hbar \omega_{k}\right)\left(\eta_{M}-M\right)
\end{aligned}
$$

Here the group velocity of the harmonic wave is written explicitly as $d \omega_{k^{\prime}}^{\prime} / d k^{\prime}$, and we have introduced the second-order degree of coherence $g^{(2)}$ as appropriate for a generalization beyond number states

$$
g^{(2)}=\frac{\langle q(q-1)\rangle}{\langle q\rangle^{2}}
$$

where the angular brackets indicate expectation values based on the number state operators, $q \equiv a^{\dagger} a$. We proceed with the assumption that the output is polarized, thus obviating the need for the polarization sum $\lambda^{\prime}$. Then, using the definition $k^{\prime}=\omega_{k^{\prime}}^{\prime} n_{\omega_{k^{\prime}}^{\prime}} / c$ and exploiting delta function properties and after a little algebra, we finally arrive at the expression

$$
\begin{aligned}
d \Gamma_{\mathrm{coh}}= & \frac{I^{2}\left(\omega_{k}\right) g_{\omega_{k}}^{(2)} d \Omega \omega_{k}^{3} n_{2 \omega_{k}}}{4 \hbar c^{5} \pi^{2} \varepsilon_{0}^{3} n_{\omega_{k}}^{2}}\left(\frac{n_{\omega_{k}}^{2}+2}{3}\right)^{4}\left(\frac{n_{2 \omega_{k}}^{2}+2}{3}\right)^{2} \\
& \times\left|\beta_{i(j k)}(-2 \omega ; \omega, \omega) \bar{e}_{i}^{\prime} e_{j} e_{k}\right|^{2}\left(\eta_{M}-M\right)
\end{aligned}
$$

which is best recast in terms of a radiant intensity of harmonic emission by using

$$
I\left(\mathbf{k}^{\prime}\right)=\left(\hbar \omega_{k^{\prime}}^{\prime}\right) \frac{d \Gamma}{d \Omega}=\left(2 \hbar \omega_{k}\right) \frac{d \Gamma}{d \Omega}
$$

Significantly, the results of Eqs. (62) and (63) closely resemble those calculated using the more familiar vacuum electric field operators, modified by the 
inclusion of refractive index-dependent correction factors (see, e.g., Refs. 1 and 15). This reflects the fact that the rates calculated for any interaction using those methods can, in some sense, incorporate the necessary media contributions if the matrix elements are phenomenologically modified by the factors $n_{\omega_{k}}^{-1 / 2}\left\{\left(n_{\omega_{k}}^{2}+2\right) / 3\right\}$ or $n_{\omega_{k^{\prime}}^{\prime}}^{1 / 2}\left\{\left(n_{\omega_{k^{\prime}}^{\prime}}^{2}+2\right) / 3\right\}$, for each absorbed or emitted photon, respectively, where $n_{\omega_{k}}$ is the frequency-dependent refractive index. This is a simple prescription that lacks the details of the underlying physics, including the implicit photonic branch indexing. The advantage of the complete method described in the course of this review is that it is directly amenable to systems of significantly greater optical complexity. In contrast to developments that directly dress the molecular response with the local field factors, casting the molecular optical response tensor as a microscopic representative of a bulk susceptibility, Eq. (62) explicitly retains all such factors whose value is determined by the properties of the input radiation, as modified within the bulk of the nonlinear medium.

\section{PUMP PHOTONICS}

Whilst the above is perfectly adequate for the description of processes observed with continuous-wave (cw) input, proper representation of the optical response to pulsed laser radiation requires one further modification to the theory. It is commonly thought difficult to represent pulses of light using quantum field theory; indeed, it is impossible if a number state basis is employed. However by expressing the radiation as a product of coherent states with a definite phase relationship, it is relatively simple to construct a wavepacket to model pulsed laser radiation [39]. The physical basis for this approach is that pulses necessarily have a finite linewidth and therefore in fact entail a large number of radiation modes, so that for the pump radiation, it is appropriate to construct a coherent superposition

$$
\left|i_{\mathrm{rad}}\right\rangle=\prod_{l}\left|\alpha\left(\omega_{l}\right)\right\rangle
$$

and where

$$
\left|\alpha\left(\omega_{l}\right)\right|=q_{l}^{1 / 2}
$$

represents the mean number of photons in the mode labeled by the (positive or negative) integer $l$. For simplicity, it may be assumed that each mode is associated with the same direction of propagation and polarization, so that the frequency label uniquely identifies each component. If the central frequency is $\omega_{0}$ and the interval between adjacent modes is $\Omega$, then we can write

$$
\omega_{l}=\omega_{0}+l \Omega
$$


which, with $\Omega=\pi v_{g}\left(\omega_{0}\right) / L\left[v_{g}\left(\omega_{0}\right)\right.$ denoting the intracavity speed of light at frequency $\omega_{0}$ ], serves to represent the frequency distribution of a laser with optical cavity length $L$. A phase relationship between the axial cavity modes, corresponding to perfect mode locking, can now be enforced by writing

$$
\alpha\left(\omega_{l}\right)=q_{l}^{1 / 2} \exp \left[-i\left(\omega_{l} \tau\right)\right]+\varphi
$$

with a suitable value for $\tau$ and arbitrary $\varphi$. When the initial state defined by (64) is made subject to this condition and employed in the calculation of matrix elements as in Eq. (41), this leads to the representation of a pulse train described by the following temporal envelope function $J(t)$ [39]:

$$
J(t)=\sum_{l}\left(q_{l} \omega_{l}\right)^{1 / 2} \exp [-i l \Omega(t+\tau)]
$$

where the time $t$ arises through evaluation of the matrix elements of $\tilde{\mathbf{d}}^{(+)}$as given by Eq. (31). Choosing $\tau=-\pi / \Omega$ places time zero exactly in between two successive pulses, such that $J(0) \approx 0$ and the interaction is smoothly switched on. By extension of these principles to a continuous frequency distribution, single pulses of radiation can be entertained in the theory through the envelope function

$$
J(t)=\int A(\omega) \exp [-i \omega(t+\tau)] d t
$$

The net result of incorporating all these modifications in the theory of harmonic emission, or any other process entailing the annihilation of $n$ photons from the pump radiation, is that we now have the following prescription:

$$
\left[\frac{q !}{(q-n) !}\right] \rightarrow J^{2 n} \omega^{-n}
$$

For coherent state light, each and every coherence factor takes the value of unity, and it may be observed that the result of effecting Eq. (70) is that a timedependent irradiance $I_{\omega}(t)$ now appears, properly defined through

$$
I_{\omega}(t)=\left(\frac{\hbar c}{V}\right) J^{2}(t)
$$

To complete the reformulation of results in terms of physically meaningful parameters, and to relax the unduly restrictive assumption of the last section, we now consider the possibility of stimulated emission for photons generated by the 
optical process of interest, as, for example, in the case of strong harmonic pumping. This leads to a matrix element containing a factor $\left(q^{\prime}+1\right)^{1 / 2} / V^{1 / 2}$, indicating that the rate becomes linearly dependent on $\left(q^{\prime}+1\right) / V$. When $q^{\prime}$ is large, the rate is essentially proportional to the harmonic photon density. In the light of the preceding remarks on the pump radiation, it is nonetheless for many reasons inadvisable to work in terms of $q^{\prime}$. Number states are hardly appropriate, nor is it sensible to suppose that all harmonic photons are delivered into a single radiation mode. Under conditions of strong emission pumping, it is better to gauge the mean number of $n$-harmonic photons by employment of the relation

$$
q^{\prime}=n^{-1}\left(q_{0}-q\right)
$$

where $q_{0}$ is the initial number of pump photons. Equation (72), which basically reflects energy conservation, may be regarded as an integrated form of the generalised Manley-Rowe relation

$$
\frac{d\left\{I\left(\omega_{\mathbf{k}}\right) / \omega_{\mathbf{k}}\right\}}{d z}=\frac{-q d\left\{I\left(\omega_{\mathbf{k}^{\prime}}^{\prime}\right) / \omega_{\mathbf{k}^{\prime}}^{\prime}\right\}}{d z}
$$

[40]. The $q^{\prime}$ that appears in the rate equations is best interpreted as a ratio of the stimulated to the spontaneous emission rate (see, e.g., Ref. 41).

\section{CONSTRUCTION OF MOLECULAR RESPONSE TENSORS}

In this section we address the detailed form, and in particular the dispersion behavior, of the molecular response tensors. We note that the frequency dependence of nonlinear polarisabilities and their sum rules have been the subject of a series of incisive works by Bishop and others; see, for example, the paper by Bishop and DeKee [42]. In addressing dispersion behavior below, we follow the same general principles, but at the outset we invoke excited-state damping to allow for the incorporation of lineshape. This is a matter that, once its context is established below, we shall return to in the following section.

To begin, from Eqs. (41) and (47) the explicit result for the nonlinear polarizability $\boldsymbol{\alpha}^{(m)}$ that mediates an $m$-photon process may be written, in the electric dipole approximation, as follows:

$$
\begin{aligned}
\boldsymbol{\alpha}^{(m)}= & \sum_{r_{\mathrm{mol}}^{(1)}} \cdots \sum_{r_{\mathrm{mol}}^{(m-1)}} \sum_{r_{\mathrm{rad}}^{(1)}} \cdots \sum_{r_{\mathrm{rad}}^{(m-1)}}\left\langle f_{\mathrm{mol}}|\boldsymbol{\mu}| r_{\mathrm{mol}}^{(m-1)}\right\rangle\left\langle r_{\mathrm{mol}}^{(m-1)}|\boldsymbol{\mu}| r_{\mathrm{mol}}^{(m-2)}\right\rangle \cdots\left\langle r_{\mathrm{mol}}^{(1)}|\boldsymbol{\mu}| i_{\mathrm{mol}}\right\rangle \\
& {\left[\left(\tilde{E}_{r_{\mathrm{mol}}^{(m-1)}}-\tilde{E}_{i_{\mathrm{mol}}}\right)+\left(E_{r_{\mathrm{rad}}^{(m-1)}}-E_{i_{\mathrm{rad}}}\right)\right]^{-1} \cdots\left[\left(\tilde{E}_{r_{\mathrm{mol}}^{(1)}}-\tilde{E}_{i_{\mathrm{mol}}}\right)+\left(E_{r_{\mathrm{rad}}^{(1)}}-E_{i_{\mathrm{rad}}}\right)\right]^{-1} }
\end{aligned}
$$


The summations over all possible intermediate radiation states are accommodated by reference to the various contributing time orderings, or pathways through state-sequence diagrams, and generally result in a set of terms. Each term has a different frequency dependence, as determined by the structure of its energy denominator. The tildas appearing over the molecular energies in Eq. (74) represent a complex representation that includes the effects of damping, to be discussed in detail below. In general, all molecular states carry such damping; only in the special case of the lowest energy (ground) state is the damping redundant. When written with explicit reference to its frequency arguments, the ordering of tensor subscripts in $\boldsymbol{\alpha}^{(m)}$ is assumed to relate identically to the ordering of the frequencies. Thus, for example, in writing the component $\alpha_{i j k}^{(3)}\left(-\omega_{3} ; \omega_{2}, \omega_{1}\right)$ of the nonlinear optical tensor that mediates sum-frequency conversion, the index $i$ corresponds to the $\omega_{3}$ photon interaction, $j$ to the $\omega_{2}$, and $k$ to the $\omega_{1}$ interaction. Since molecular response tensors are seldom completely index-symmetric [43], preserving an unambiguous correlation between indices and photon frequencies is a very necessary consideration. In the time-ordered diagrams, each interaction vertex carries the same index for the corresponding photon in each diagram, so that the subscript ordering on the molecular interaction vertices varies from diagram to diagram. On state-sequence diagrams, the same index set labels the interaction lines denoting state connections.

It is instructive to take as a first example the general expression for molecular polarizability, the response tensor that formally mediates elastic light scattering in the electric dipole approximation. The result is obtained by application of Eq. (74) with $m=2$ (one photon is annihilated and another of the same frequency is created). Here there are only two time orderings, or state-sequence pathways, as illustrated in Figs. 5 and 6, respectively. Each generates a term whose numerator is a product of transition dipole moment components. For

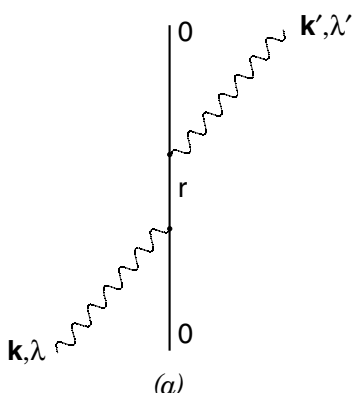

(a)

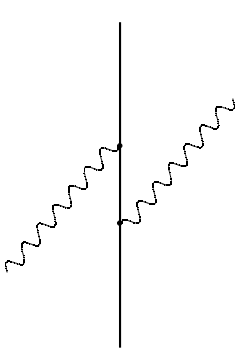

(b)

Figure 5. The two time-ordered diagrams required for light scattering. The incident light has wave-vector $\mathbf{k}$ and polarization $\lambda$ and is scattered (re-emitted) with wave-vector and polarization $\mathbf{k}^{\prime}$ and $\lambda^{\prime}$ respectively. The initial and final satate for the molecule is assumed to be the ground state 0 ; intermediate state carries the label $r$. 


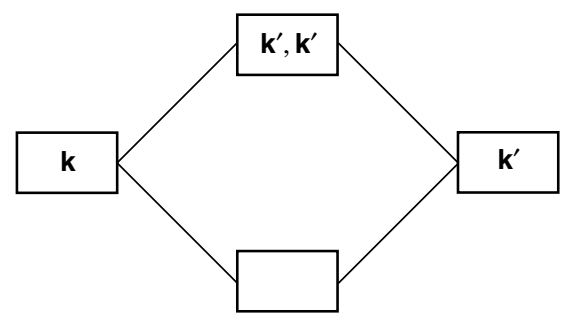

Figure 6. The state-sequence diagram for scattering.

example, from Fig. 5a, or the upper pathway in Fig. 6, we obtain the numerator $\left\langle f\left|\mu_{i}\right| r\right\rangle\left\langle r\left|\mu_{j}\right| i\right\rangle$. Calculation of each corresponding energy denominator using Eq. (74) requires us to identify the individual energy components. If we assume that the response is to be calculated for a molecule in its ground electronic state, then $E_{i_{\mathrm{mol}}}=E_{0}$ and the radiation field consists of $q$ photons of frequency $\omega$, $E_{i_{\text {rad }}}=q \hbar \omega$. The intermediate state energies, $\left(\tilde{E}_{r_{\text {mol }}}+E_{r_{\text {rad }}}\right)$, are again calculated with the aid of the diagrams. In the intermediate state of the coupled system in Fig. 5a, a photon has been absorbed by the molecule, which is thereby promoted to an intermediate electronic state-corresponding to the state box in the center of the lower pathway in Fig. 6. Therefore the total intermediate state energy is the sum of the intermediate molecular energy $\tilde{E}_{r_{\text {mol }}}$ and the modified radiation field $E_{r_{\text {rad }}}=(q-1) \hbar \omega$. Following Eq. (74), and considering only the first state sequence, we have as one contribution to the molecular polarizability:

$$
\alpha_{i j}^{(2 ; 1 a)}(-\omega ; \omega)=\sum_{r} \frac{\left\langle 0\left|\mu_{i}\right| r\right\rangle\left\langle r\left|\mu_{j}\right| 0\right\rangle}{\left(\tilde{E}_{r}-E_{0}+(q-1) \hbar \omega-q \hbar \omega\right)}=\sum_{r} \frac{\left\langle 0\left|\mu_{i}\right| r\right\rangle\left\langle r\left|\mu_{j}\right| 0\right\rangle}{\left(\tilde{E}_{r}-E_{0}-\hbar \omega\right)}
$$

Proceeding to evaluate in a similar manner the contribution associated with the alternative time ordering (Fig. 5b, and the upper pathway in Fig. 6), and then adding the result to (75), we arrive at the following final expression

$$
\alpha_{i j}^{(2)}(-\omega ; \omega)=\sum_{r}\left\{\frac{\left\langle 0\left|\mu_{i}\right| r\right\rangle\left\langle r\left|\mu_{j}\right| 0\right\rangle}{\left(\tilde{E}_{r 0}-\hbar \omega\right)}+\frac{\left\langle 0\left|\mu_{j}\right| r\right\rangle\left\langle r\left|\mu_{i}\right| 0\right\rangle}{\left(\tilde{E}_{r 0}+\hbar \omega\right)}\right\}
$$

using the standard energy difference notation $\tilde{E}_{r}-E_{0}=\tilde{E}_{r 0}$.

As an aside, it is useful to obtain from the Eq. (76) a result for the mean polarizability, whose value is required by Eq. (23). If the transition molecular dipoles are real (as is the case for nondegenerate transitions, or as may be enforced by a suitable choice of degenerate basis set), and the molecular 
environment is randomly oriented, an isotropic average can be employed, and the mean polarizability is then expressed as

$$
\begin{aligned}
\bar{\alpha}(-\omega ; \omega) & =\frac{1}{3} \sum_{r}\left\{\frac{|\langle r|\boldsymbol{\mu}| 0\rangle|^{2}}{\left(\tilde{E}_{r 0}-\hbar \omega\right)}+\frac{|\langle r|\boldsymbol{\mu}| 0\rangle|^{2}}{\left(\tilde{E}_{r 0}+\hbar \omega\right)}\right\} \\
& =\frac{2}{3} \sum_{r}|\langle r|\boldsymbol{\mu}| 0\rangle|^{2}\left\{\frac{\tilde{E}_{r 0}}{\tilde{E}_{r 0}^{2}-\hbar^{2} \omega^{2}}\right\}
\end{aligned}
$$

By explicitly including the wavevector and branch index dependence of the radiation frequency, and unfolding the detail of the molecular state damping, we conclude that the mean polarizability as used in Eq. (23) is

$$
\bar{\alpha}\left(\omega_{k}^{(m)}\right)=\frac{2}{3 \hbar} \sum_{r}\left\{\frac{|\langle r|\boldsymbol{\mu}| 0\rangle|^{2}\left(\Omega_{r 0}-\frac{i}{2} \gamma_{r}\right)}{\left(\Omega_{r 0}-\frac{i}{2} \gamma_{r}\right)^{2}-\omega_{k}^{(m)^{2}}}\right\}
$$

where by factorizing $\hbar$ from the expression, $\Omega_{r 0}$ represents each molecular frequency defined as $\Omega_{r 0}=\Omega_{r}-\Omega_{0}$. The imaginary elements in Eq. (78) arise from the substitution $\tilde{E}_{r}=E_{r}-\frac{1}{2} i \hbar \gamma_{r}$, to properly accommodate finite excitedstate lifetimes as discussed in the next section. It is represented explicitly here to illustrate that through Eqs. (78) and (23), the refractive index has both real and imaginary parts. The complex nature of (23), on passing through a particular molecular frequency, is illustrated in Fig. 7.

A second example, with $m=3$, illustrates the nonlinear molecular polarizability responsible for second-harmonic generation. Here each tensor numerator contains a product of three transition dipole moments. Reading off from the appropriate diagram, for example, using Fig. 2a, we obtain the numerator $\mu_{i}^{0 s} \mu_{j}^{s r} \mu_{k}^{r 0}$. Here we again assume that the molecule starts and finishes in its ground electronic state, and we have introduced the shorthand notation $\mu_{i}^{0 s} \mu_{j}^{s r} \mu_{k}^{r 0} \equiv\left\langle 0\left|\mu_{i}\right| s\right\rangle\left\langle s\left|\mu_{j}\right| r\right\rangle\left\langle r\left|\mu_{k}\right| 0\right\rangle$. Each denominator is a product of factors, one for each intermediate state, in each of which again the energy of the initial state is subtracted from the (complex) intermediate state energy. In the case of Fig. 2a, we find that for the intermediate state $|s\rangle$, the difference in molecular energies is $\tilde{E}_{s 0}\left(=\tilde{E}_{s}-\tilde{E}_{0}\right)$, and the difference in photon energies $-2 \hbar \omega$, thus giving a factor of $\left(\tilde{E}_{s 0}-2 \hbar \omega\right)$. For the intermediate state $|r\rangle$, the difference in molecular energies is $\tilde{E}_{r 0}$ and the difference in photon energies $-\hbar \omega$, giving a factor of $\left(\tilde{E}_{r 0}-\hbar \omega\right)$. Proceeding in a similar way from Figs. $2 \mathrm{~b}$ and $2 \mathrm{c}$ and summing, we thus obtain the following complete expression for the frequency- 


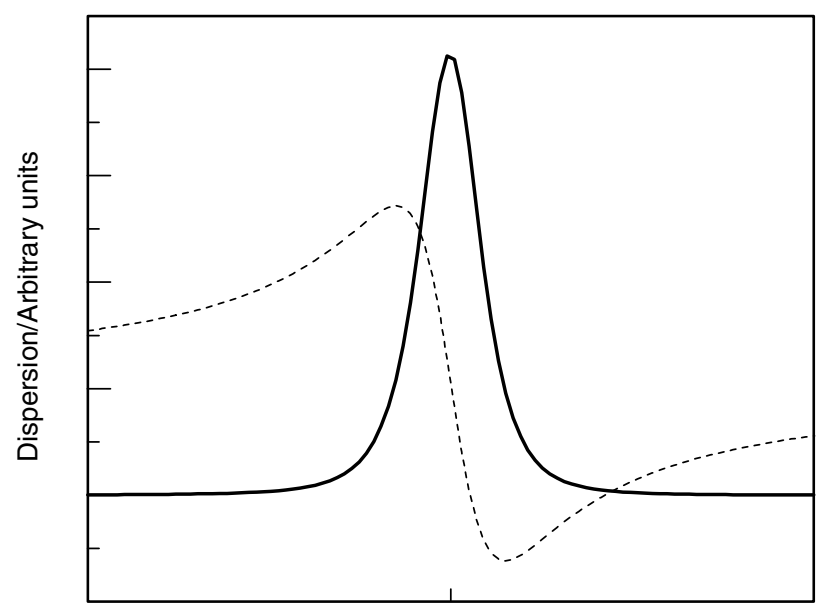

Frequency/Arbitrary units

Figure 7. The dotted line (the real part of Eq. (23) utilizing Eq. (78) as the mean polarizability) illustrates the dispresion of the refractive index across an arbitrary molecular resonance. The solid line represents the imaginary part of the refractive index and only contributes close to the resonant frequency.

doubling molecular polarizability tensor (the molecular hyperpolarizability)

$$
\begin{aligned}
\beta_{i j k}(-2 \omega ; \omega, \omega)= & \sum_{s} \sum_{r}\left[\frac{\mu_{i}^{0 s} \mu_{j}^{s r} \mu_{k}^{r 0}}{\left(\tilde{E}_{s 0}-2 \hbar \omega\right)\left(\tilde{E}_{r 0}-\hbar \omega\right)}+\frac{\mu_{j}^{0 s} \mu_{i}^{s r} \mu_{k}^{r 0}}{\left(\tilde{E}_{s 0}+\hbar \omega\right)\left(\tilde{E}_{r 0}-\hbar \omega\right)}\right. \\
& \left.+\frac{\mu_{j}^{0 s} \mu_{k}^{s r} \mu_{i}^{r 0}}{\left(\tilde{E}_{s 0}+\hbar \omega\right)\left(\tilde{E}_{r 0}+2 \hbar \omega\right)}\right]
\end{aligned}
$$

where we have used the common nomenclature to represent the leading order of nonlinear molecular response, specifically, $\boldsymbol{\beta}(-2 \omega ; \omega, \omega) \equiv \boldsymbol{\alpha}^{(3)}(-2 \omega ; \omega, \omega)$.

A few further general remarks are in order at this stage. One is to note the fact that the sum over intermediate molecular states, as in Eqs. (76) and (79), in principle applies not only to electronic but also to vibrational levels. Although this issue initially received most attention in connection with molecular hyperpolarizabilities [44], it applies equally to other optical response tensors. The vibrational contributions, which were previously largely overlooked, have now been extensively studied and shown to be important in many applications $[45,46]$. Second, the polarizabilities associated with nonlinear parametric processes may in most circumstances be regarded as properties of the groundstate molecule, since it is the molecular ground state that usually constitutes the 
initial and final molecular levels. Certainly it can be anticipated that under normal conditions the majority of conversion events will be mediated by ground (usually $S_{0}$ ) electronic state molecules, simply because of the overwhelming population of such molecules compared to those in excited states. However, other states may play the role of the initial/final state, and their corresponding polarizabilities can be evaluated in the same way. In fact, it transpires that the polarizabilities associated with electronic excited states can exceed those associated with the ground state by orders of magnitude, as has been shown both in theory and experiment [47-49]. Thus, if the appropriate excited state is optically pumped so as to provide a significant population of molecules, the observed polarizability characteristics of the medium can be significantly enhanced, or diminished. This is an important fact that we shall return to when discussing optically induced harmonic generation in more detail.

\section{DAMPING}

The issue of correctly signing the damping of energy denominators in optical response tensors has been the subject of much recent debate [see, e.g., Refs. 5052]. This stems partly from a common confusion in the literature between two entirely different forms of damping; it also reflects attempts to impose conflicting conditions on the molecular response. The former obscurity is very easily dealt with, though; as will be shown below, it forms the ground in which seeds of the latter conflict have been sown.

First, the emergence of photon (more accurately, bath quantum, polariton) energies in the denominators of expressions such as Eqs. (76) and (79) originated in the development of the signal amplitude from Eq. (36). In the evaluation of the underlying contour integrals invoked at that stage, imaginary infinitesimals are commonly added to the photon energies to displace the poles from the real axis. Each photon energy thereby acquires an infinitesimal addendum, $\hbar \omega \rightarrow \hbar \omega+i s$, with $s \rightarrow+0$. In the polarizability equation (76), for example, this modification introduces addenda of $-i s$ and $+i s$, respectively, to the two energy denominators-a prescription that also allows the tensor to retain the property of hermiticity. It has to be emphasized, nonetheless, that the result has no meaning other than in the specific case of the limit $s \rightarrow+0$.

The second type of resonance modification to energy denominators, alluded to earlier, is designed to reflect the finite lifetime of each molecular energy level, phenomenologically implemented by a modification of the corresponding energy

$$
\tilde{E}_{r}=E_{r} \pm \frac{1}{2} i \hbar \gamma_{r}
$$

where $\gamma_{r}$ may be considered a sum of the inverse lifetimes associated with each line-broadening mechanism, and representing the FWHM (full width at 
half-maximum) linewidth of the nonlinear response near resonance. Only the ground state is undamped, since its lifetime is taken as infinite. Using the positive sign in equation (80) results in the time dependence of each molecular state $|r\rangle$ acquiring within its phase factor $e^{-i \tilde{E}_{r} t / \hbar}$ an exponential decay component. More significantly, the choice of the positive sign for the damping proves to be uniformly consistent with time-reversal symmetry, as will be discussed in more detail below. The result of implementing this correction in the polarizability equation (76), for example, is the addition of $+\frac{1}{2} i \hbar \gamma_{r}$ to each energy denominator, the sign the same in each term. In nonresonant processes, $\hbar \gamma_{r}$ is typically several orders of magnitude smaller than $E_{r}$, and its precise value is determined by the nature of the molecule and the local structure of the bulk phase. Near to resonance, the damping serves to give a realistic lineshape to the optical response. For example, in light scattering close to an optical absorption band where $\hbar \omega \approx E_{r 0}$, the first term of Eq. (76) dominates and the corresponding rate acquires a Lorentzian lineshape. Resonance features play a particularly prominent role in the case of many large organic structures, whose ultrafast excited-state decay mechanisms produce damping factors on the terahertz scale. Detailed consideration of lineshape is also necessary in order to properly accommodate the dispersion behavior featured in the realization of wavevector matching for parametric processes.

Historically two conventions have been used extensively in the literature for setting the signs of the phenomenological damping factors. Although mutually incompatible, justifications for each convention have been made by appeal to causality - a different line generally being taken in the semiclassical and fully quantum-mechanical approaches to the interacting system of molecules and radiation. In earlier work [1] this issue was unresolved and the prevailing convention (variable signing, discussed in the following paragraphs) was adopted. Now it is clear that constant signing is correct; an example is instructive. The two conventions with respect to second-harmonic generation are as follows; in the semiclassical or variable-sign convention (vsc) the signs are chosen oppositely for interactions preceding and following in time the emission of the harmonic photon, as follows [53-55]:

$$
\begin{aligned}
\beta_{i j k}^{v s c}(-2 \omega ; \omega, \omega)= & \sum_{s} \sum_{r}\left[\frac{\mu_{i}^{0 s} \mu_{j}^{s r} \mu_{k}^{r 0}}{\left(E_{s 0}-2 \hbar \omega-\frac{1}{2} i \hbar \gamma_{s}\right)\left(E_{r 0}-\hbar \omega-\frac{1}{2} i \hbar \gamma_{r}\right)}\right. \\
& +\frac{\mu_{j}^{0 s} \mu_{i}^{s r} \mu_{k}^{r 0}}{\left(E_{s 0}+\hbar \omega+\frac{1}{2} i \hbar \gamma_{s}\right)\left(E_{r 0}-\hbar \omega-\frac{1}{2} i \hbar \gamma_{r}\right)} \\
& \left.+\frac{\mu_{j}^{0 s} \mu_{k}^{s r} \mu_{i}^{r 0}}{\left(E_{s 0}+\hbar \omega+\frac{1}{2} i \hbar \gamma_{s}\right)\left(E_{r 0}+2 \hbar \omega+\frac{1}{2} i \hbar \gamma_{r}\right)}\right]
\end{aligned}
$$


In the fully quantum-mechanical development [constant-sign convention, (csc)], as adopted in most of the literature on Raman scattering, all the signs of the damping are identical:

$$
\begin{aligned}
\beta_{i j k}^{\mathrm{csc}}(-2 \omega ; \omega, \omega)= & \sum_{s} \sum_{r}\left[\frac{\mu_{i}^{0 s} \mu_{j}^{s r} \mu_{k}^{r 0}}{\left(E_{s 0}-2 \hbar \omega-\frac{1}{2} i \hbar \gamma_{s}\right)\left(E_{r 0}-\hbar \omega-\frac{1}{2} i \hbar \gamma_{r}\right)}\right. \\
& +\frac{\mu_{j}^{0 s} \mu_{i}^{s r} \mu_{k}^{r 0}}{\left(E_{s 0}+\hbar \omega-\frac{1}{2} i \hbar \gamma_{s}\right)\left(E_{r 0}-\hbar \omega-\frac{1}{2} i \hbar \gamma_{r}\right)} \\
& \left.+\frac{\mu_{j}^{0 s} \mu_{k}^{s r} \mu_{i}^{r 0}}{\left(E_{s 0}+\hbar \omega-\frac{1}{2} i \hbar \gamma_{s}\right)\left(E_{r 0}+2 \hbar \omega-\frac{1}{2} i \hbar \gamma_{r}\right)}\right]
\end{aligned}
$$

The latter result (82) yields a quantum probability amplitude that, under Hermitian conjugation and time reversal, correctly equates to the corresponding amplitude for the time-inverse process of degenerate downconversion. To see this, we note that the matrix element for SHG invokes the tensor product $\beta_{i j k}(-2 \omega ; \omega, \omega) \rho_{i(j k)}$, where the brackets embracing two of the subscripts $(j k)$ in the radiation tensor denote index symmetry, reflecting the equivalence of the two input photons. As shown previously [1], this allows the tensor product to be written without loss of generality as $\beta_{i(j k)}(-2 \omega ; \omega, \omega) \rho_{i(j k)}$, entailing an indexsymmetrized form of the molecular response tensor,

$$
\beta_{i(j k)}(-2 \omega ; \omega, \omega)=\frac{1}{2}\left\{\beta_{i j k}(-2 \omega ; \omega, \omega)+\beta_{i k j}(-2 \omega ; \omega, \omega)\right\}
$$

Each of the six terms of the hyperpolarizability tensor so formed transforms into one of the six counterpart terms in $\beta_{i(j k)}(2 \omega,-\omega,-\omega)$, the tensor for degenerate downconversion, on performing the combined operations of Hermitian conjugation and time reversal (the radiation tensor for downconversion is also obtained by performing the same procedure on $\left.\rho_{i(j k)}\right)$. For example, the last term of $\beta_{i(j k)}(-2 \omega ; \omega, \omega)$, in the order that logically follows from Eqs. (82) and (83), behaves as follows:

$$
\begin{array}{r}
\frac{\mu_{k}^{0 s} \mu_{j}^{s r} \mu_{i}^{r 0}}{\left(E_{s 0}+\hbar \omega-\frac{1}{2} i \hbar \gamma_{s}\right)\left(E_{r 0}+2 \hbar \omega-\frac{1}{2} i \hbar \gamma_{r}\right)} \\
\stackrel{H T}{\longrightarrow} \frac{\mu_{i}^{0 r} \mu_{j}^{r s} \mu_{k}^{s 0}}{\left(E_{s 0}+\hbar \omega-\frac{1}{2} i \hbar \gamma_{s}\right)\left(E_{r 0}+2 \hbar \omega-\frac{1}{2} i \hbar \gamma_{r}\right)}
\end{array}
$$

and on interchanging the dummy state sum indices $r$ and $s$, the result is exactly the first term of $\beta_{i j k}(2 \omega ;-\omega,-\omega)$ as follows from the form given by (82). The 


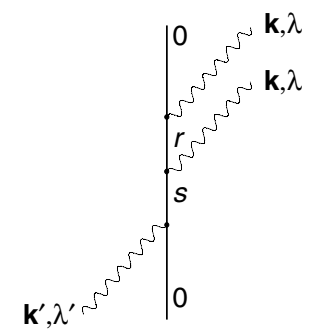

(a)

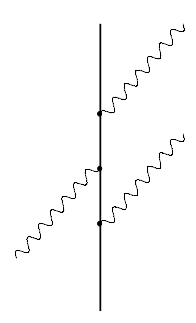

(b)

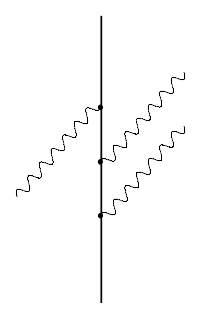

(c)

Figure 8. The three time-ordered diagrams for degenerate down-conversion, representing exact time reversal of the SHG orderings illustrated in Fig. 2. Consequently the emitted photons now carry the wave vector and polarization labels, $\mathbf{k}$ and $\lambda$, and the annihilated photon is characterized by $\mathbf{k}^{\prime}, \lambda^{\prime}$.

time reversal represented above is depicted in the time-ordered diagrams of Fig. 8. The two-step operation of $H T$ is given above to clarify the action of the two operators, although it introduces assumptions that the indices relate to contractions with real polarization vectors and that the molecular states are all nondegenerate and time-even, consistent with spin-paired molecular orbitals. However, the combined operation is not subject to these conditions, so that the end result holds in general $[11,24,50]$.

The variable-sign result Eq. (81) produces results that fail to satisfy such time-reversal symmetry, as shown by Andrews et al. [50]. The requirement for temporal symmetry remains unequivocal, despite the violation of time-reversal invariance by the system itself (its engagement of molecular interaction with the bath leading to state decay), specifically because of the inclusion of damping. The two conventions agree in ostensibly the most crucial signing, that which relates to potentially resonant denominator terms; they differ in "antiresonant" terms. Nonetheless, in certain processes they can lead to results with experimentally very significant differences.

The origin of confusion surrounding the correct form of damping is readily identified on comparison of Eqs. (81) and (82). In the latter, consistent signing is associated with the consistently positive signs of the energies $E_{s 0}$ and $E_{r 0}$; in the former, the signing appears consistent with the variable signs of the photon energies $\hbar \omega, 2 \hbar \omega$. Thus, if the imaginary infinitesimals discussed earlier are directly substituted by physically meaningful and finite damping constants, spurious results conforming to the variable-sign rule emerge. Those results satisfy the Hermitian property of reciprocity, but that is not a principle of universal application [56]. Any prescription with variable assignment of signs can also introduce significant ambiguities in connection with processes entailing two or more outgoing waves, as, for example, in four-wave mixing. 
The variable-sign convention would also logically lead to a secondary rule, that where static fields appear in electro- or magneto-optical processes, the interactions with which they are associated should carry no damping. However, from a quantum field theoretic viewpoint, static perturbations must induce damping [52]. All electromagnetic interactions are fundamentally mediated through the exchange of virtual photons (the gauge bosons). A static field involved in an electro-optical process in any given molecule is mediated in the same way. It owes its origin to the coupling between the charges within that molecule and those constituting the source of the static field. This coupling is expressed through the accommodation of interactions with virtual photons from modes of an infinite range, as with any electrodynamic interaction, and summation over the virtual photon wavevectors and polarizations thereby ensures a result that properly reflects the conservation of energy. Consequently, the case of a static field is no different in type from a time-varying field-except that, while causality is, of course, satisfied, explicit retardation features disappear. Hence the damping associated with any molecular excited state must be subject to damping, irrespective of the frequency of the electric field responsible for the perturbation. Damping factors are not frequency-dependent; each excited state has a damping of a characteristic magnitude, irrespective of the frequency of the perturbation with which it is associated. In this connection it has been shown that the correct constant-signing rule is necessary to uphold the principle that linear electro-optical response cannot occur in an isotropic liquid, whether chiral or not [57].

In general, detailed consideration of damping is especially important when operating near to resonance, which in general occurs when there exists a molecular state differing in energy from the initial state by an amount approaching the energy of one or more of the photons involved. For coherent parametric processes the need to operate in regions of dispersion, in order to satisfy wavevector matching conditions, is a well-known experimental technique. Operating in such frequency regions necessitates adoption of the polariton (rather than vacuum photon) formulation, as described in Section III. Inspection of the dispersion curves in Fig. 1, considering for simplicity case (a) with just one molecular frequency, clearly illustrates three areas of interest: (1) the diagonal curve segments represent photon-like radiation propagating through the media at transparent frequencies, (2) the horizontal regions exhibit excitonlike molecular resonances (photons impinging on the medium at such frequencies are readily absorbed into the molecular bulk), and (3) level-crossing areas signify a dispersive mixing of the molecular and radiation states. It is these latter regions in which it is commonly necessary to operate. Thus, depending on whether the radiation frequency is sufficiently above or below a particular molecular resonance will determine the branch index that is appropriate. 


\section{INDEX SYMMETRY AND MOLECULAR STRUCTURE}

A number of symmetry factors of quite distinct origins play a significant role in determining the nullity or nonnullity of the various components of any molecular optical response tensor ( $3^{n}$ components in the case of dipole coupling). Equally, symmetry considerations determine the number of linearly independent components. In second-harmonic generation, for example, the symmetrized tensor components must satisfy identities such as $\beta_{z(x y)}=\beta_{z(y x)}$, regardless of whether the molecule possesses the three- or higher-fold axis of symmetry necessary to confer degeneracy on the $x$ and $y$ axes. Consequently, of the $3^{3}=27$ tensor components, only 18 are independent. In general, for $n$-harmonic processes, the rigorously index-symmetric polarizability tensor has only $3(n+1)(n+2) / 2$ independent components. The inherent structural symmetry of most molecules generally reduces this number still further.

It is most important to note that in many cases of harmonic emission, a more completely index-symmetric form of the polarizability tensor is implicated. Consider once again the prototypical example of optical nonlinearity afforded by harmonic generation. When any harmonic is generated from a planepolarized beam, in an isotropic medium, it produces photons with the same polarization vector as the incident light. In such a case the radiation tensor $\rho_{i j k}$ becomes fully index-symmetric, and arguments similar to those given above show that only the fully index-symmetric part of the hyperpolarizability tensor, $\beta_{i j k}(-2 \omega ; \omega, \omega)$, can be involved. This does not mean that the tensor itself is inherently fully index-symmetric, but it does mean that experiments of the kind described cannot determine the extent of any index antisymmetry.

This leads us to the issue of approximate index symmetry. Any nonlinear optical process involving only static (zero-frequency) fields is correctly described in terms of a classical response tensor, which is always fully indexsymmetric. When optical frequencies are involved, the differences between the energy denominators of the various terms in the tensor remove this symmetry. The only exception, albeit an important one, is the linear polarizability. Since this entails the product of two transition moments connecting the same pair of states, permutational index symmetry is guaranteed. If, however, the photon frequencies all fall substantially below any electronic transition frequencies of the material, all energy denominators become approximately equal, $\left(\tilde{E}_{s 0} \approx \tilde{E}_{r 0}\right.$ in the case of frequency doubling), and the tensor becomes in effect fully indexsymmetric. Under such conditions the polarizability tensor for an $n$-harmonic process has only $(n+2)(n+3) / 2$ independent components, for example, 10 in the case of frequency doubling. Index symmetry based on the assumption that such an approximation is valid, normally referred to as Kleinman symmetry [58] is nonetheless unjustified in a great many applications $[43,59]$. 
We now establish on the basis of physical symmetry the conditions for the existence of the $m$-photon optical response tensor $\boldsymbol{\alpha}^{(m)}$. For any parametric process, the initial and final molecular states are identical and normally carry the full ground-state symmetry of the molecule (or, in the case of a crystalline solid, that of the unit cell). Thus, since each term in the explicit expression (74) contains a product of $m$ transition moments, the tensor can be nonvanishing only if the totally symmetric representation of the appropriate point group or space group is spanned by the product of $(n+1)$ translations (in the case of electric dipole, E1, coupling). The origin of this condition can be traced back to Eq. (41). Where the molecule or crystal possesses a center of symmetry, the symmetry condition can be met only in the generation of odd harmonics, where $m$ is even and the product of translations is thus of gerade (even) symmetry. For the same reason free atoms cannot support the production of even harmonics, except under special conditions that effectively disrupt atomic symmetry.

Much more detailed symmetry information follows by considering the explicit group-theoretic basis. In general, the independent components of the response tensor form a basis for a reducible representation of the appropriate molecular or crystallographic group; the reduction of this representation into irreducible parts leads to results such as those given in Table I, in which $D^{(j p)}$ stands for the irreducible representation of weight $j$ and parity $p$. In this table, based on the transformation properties associated the normal E1 coupling, the second-harmonic polarizability $\boldsymbol{\beta}(-2 \omega ; \omega, \omega)$ is represented as $\boldsymbol{\beta}^{\mathrm{SHG}}$ and the third-harmonic as $\gamma^{\mathrm{THG}} \equiv \gamma(-3 \omega ; \omega, \omega, \omega)$, although in each case it should be borne in mind that the same molecular tensors are involved in both coherent and incoherent harmonic processes. The first entry for each tensor gives the reduction based on consideration of only the index symmetry inherent in the interaction, as, for example, is denoted by the brackets around the subscripts in $\beta_{i(j k)}^{\mathrm{SHG}}$ : the second entries give the results that apply under Kleinman assumption of full index symmetry, such as $\beta_{(i j k)}^{\mathrm{SHG}}$. In each case the entry in the final column gives $r$, the number of independent tensor components. This may be regarded as

TABLE I

Group-Theoretic Representations and Number of Components $r$ of Second- and Third-Harmonic Polarizabilities

\begin{tabular}{lllc}
\hline Coupling & Tensor & \multicolumn{1}{c}{ Representation } & $r$ \\
\hline $\mathrm{E} 1\left(\mathrm{E} 1^{2}\right)$ & $\beta_{i(j k)}^{\mathrm{SHG}}$ & $2 D^{(1-)} \oplus D^{(2-)} \oplus D^{(3-)}$ & 18 \\
$\left(\mathrm{E} 1^{3}\right)$ & $\beta_{(i j k)}^{\mathrm{SHG}}$ & $D^{(1-)} \oplus D^{(3-)}$ & 10 \\
$\mathrm{E} 1\left(\mathrm{E} 1^{3}\right)$ & $\gamma_{i(j k l)}^{\mathrm{THG}}$ & $D^{(0+)} \oplus D^{(1+)} \oplus 2 D^{(2+)} \oplus D^{(3+)} \oplus D^{(4+)}$ & 30 \\
$\left(\mathrm{E} 1^{4}\right)$ & $\gamma_{(i j k l)}^{\mathrm{THG}}$ & $D^{(0+)} \oplus D^{(2+)} \oplus D^{(4+)}$ & 15 \\
\hline
\end{tabular}


a maximum, applicable to molecules totally lacking any intrinsic symmetry; any molecule with a twofold or higher axis of symmetry will inevitably manifest other relationships between its tensor components which will further reduce the number of independent parameters.

Any parametric nonlinear polarizability can be supported by a given molecule or crystal only as long as the totally symmetric representation of the corresponding point or space group is spanned by some components of the tensor. This remains true even for the parametric polarizabilities associated with molecular excited states; provided they are nondegenerate, the product of the initial and final state representations will generate the totally symmetric representation. In the very rare cases where the polarizability properties of molecules in degenerate excited states are required, the theory can be extended in an obvious way. Since components that transform under $D^{(0+)}$ invariably span the totally symmetric representation, it is immediately apparent from Table I that, on the basis of molecular symmetry, third-harmonic processes are universally allowed. Indeed, this is true for all odd harmonics; it is because the corresponding polarizabilities invariably carry components of $D^{(0+)}$ symmetry.

By contrast in the case of SHG, for example, only those species whose totally symmetric representation is spanned by $D^{(1-)}, D^{(2-)}$, or $D^{(3-)}$ components can support the hyperpolarizability tensor $\boldsymbol{\beta}^{S H G}$, and this excludes all centrosymmetric species. More interestingly, the condition is satisfied by all polar species, since these necessarily have $D^{(1-)}$ components transforming under the totally symmetric representation. In fact, among the common nonpolar molecular point groups, only the following permit a nonzero hyperpolarizability, by virtue of having $D^{(2-)}$ and/or $D^{(3-)}$ components transforming under their totally symmetric representation: $S_{4} ; C_{3 h} ; D_{2} ; D_{3} ; D_{4} ; D_{6} ; D_{3 h} ; D_{2 d} ; T ; T_{d}$ [60]. If Kleinman symmetry is assumed, the hyperpolarizability tensor carries only $D^{(1-)}$ and $D^{(3-)}$ components: in this case the SHG potential of species with $D_{4}$ or $D_{6}$ symmetry, which have only $D^{(2-)}$ components, is not apparent.

Although index symmetry places constraints on the maximum number of independent elements for any given nonlinear susceptibility, that number is generally reduced to a significant degree by virtue of molecule, crystal, or site symmetry, where present. Two features are responsible for this; one is the fact that symmetry will generally dictate that certain tensor components are necessarily zero. For example, in any species with a mirror plane perpendicular to the $z$ axis $\beta_{z(z z)}^{\text {SHG }}$, must be zero since it has to equal its own negative. Second, structural symmetry will usually forge relationships between different components. For example, in species with a threefold axis of proper rotational symmetry, $\beta_{x(x x)}^{\mathrm{SHG}}$ has to equal minus $\beta_{x(y y)}^{\mathrm{SHG}}$. The explanation is that the product $x\left(x^{2}+y^{2}\right)$ does not transform under the totally symmetric representation, and hence the linear combination $\left(\beta_{x(x x)}^{\mathrm{SHG}}+\beta_{x(y y)}^{\mathrm{SHG}}\right)$ must be zero. For such reasons the 
18 tensor components associated with SHG generally reduces to a far smaller number; in the extreme case of species with tetrahedral symmetry, there is in fact only one independent component $(x y z)$.

Where local symmetry permits harmonic generation based exclusively on electric dipole coupling, the inclusion of higher-order multipolar contributions in the exact coupling equation (13) produces additional terms that are normally negligible. If electric dipole harmonic generation is forbidden, which, as we have seen, is the case for even harmonics in a centrosymmetric species, these higher-order terms can nonetheless become significant and may operate to effect weak harmonic emission. The exception is the case of an isotropic fluid, where global symmetry precludes the involvement of any higher multipoles in secondharmonic emission within the bulk [61-63]. Work by Cao and Zhu [64] has shown how the multipolar generation of a second harmonic signal in such systems necessitates the presence of a surface, from which the harmonic can emerge as a reflected beam. In other systems lacking full rotational symmetry, the higher multipoles can also be important. For example, if any one of the three ungerade electric dipole (E1) interactions involved in second-harmonic generation is replaced by a gerade electric quadrupole (E2) interaction, the operator product generates a $D^{(0+)}$ contribution as shown in Table II. Since this invariably spans the totally symmetric representation, the corresponding response tensor is nonzero even in centrosymmetric materials. In the first row of Table II the coupling E1(E1E2) refers to the polarizability associated with one electric dipole and one electric quadrupole annihilation of a pump photon, with electric dipole emission of the harmonic. The labeling $\mathrm{E} 2\left(\mathrm{E} 1^{2}\right)$ in the second row relates to electric dipole annihilation of both pump photons and electric quadrupolar harmonic emission. In the third row the coupling $\left(\mathrm{E} 1^{2} \mathrm{E} 2\right)$ can refer to either case, but the corresponding representation applies only under

TABLE II

Representations and Number of Components $r$ of Leading Higher-Order Multipole SecondHarmonic Polarizabilities

\begin{tabular}{llll}
\hline Coupling & Tensor & \multicolumn{1}{c}{ Representation } & $r$ \\
\hline E1(E1E2) & $\tilde{\beta}_{i(j k) l}^{\text {SHG }}$ & $D^{(0+)} \oplus 2 D^{(1+)} \oplus 3 D^{(2+)} \oplus 2 D^{(3+)} \oplus D^{(4+)}$ & 45 \\
E2(E1 $\left.{ }^{2}\right)$ & $\tilde{\beta}_{(i j)(l k)}^{\text {SHG }}$ & $D^{(0+)} \oplus D^{(1+)} \oplus 2 D^{(2+)} \oplus D^{(3+)} \oplus D^{(4+)}$ & 30 \\
$\left(\mathrm{E} 1^{2}\right.$ E2) & $\tilde{\beta}_{(i j k l)}^{\text {SHG }}$ & $D^{(0+)} \oplus D^{(2+)} \oplus D^{(4+)}$ & 15 \\
E1(E1M1) & $\beta_{i j k}^{\text {SHG }}$ & $D^{(0+)} \oplus 3 D^{(1+)} \oplus 2 D^{(2+)} \oplus D^{(3+)}$ & 27 \\
M1(E1 $\left.{ }^{2}\right)$ & $\beta_{i(j k)}^{\text {SHG }}$ & $2 D^{(1+)} \oplus D^{(2+)} \oplus D^{(3+)}$ & 18 \\
$\left(\right.$ E1 ${ }^{2}$ M1 $)$ & $\beta_{(i j k)}^{\text {SHG }}$ & $D^{(1+)} \oplus D^{(3+)}$ & 10 \\
\hline
\end{tabular}


the assumption of Kleinman index symmetry. Similar remarks apply if one of the electric dipole couplings is alternatively replaced by a gerade magnetic dipole (M1) interaction, though in this case the $D^{(0+)}$ representation arises only where the magnetic interaction is involved in the annihilation of a pump photon. Moreover, the $D^{(0+)}$ feature is not apparent if Kleinman symmetry is assumed.

\section{TWO-LEVEL SYSTEMS}

From the results in the last section it is clear that for particular applied radiative frequencies or frequency multiples, close to resonance with particular molecular states, each molecular tensor will be dominated by certain terms in the summation of states as a result of their diminished denominators-a principle that also applies to all other multiphoton interactions. This invites the possibility of excluding, in the sum over molecular states, certain states that much less significantly contribute. Then it is expedient to replace the infinite sum over all molecular states by a sum over a finite set-this is the technique employed by computational molecular modelers, their results often producing excellent theoretical data. In the pursuit of analytical results for near-resonance behavior, it is often defensible to further limit the sum over states and consider just the ground and one electronically excited state. Indeed, the literature is replete with calculations based on two-level approximations to simplify the optical properties of complex molecular systems. On the other hand, the coherence features that arise through adoption of the celebrated Bloch equations are limited to exact two-level systems and are rarely applicable to the optical response of complex molecular media.

In the case of a single resonance, optical harmonic conversion is driven largely by transitions involving just the ground and resonant levels, so that the kinetics of the process approximates that of a two-level system. Indeed, in the realm of resonant multiphoton phenomena the two-level approximation is peculiarly appropriate for harmonic emission, whereas most nonparametric processes such as multiphoton absorption require three or more levels for their adequate representation. Consider once again, for example, the case of frequency doubling, where resonance amplification can occur at either the pump or the harmonic frequency. To begin, it is useful to separate the molecular tensor into a sum of two parts, in the first of which both the summations over intermediate states $|r\rangle$ and $|s\rangle$ are restricted to the ground level $|0\rangle$ and a resonant level $|u\rangle$; in the second, all other possibilities are accounted for. Hence we can write

$$
\beta_{i j k}(-2 \omega ; \omega, \omega)=\beta_{i j k}^{\mathrm{TLA}}(-2 \omega ; \omega, \omega)+\beta_{i j k}^{\text {others }}(-2 \omega ; \omega, \omega)
$$


where the superscript "TLA" denotes the two-level approximation. It is to be noted at the outset that the $\boldsymbol{\beta}^{\text {others }}$ term does not completely exclude the states $|0\rangle$ and $|u\rangle$ from every intermediate state summation; for example, it accommodates contributions associated with $|r\rangle=|u\rangle,|s\rangle \neq\{|0\rangle,|u\rangle\}$.

Focusing first on the dominant two-level term [65], careful analysis of the tensor structure, with respect to the proper signs for the damping corrections and utilizing the freedom to add a $j, k$ index-antisymmetric term (see later), yields the following result $[59,66]$ :

$$
\begin{aligned}
\beta_{i j k}^{\mathrm{TLA}}(-2 \omega ; \omega, \omega)= & {\left[\frac{\mu_{i}^{0 u} \mu_{j}^{0 u} \breve{d}_{k}}{\left(E_{u 0}-2 \hbar \omega-i \Gamma_{u}\right)\left(E_{u 0}-\hbar \omega-i \Gamma_{u}\right)}\right.} \\
& +\frac{\mu_{j}^{0 u} \breve{d}_{j} \mu_{k}^{0 u}}{\left(E_{u 0}+\hbar \omega-i \Gamma_{u}\right)\left(E_{u 0}-\hbar \omega-i \Gamma_{u}\right)} \\
& \left.+\frac{\breve{d}_{j} \mu_{k}^{0 u} \mu_{i}^{0 u}}{\left(E_{u 0}+\hbar \omega-i \Gamma_{u}\right)\left(E_{u 0}+2 \hbar \omega-i \Gamma_{u}\right)}\right]
\end{aligned}
$$

assuming only that the electric dipole transition moments are real, and for conciseness introducing the shorthand notation $\Gamma_{u}=\frac{1}{2} \hbar \gamma_{u}$. Where only diagonal components arise, as, for example, may apply for harmonic generation within a regular solid, the tensor product $\boldsymbol{\beta}^{T L A} \cdot \boldsymbol{\sigma}$ featured in the rate equation (51) reduces to a simpler structure first identified by Oudar and Chemla [67]. Both in its simpler form, and in the general expression Eq. (85), the most significant feature is the appearance in each term of the vector parameter $\breve{\mathbf{d}}$, defined by

$$
\breve{\mathbf{d}}=\boldsymbol{\mu}^{u u}-\boldsymbol{\mu}^{00}
$$

specifically, the difference between the static electric dipole moments of the resonant and ground states. Hence the two-level hyperpolarizability displays a linear dependence on the magnitude of $\breve{\mathbf{d}}$, which, for example, in extensively conjugated molecules can reasonably be assumed proportional to the length of the conjugation chain [67].

It is important to have included the ground state of the molecule in the sums over intermediate states for the dependence on $\breve{\mathbf{d}}$ to be recovered, and this proves significant for two reasons: (1) the result Eq. (85) shows that the twolevel hyperpolarizability can be supported only by molecules with permanent ground- or excited-state dipoles, which means polar molecules; and (2) it is clear that there is considerable scope for the two-level response to be enhanced in polar molecules having a resonant excited state whose equilibrium geometry is appreciably different from that of the ground state, or in species exhibiting the characteristically strong absorption associated with a charge-transfer transition. 
Similar features arise in the theory of multiphoton absorption [68-70] and also single-photon cooperative absorption [71,72]. In connection with secondharmonic generation, the result has added significance since most species that can support $\boldsymbol{\beta}(-2 \omega ; \omega, \omega)$, and thereby have the potential for frequency doubling, are, indeed, polar (see previous section).

Let us now consider more specifically the case of a medium possessing an excited state $|u\rangle$ close in energy to that of the emitted harmonic, $2 \hbar \omega$. For practical application, this condition is generally more useful than resonance at the fundamental frequency, since the latter condition is likely to result in a substantial loss of pump power through conventional single-photon absorption. In view of its denominator structure, it is clearly the first term in Eq. (85) that will provide the major contribution to the nonlinear response tensor

$$
\beta_{i j k}^{\mathrm{TLA}} \approx \frac{\mu_{i}^{0 u} \mu_{j}^{0 u} \breve{d}_{k}}{\left(\hbar \Delta \omega-i \Gamma_{u}\right)\left(\hbar \omega+\hbar \Delta \omega-i \Gamma_{u}\right)}
$$

where $\Delta \omega$ represents the detuning from resonance: both the first and second terms of Eq. (85) dominate in the case of resonance at the fundamental frequency. If the molecule has no dipole and possesses a center of symmetry, it is well known that the all hyperpolarizability tensor components are null and no second-harmonic generation is possible. However, if only $\mathbf{d}$ vanishes, as in the case of a tetrahedrally symmetric molecule, then only the two-level contribution to the tensor, $\boldsymbol{\beta}^{\mathrm{TLA}}(-2 \omega ; \omega, \omega)$, disappears. The remaining contribution $\boldsymbol{\beta}^{\text {other }}(-2 \omega ; \omega, \omega)$, as defined by Eq. (84), persists and is itself dominated by a term with essentially the same denominator structure as Eq. (87); specifically, the product of a near-resonant and an off-resonant term. For the general structure that then emerges, the reader is referred to Andrews [1].

It has been established in a series of works that a transformation of the electric dipole interaction is valid for deriving the optical characteristics of molecular systems with a response dominated by two electronic states [68-70; 73-77]. This procedure relates to the employment of a fluctuation dipole operator $[78,79]$ as given by

$$
H_{\text {int }}^{\prime}=-\varepsilon_{0}^{-1}\left[\boldsymbol{\mu}-\boldsymbol{\mu}^{00}\right] \cdot \mathbf{d}^{\perp}
$$

in which the subtracted moment is the permanent dipole of the initial molecular state-usually the ground state. It has been proved how utilizing Eq. (88) for the form of the interaction operator leads to a new and expedient algorithm for the calculation of the requisite nonlinear optical polarizabilities [80], based on a novel interpretation of the appropriate time-ordered diagrams. In establishing the form for probability amplitudes of systems driven primarily by interactions 
between the ground state $|0\rangle$, and one other higher electronic state $|u\rangle$, it proves legitimate and expedient simply to recast the permanent moments, where they arise as follows

$$
\boldsymbol{\mu}^{u u} \rightarrow \boldsymbol{\mu}^{u u}-\boldsymbol{\mu}^{00}=\breve{\mathbf{d}} ; \quad \boldsymbol{\mu}^{00} \rightarrow 0
$$

while leaving any transition dipoles $\left(\boldsymbol{\mu}^{u 0}, \boldsymbol{\mu}^{0 u}\right)$ unchanged. When the various time orderings for any optical processes of interest are drawn up, application of this rule enables expressions involving any connected route that entails the groundstate dipole $\boldsymbol{\mu}^{00}$ to be discarded, so long as those entailing the excited state dipole $\boldsymbol{\mu}^{u u}$ are re-interpreted to invoke $\breve{\mathbf{d}}$. This is the algorithm whose illustrative applications are described below. The method has been explicitly validated for all parametric and nonparametric processes, both degenerate and fully nondegenerate [80] and can be shown to correspond to a canonical transformation on the interaction Hamiltonian [14a,b; 81]. In every case its implementation leads in a matter of lines to results identical to those previously established by substantially more laborious means $[66,69,70]$. It may also be noted that the fluctuation dipole results are exact, when the correct constant-sign convention is used for damping; when variable signing is employed, the result is approximate only [66].

As an example, we again derive the two-level second harmonic tensor, this time using the algorithm outlined by Eq. (89). The three time-ordered diagrams as illustrated in Fig. 2a-c are once more employed. The route of molecular states between the initial (ground) state and the final (also ground) state runs through two virtual states, $|r\rangle$ and $|s\rangle$, and in the two-level approximation each of these is summed to represent one of two possibilities, either the ground state $|0\rangle$ or the excited state $|u\rangle$. The $(|0\rangle \leftarrow|s\rangle \leftarrow|r\rangle \leftarrow|0\rangle)$ sequences that arise are thus concisely expressible as $0000,00 u 0,0 u 00,0 u u 0$, corresponding to the dipole products $\boldsymbol{\mu}^{00} \boldsymbol{\mu}^{00} \boldsymbol{\mu}^{00}, \boldsymbol{\mu}^{00} \boldsymbol{\mu}^{0 u} \boldsymbol{\mu}^{u 0}, \boldsymbol{\mu}^{0 u} \boldsymbol{\mu}^{u 0} \boldsymbol{\mu}^{00}, \boldsymbol{\mu}^{0 u} \boldsymbol{\mu}^{u u} \boldsymbol{\mu}^{u 0}$, respectively. From the three time orderings we therefore have $3 \times 2^{2}=12$ contributions-each a product of three 'transition' dipoles (one or more of which may be permanent), divided by a product of two energy factors. Application of the algorithm determines that only the state sequence $0 u u 0\left(\boldsymbol{\mu}^{0 u} \boldsymbol{\mu}^{u u} \boldsymbol{\mu}^{u 0}\right)$ need be considered in a suitable reinterpretation of the three time-ordered diagrams, since each of the other possibilities generates a $\boldsymbol{\mu}^{00}$ segment. Utilizing Fig. 2, we therefore obtain a two-level hyperpolarizability tensor exactly as expressed by Eq. (85). This is the simplest example of how the algorithm quickly generates results that would otherwise demand considerable algebraic manipulation. However, it is with higher-order amplitudes that the method is most obviously efficacious. Even in (85), the tensor structure obviates simple factorization in terms of $\breve{\mathbf{d}}$; higher orders have the additional complication that terms both linear and in powers of $\breve{\text { d }}$ arise. 
The process of third-harmonic generation (THG) serves both to illustrate the power of the new algorithm and to draw out some new physics. To derive the form of the susceptibility tensor, one needs to employ either the four time-ordered diagrams or the equivalent state-sequence diagram representing THG. Both diagrammatic representations are illustrated in Fig. 9. The state route connecting the initial and final (ground) states here runs through three virtual states, $r, s$, and $t$, and the two-level approximation requires each to be either the ground or the excited state. In this case, from the four time orderings we get a total of $4 \times 2^{3}=32$ contributions, each a product of four transition or permanent dipoles divided by three energy quotients. With the benefit of the algorithmic method delineated above, we can take the four time orderings and dispense with all except two of the following state sequences: $00000,000 u 0,00 u 00,00 u u 0,0 u 000,0 u 0 u 0,0 u u 00,0 u и u 0$. Specifically, discarding each sequence that includes the segment 00 , we retain only $0 u 0 u 0$ and $0 u и u 0$. With proper reinterpretation of these remaining cases, we thus immediately obtain the following explicit result comprising only eight terms, of which each successive pair results from the successive time-ordered diagrams of Fig. 9a, also corresponding to all routes through the state-sequence

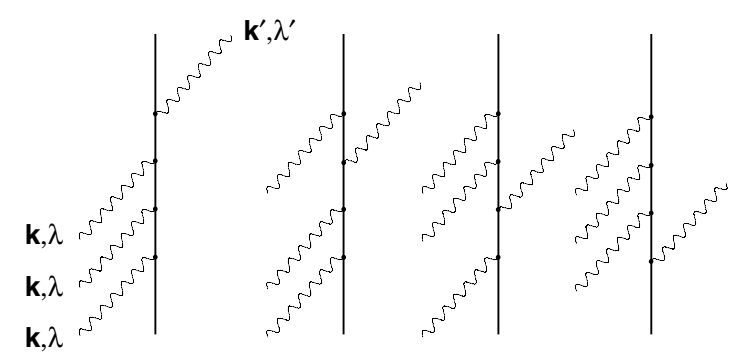

(a)

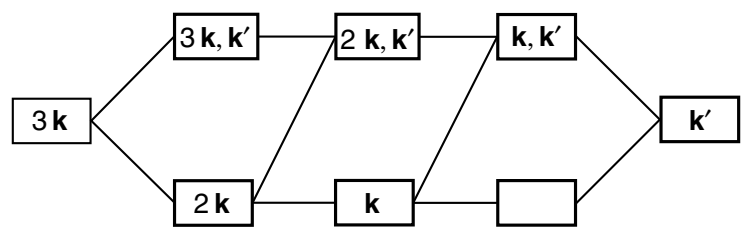

(b)

Figure 9. The four time-ordered diagrams characterizing third harmonic generation (a) and the state-sequence diagram representing the same process $(b)$. 
diagram of Fig. 9b. [80]

$$
\begin{aligned}
\gamma_{i j k l}(-3 \omega ; \omega, \omega, \omega)= & \frac{\mu_{i}^{u 0} \mu_{j}^{u 0} \mu_{k}^{u 0} \mu_{l}^{u 0}}{\left(E_{u 0}-3 \hbar \omega-i \Gamma_{u}\right)(-2 \hbar \omega)\left(E_{u 0}-\hbar \omega-i \Gamma_{u}\right)} \\
& +\frac{\mu_{i_{1}}^{u 0} \breve{d}_{j} \breve{d}_{k} \mu_{l}^{u 0}}{\left(E_{u 0}-3 \hbar \omega-i \Gamma_{u}\right)\left(E_{u 0}-2 \hbar \omega-i \Gamma_{u}\right)\left(E_{u 0}-\hbar \omega-i \Gamma_{u}\right)} \\
& +\frac{\mu_{j}^{u 0} \mu_{i}^{u 0} \mu_{k}^{u 0} \mu_{l}^{u 0}}{\left(E_{u 0}+\hbar \omega-i \Gamma_{u}\right)(-2 \hbar \omega)\left(E_{u 0}-\hbar \omega-i \Gamma_{u}\right)} \\
& +\frac{\mu_{j}^{u 0} \breve{d}_{i} \breve{d}_{k} \mu_{l}^{u 0}}{\left(E_{u 0}+\hbar \omega-i \Gamma_{u}\right)\left(E_{u 0}-2 \hbar \omega-i \Gamma_{u}\right)\left(E_{u 0}-\hbar \omega-i \Gamma_{u}\right)} \\
& +\frac{\mu_{j}^{u 0} \mu_{k}^{u 0} \mu_{i}^{u 0} \mu_{l}^{u 0}}{\left(E_{u 0}+\hbar \omega-i \Gamma_{u}\right)(2 \hbar \omega)\left(E_{u 0}-\hbar \omega-i \Gamma_{u}\right)} \\
& +\frac{\mu_{j}^{u 0} \breve{d}_{k} \breve{d}_{i} \mu_{l}^{u 0}}{\left(E_{u 0}+\hbar \omega-i \Gamma_{u}\right)\left(E_{u 0}+2 \hbar \omega-i \Gamma_{u}\right)\left(E_{u 0}-\hbar \omega-i \Gamma_{u}\right)} \\
& +\frac{\mu_{j}^{u 0} \mu_{k}^{u 0} \mu_{l}^{u 0} \mu_{i}^{u 0}}{\left(E_{u 0}+\hbar \omega-i \Gamma_{u}\right)(2 \hbar \omega)\left(E_{u 0}+3 \hbar \omega-i \Gamma_{u}\right)} \\
& +\frac{\mu_{j}^{u l} \breve{d}_{k} \breve{d}_{l} \mu_{i}^{u 0}}{\left(E_{u 0}+\hbar \omega-i \Gamma_{u}\right)\left(E_{u 0}+2 \hbar \omega-i \Gamma_{u}\right)\left(E_{u 0}+3 \hbar \omega-i \Gamma_{u}\right)}
\end{aligned}
$$

where once again it is the index-symmetrized form, here entailing all permutations $(j k l)$, that will feature in the observables. The transition moment $\boldsymbol{\mu}^{u 0}$ is taken to be real and hence equal to $\boldsymbol{\mu}^{0 u}$; also note that the tensor $\gamma$ is minus that given as $\gamma^{00}(-3 \omega ; \omega, \omega, \omega)$ elsewhere [80], because here each energy denominator expression carries an overall a minus sign, for consistency within the current work.

One aspect of Eq. (90) deserving comment is its amenability for the identification of resonances. Three-photon resonances are manifest in the first and second terms, through the appearance of the factor $\left(E_{u 0}-3 \hbar \omega-i \Gamma_{u}\right)$; twophoton resonances $\left(E_{u 0}-2 \hbar \omega-i \Gamma_{u}\right)$ are featured in the second and fourth, and single-photon resonances $\left(E_{u 0}-\hbar \omega-i \Gamma_{u}\right)$ are seen in each of the first six. Since exploitation of the latter kind of resonance is in practice usually avoided because of the competing linear absorption with which it is associated, it is the two- and three- photon resonances that are of the most interest. Under suitable conditions, third-harmonic generation in either of those cases is driven largely by just two of the contributions to Eq. (90). Other contributions, signifying 
minor corrections, are of much the same order of magnitude as those relating to the involvement of other molecular energy levels.

Further features are evident when the relative magnitudes of the dipole difference $|\mathbf{d}|$ and the transition dipole $\left|\boldsymbol{\mu}^{u 0}\right|$ are considered. One immediately striking feature is the observation that the second, fourth, sixth, and eighth terms all disappear if $|\mathbf{d}|=0$, leaving only terms associated with virtual excitation routes. [Note that no such routes were manifest in the second-harmonic result. If $|\mathbf{d}|=0$ then the entire expression Eq. (85) becomes zero-any process involving an odd number of photons has to entail at least one 00 or $u и$ segment in the interaction sequence.] In the third-harmonic case, in particular, both terms associated with two-photon resonances disappear-in other words, there can be no two-photon resonance enhancement of third-harmonic generation under such circumstances. If, however, $|\mathbf{d}| \gg\left|\boldsymbol{\mu}^{u 0}\right|$, then the even terms of Eq. (90) dominate the optical response-and in the case of three-photon resonance, it is the second term that provides by far the leading contribution. Such considerations should play an important role in implementing strategies for the calculation of nonlinear optical response; for example in the case just cited, the dominant term is of a form that had not previously been identified as representing the major contribution.

\section{OPTICAL COHERENCE IN DISPERSED PARTICLES}

Despite the powerful symmetry rule that precludes the generation of even harmonics in optically isotropic media, except at surfaces, a number of experimental results have indicated exceptions to the rule, as detailed in the earlier review [1]. Most entail conditions resulting in a transient, local removal of isotropy, and are therefore well understood. Nonetheless, two quite different mechanisms have been found to mediate second-harmonic generation in macroscopically isotropic systems. In this section we consider a mechanism relating to optical coherence in small particles in suspension, or locally ordered domains within macroscopically structureless media. In the next section we shall focus on a six-wave form of interaction associated with very high pump laser intensities.

The coherent generation of second harmonics from particles in suspension was first found to operate in the generation of strongly directed SHG signals from photosynthetic bacterial membranes, randomly oriented in aqueous suspension $[82,83]$. The paradox was resolved when it was shown that the unusually strong signal detected from such isotropic suspensions is attributable to optical coherence within the separate particles of the suspension [84]. As such, the harmonic emission displays an amalgam of the characteristics associated with full coherence (second-harmonic generation) and incoherence (hyper-Rayleigh scattering). To understand this, it is necessary to return to the development of theory in Section VII. Consider a fluid or mesoscopically disordered material 
(or any other optically isotropic system) within which there are small particles or local domains possessing a microscopically ordered structure. Examples of such systems include colloids, cell and membrane suspensions, and many plastics, glasses, and other modern materials. In systems of interest, each particle or domain includes a significant number of optical centers with strong optical dispersion at the frequency of the pump laser radiation or its harmonic. Let us suppose that such a system contains $M$ randomly oriented particles (individually denoted below by the subscript $m$ ), each composed of $n$ discrete molecules or other optical centres (denoted by the subscript $\xi$ ). Within each particle it is assumed that there is a structurally imposed orientational correlation, that is, a significant intrinsic rigidity, such that its net optical response can be cast in terms of an "effective hyperpolarizability" tensor given by

$$
\beta_{(m) i j k}^{00}=\sum_{\xi}^{n} \beta_{(\xi) \lambda \mu v}^{00} l_{i \lambda}^{\xi} l_{j \mu}^{\xi} l_{k v}^{\xi} e^{i \Delta \mathbf{k} \cdot\left(\mathbf{R}_{\xi}-\mathbf{R}_{m}\right)}
$$

Here the hyperpolarizability of each individual optical center, $\beta_{(\xi) \lambda \mu v}^{00}$, is given a superscript label 00 to indicate that it relates to the electronic ground state (an assumption to be revisited later), and its position is given relative to the common Cartesian frame. The factor $l_{i \lambda}^{\xi}\left(l_{j \mu}^{\xi}, l_{k v}^{\xi}\right)$ is the cosine of the angle between the space fixed axis $i(j, k)$ and the molecule fixed axis $\lambda(\mu, v)$. If the particles or ordered domains are small compared to the optical wavelengths involved, then so will be the internal distances $\left(\mathbf{R}_{\xi}-\mathbf{R}_{m}\right)$, and thus in many circumstances-even in the complete lack of wavevector matching $(\Delta \mathbf{k}=0)$ - the phase factor in Eq. (91) can often be taken as effectively unity. However, we retain its explicit form for generality.

The rate of second-harmonic generation by the entire system of particles is expressible as a sum of two terms $\Gamma_{1}$ and $\Gamma_{2}$ as follows, where angular brackets denote the orientational average

$$
\begin{aligned}
& \Gamma_{1}=M \varsigma\left\langle\left|\beta_{(m) i j k}^{00} \bar{e}_{i}^{\prime} e_{j} e_{k}\right|^{2}\right\rangle \\
& \Gamma_{2}=\left(\eta_{M}-M\right) \varsigma\left|\left\langle\beta_{(m) i j k}^{00} \bar{e}_{i}^{\prime} e_{j} e_{k}\right\rangle\right|^{2}
\end{aligned}
$$

in which the latter, which represents a coherent addition of SHG amplitudes from every optical center in the system, corresponds exactly to the earlier Eq. (59) (which thereby serves to define the parameter $\zeta$ ). When the particles are randomly oriented, $\Gamma_{2}$ vanishes as a result of the isotropic average, as is well known. However, since the corresponding average is conducted over the modulus square in the "incoherent" term, $\Gamma_{1}$, this contribution persists, representing an addition of the harmonic intensities produced by different particles. As determined by Eq. (91), these contributions in fact accommodate a coherent 
addition of signals from the various optical centers that each particle contains. The analysis of angularly resolved measurements of the second harmonic [85] led to experimental verification of this interpretation, as shown in Fig. 10.

The coherent addition of second-harmonic signals, which can occur only in regions of local order, leads to intriguing possibilities for materials strongly pumped by an ultrafast source [86]. Here, the key feature is the relationship between the hyperpolarizabilities of optical centers in their ground and electronic excited states, under resonance conditions. To investigate this further, we return to the two-level model of the previous section, considering the role of other electronic levels subsequently. The hyperpolarizability for the upper state $u$ is readily obtained using the transformed interaction Hamiltonian:

$$
H_{\text {int }}^{\prime \prime}=-\varepsilon_{0}^{-1}\left\lfloor\boldsymbol{\mu}-\boldsymbol{\mu}^{u u}\right\rfloor \cdot \mathbf{e}^{\perp}
$$

Here the algorithm given in (89) is modified by interchanging the labels 0 and $u$ :

$$
\boldsymbol{\mu}^{00} \rightarrow \boldsymbol{\mu}^{00}-\boldsymbol{\mu}^{u u}=-\mathbf{d} ; \quad \boldsymbol{\mu}^{u u} \rightarrow 0
$$

This has the effect of reversing the sign of $\mathbf{d}$ and also the energy difference $E_{u 0}$, wherever each appears, although the Hermiticity of the dipole operator ensures that for nondegenerate states the transition dipole suffers no change:

$$
\begin{aligned}
\mathbf{d} & =\boldsymbol{\mu}^{u u}-\boldsymbol{\mu}^{00} \rightarrow-\mathbf{d}=-\left(\boldsymbol{\mu}^{u u}-\boldsymbol{\mu}^{00}\right) \\
E_{u 0} & =E_{u}-E_{0} \rightarrow-E_{u 0}=E_{0 u} \\
\boldsymbol{\mu}^{0 u} & =\boldsymbol{\mu}^{u 0}
\end{aligned}
$$

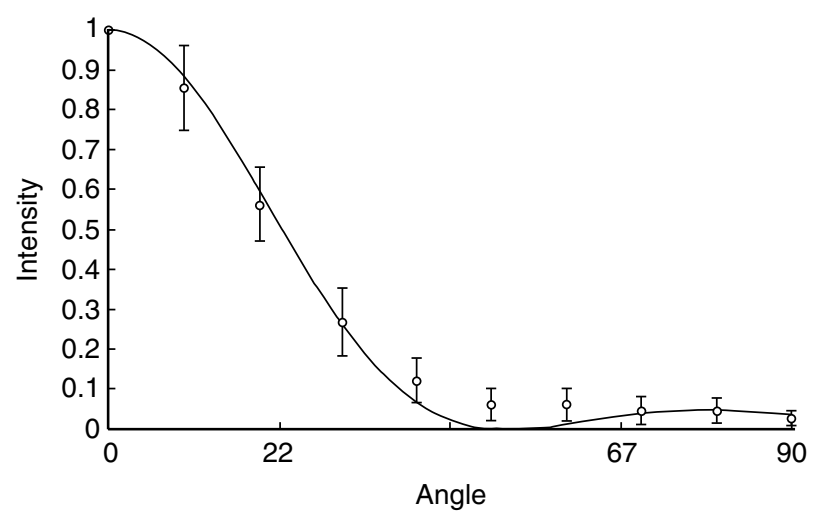

Figure 10. Intensity (arbitrary units) of optical SHG from a purple membrane suspension. Experimental data (Allcock et al. 1996) obtained with Nd:YAG laser pumping are shown by the open circles and the solid line represents the theoretical fit. 
It then transpires that the upper-level hyperpolarizability tensor stands in the following simple relationship to its ground-state counterpart [86]:

$$
\beta_{i j k}^{00}(-2 \omega, \omega, \omega)=-\beta_{i j k}^{u u}(-2 \omega, \omega, \omega)
$$

Similar features arise when third-harmonic generation is considered. In passing we note that a variable-sign convention for the damping would lead to behavior of an analytical form substantially different from that discussed below.

Consider a system in which, prior to the input of the pump radiation responsible for the detected harmonic output, irradiation with a beam of the appropriate resonant frequency produces a significant population of the electronic level $u$ among the optical centers in each particle or domain. With firstorder decay kinetics, the probability that a certain center $\xi$ is excited at time $t$ is given by

$$
P\left(\mathbf{R}_{\xi}, t\right)=P_{0}\left(\mathbf{R}_{\xi}\right) \exp \left(-k\left(t-t_{0}\right)\right)
$$

where $k$ is the decay constant and $P_{0}$ is the residual probability that the upper level is excited at time $t_{0}$, the time at which the pump radiation for SHG detection is applied. Particular interest expressed below focuses on the case of ultrafast excitation of sufficient intensity to elicit the onset of saturation, where $P_{0}>0.5$. While both excited- and ground-state species are present, the two processes described above contribute to an effective unit hyperpolarizability given by

$$
\begin{aligned}
\beta_{(m) i j k} & =\sum_{\xi}^{n}\left[\left(1-P\left(\mathbf{R}_{\xi}, t\right)\right) \beta_{(\xi) \lambda \mu v}^{00}+P\left(\mathbf{R}_{\xi}, t\right) \beta_{(\xi) \lambda \mu v}^{u u}\right] l_{i \lambda}^{\xi} l_{j \mu}^{\xi} k v^{\xi} e^{i \Delta \mathbf{k} \cdot\left(\mathbf{R}_{\xi}-\mathbf{R}_{m}\right)} \\
& =\sum_{\xi}^{n}\left[\left(1-2 P\left(\mathbf{R}_{\xi}, t\right)\right) \beta_{(\xi) \lambda \mu v}^{00}\right] l_{i \lambda}^{\xi} l_{j \mu}^{\xi} l_{k v}^{\xi} e^{i \Delta \mathbf{k} \cdot\left(\mathbf{R}_{\xi}-\mathbf{R}_{m}\right)}
\end{aligned}
$$

leading to a harmonic intensity that features a characteristic decay and recovery in its temporal profile. Let us assume for simplicity that the probability of initial excitation is identical for all optical centers, removing the $\mathbf{R}_{\xi}$ dependence of $P\left(\mathbf{R}_{\xi}, t\right)$. Let us also denote by $\boldsymbol{\beta}$ the key factor

$$
\boldsymbol{\beta}=\sum_{\xi}^{n} \beta_{(\xi) \lambda \mu \nu}^{00} l_{i \lambda}^{\xi} l_{j \mu}^{\xi} l_{k v}^{\xi} \bar{e}_{i}^{\prime} e_{j} e_{k} e^{i \Delta \mathbf{k} \cdot\left(\mathbf{R}_{\xi}-\mathbf{R}_{m}\right)}
$$

which will in general be a complex quantity by virtue of the damping involved in the hyperpolarizability tensor. For simplicity, assuming continuous-wave pump 
radiation, the intensity of harmonic emission thus acquires a time dependence of the biexponential form

$$
I \sim|\boldsymbol{\beta}|^{2}\left[1-4 \exp \left(-k t^{\prime}\right)+4 \exp \left(-2 k t^{\prime}\right)\right]
$$

where $t^{\prime}=t-t_{0}$ and $P_{0}\left(\mathbf{R}_{\xi}\right) \equiv 1$, giving a trace of the form $G(t)=1-4$ $\exp (-k t)+4 \exp (-2 k t)$ as shown in Fig. 11.

To observe this exact time dependence in the second harmonic would require the satisfaction of certain criteria detailed below. Nonetheless, these conditions are largely a reflection of the simple two-level model employed, and in the following discussion we show that the major features of the result should be manifest in real systems of considerably greater electronic complexity. First, we note that with the two-level model a necessary condition for observation of a fall, and recovery of the harmonic output as illustrated in Fig. 11 is the creation of a transient population inversion by the preceding excitation laser pulse. The minimum output intensity (which will, in fact, be nonzero) would then be obtained at the time where the fractional population of the upper level $u$ has fallen to exactly 0.5 , matching the ground-state population in a two-level system. In practice, achieving initial population inversion is likely to require that the excitation pulse populate a higher level $h$ that rapidly decays to $u$, where the latter plays the role of a population bottleneck as in conventional laser action. Although this emphasizes the fact that a two-level representation of the

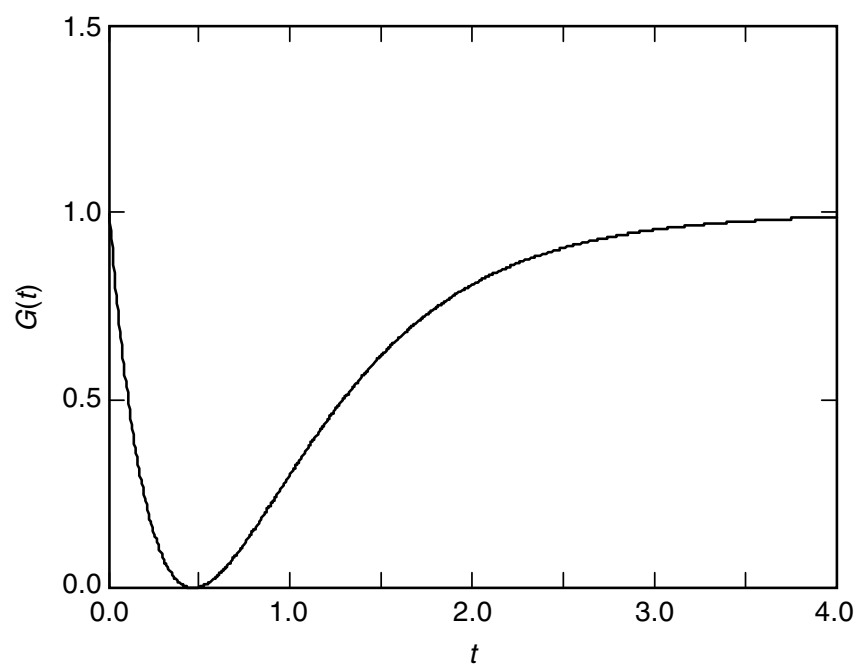

Figure 11. Schematic of the harmonic temporal profile $G(t)$ from Eq. (100) in arbitrary units. 
electronic energy levels is necessarily incomplete—as, indeed, it generally isthe inclusion of $h$ and any other levels in the electronic structure of the optical centers will not significantly affect the results, provided those levels are no longer significantly populated once the pump for harmonic emission is applied. The harmonic signal will still be dominated by generation in centers in either the 0 or $u$ state. Other electronic levels will certainly play the role of virtual states in the hyperpolarizabilities of each of those levels, adding a background contribution to each.

The effect of involving other levels in the calculations is assessed as follows, by considering what modifications to the preceding theory ensue. Both the tensors $\boldsymbol{\beta}^{00}$ and $\boldsymbol{\beta}^{u u}$ certainly acquire additional (and different) background contri butions, also complex, such that

$$
\left.\begin{array}{c}
\sum_{\xi}^{n} \beta_{(\xi) \lambda \mu v}^{00} l_{i \lambda}^{\xi} l_{j \mu}^{\xi} l_{k v}^{\xi} \bar{e}_{i}^{\prime} e_{j} e_{k} e^{i \Delta \mathbf{k} \cdot\left(\mathbf{R}_{\xi}-\mathbf{R}_{m}\right)} \rightarrow \boldsymbol{\beta}+\tilde{\boldsymbol{\beta}} \\
\sum_{\xi}^{n} \beta_{(\xi) \lambda \mu v}^{u u} l_{i \lambda}^{\xi} l_{j \mu}^{\xi} l_{k v}^{\xi} \bar{e}_{i}^{\prime} e_{j} e_{k} e^{i \Delta \mathbf{k} \cdot\left(\mathbf{R}_{\xi}-\mathbf{R}_{m}\right)} \rightarrow-\boldsymbol{\beta}+\check{\boldsymbol{\beta}}
\end{array}\right\}
$$

This results in a harmonic intensity with a more intricate time dependence of the form

$$
I \sim\left|(\boldsymbol{\beta}+\tilde{\boldsymbol{\beta}})-(2 \boldsymbol{\beta}+\tilde{\boldsymbol{\beta}}-\breve{\boldsymbol{\beta}}) \exp \left(-k t^{\prime}\right)\right|^{2}
$$

which no longer factorises out the temporal profile $G\left(t^{\prime}\right)$. Separating the real and imaginary parts of each parameter $\boldsymbol{\beta}=\boldsymbol{\beta}^{\prime}+i \boldsymbol{\beta}^{\prime \prime}, \tilde{\boldsymbol{\beta}}=\tilde{\boldsymbol{\beta}}^{\prime}+i \widetilde{\boldsymbol{\beta}}^{\prime \prime}, \breve{\boldsymbol{\beta}}=\breve{\boldsymbol{\beta}}^{\prime}+i \breve{\boldsymbol{\beta}}^{\prime \prime}$, we thus have

$$
\begin{aligned}
I \sim & {\left[\left(\boldsymbol{\beta}^{\prime}+\tilde{\boldsymbol{\beta}}^{\prime}\right)-\left(2 \boldsymbol{\beta}^{\prime}+\tilde{\boldsymbol{\beta}}^{\prime}-\breve{\boldsymbol{\beta}}^{\prime}\right) \exp \left(-k t^{\prime}\right)\right]^{2}+\left[\left(\boldsymbol{\beta}^{\prime \prime}+\tilde{\boldsymbol{\beta}}^{\prime \prime}\right)\right.} \\
& \left.-\left(2 \boldsymbol{\beta}^{\prime \prime}+\tilde{\boldsymbol{\beta}}^{\prime \prime} \breve{\boldsymbol{\beta}}^{\prime \prime}\right) \exp \left(-k t^{\prime}\right)\right]^{2}
\end{aligned}
$$

Of the two intensity contributions in Eq. (103) the first, associated with the real parts of the hyperpolarizabilities, will generally dominate, leading to a minimum in the harmonic emission at a time given by

$$
t_{\min }^{\prime} \sim k^{-1} \ln \left(\frac{2 \boldsymbol{\beta}^{\prime}+\tilde{\boldsymbol{\beta}}^{\prime}-\breve{\boldsymbol{\beta}}^{\prime}}{\boldsymbol{\beta}^{\prime}+\tilde{\boldsymbol{\beta}}^{\prime}}\right)
$$

However, the effect of the second contribution in Eq. (103), corresponding to the imaginary parts of the hyperpolarizabilities and due to damping effects, will be to obviate complete cancellation of the harmonic signal at this time-only by a 
spurious accident of hyperpolarizability values could the two harmonic intensity contributions of Eq. (103) be simultaneously zero. The characteristic fall and recovery of the harmonic remains.

In the light of the results presented above, it is useful to recall that the behavior we have identified, in the second-harmonic profile of a system of randomly oriented small particles or ordered domains in complex materials, owes its origin to the local quantum coherence between harmonic emission processes at different optical centers within each particle or domain. This behavior is dominated by features associated with a two-level optical response, but, provided the pump or harmonic frequencies are close to resonance, broadly similar effects are anticipated in systems of considerably greater electronic complexity. The effects of damping, which have to be included during operation close to resonance, and also the effects (as virtual states) of higher electronic levels, are to produce a background emission that prevents the harmonic from falling quite to zero during the probe pulse throughput. The characteristic signal recovery nonetheless remains a key feature, and its detailed form reflects the correct (constant sign) convention for effecting the optical damping. In this sense, observations might provide ground for experimental verification of the signing. Determination of the biexponential form of the harmonic profile will faithfully register the dynamics of excited-state decay.

The result has structural as well as kinetic implications. The local coherence, responsible for the partial cancellation of the harmonic signal after a characteristic delay time, is entirely dependent on a structural rigidity within each particle. This need not mean that all the optical centers are identically aligned, but that they do not rotate significantly with respect to each other (at least over the timescale for the harmonic measurements). The extent of recovery in the harmonic signal serves to register the extent of local coherence, and hence in many systems the localization of structural order. In any less than completely rigid system, it might be possible to assess the degree of local flexibility from the extent of harmonic recovery.

\section{SIX-WAVE SECOND HARMONIC GENERATION}

A spate of papers since the mid-1990s have reported theoretical and experimental studies of second-harmonic generation mediated by six-wave mixing (SWM), reflecting the new availability of laser sources with sufficient power and stability to make such observations possible [87-95]. Here we review the theory underlying the six-wave mechanisms for the evolution of a coherent secondharmonic signal in media where it is normally forbidden. As we shall see, the process $\omega+\omega+\omega+\omega \rightarrow 2 \omega+2 \omega$ is invariably permitted, irrespective of local or bulk symmetry. We show how the initial results can be neatly adapted to model real molecular systems. We conclude by exhibiting the experimentally verified 
form of a time-delayed harmonic that emerges if a pump probe system of beams is employed, which once again is a manifestation of quantum-optical interference.

To calculate the rate, we first construct a matrix element using the general equation (41) and substitute into the Golden Rule, Eq. (51). The detailed structure of the nonlinear polarizability $\boldsymbol{\alpha}_{\mathrm{SWM}}^{(6)}(-2 \omega,-2 \omega ; \omega, \omega, \omega, \omega)$, which the process entails, is obtained using the state-sequence diagram of Fig. 12; in all there are $15(=6 ! / 4 ! 2 !)$ pathways linking the initial and final states to be taken into consideration, representing all topologically distinct orderings of the six electric dipole interactions involved. Again we might have used the timeordered diagrams as an alternative-both, of course, lead to identical tensor expressions-but the concise representation of the state-sequencing is now clearly evident. For the explicit representation of the somewhat unwieldy resulting expressions, it is convenient to employ a more compact notation than we have used for lower-order polarisabilities. Here we follow Naguleswaran and Stedman [96], and neatly express $\alpha_{\mathrm{SWM}}^{(6)}\left(-\omega^{\prime},-\omega^{\prime} ; \omega, \omega, \omega, \omega\right)$ as

$$
\begin{aligned}
\alpha_{\mathrm{SWM}}^{(6)}\left(-\omega^{\prime},-\omega^{\prime} ; \omega, \omega, \omega, \omega\right)= & \sum_{\pi} \sum_{r, s, t, u, v}\left\{\left(\mu_{\pi(6)}^{0 v} \mu_{\pi(5)}^{v u} \mu_{\pi(4)}^{u t} \mu_{\pi(3)}^{t s} \mu_{\pi(2)}^{s r} \mu_{\pi(1)}^{r 0}\right)\right. \\
& {\left[\{ \tilde { E } _ { r 0 } + \hbar \eta _ { \pi ( 1 ) } \omega _ { \pi ( 1 ) } \} \left\{\tilde{E}_{s 0}+\hbar\left(\eta_{\pi(1)} \omega_{\pi(1)}+\eta_{\pi(2)} \omega_{\pi(2)}\right)\right.\right.} \\
& \times\left\{\tilde{E}_{t 0}+\hbar\left(\eta_{\pi(1)} \omega_{\pi(1)}+\eta_{\pi(2)} \omega_{\pi(2)}+\eta_{\pi(3)} \omega_{\pi(3)}\right)\right\} \\
& \times\left\{\tilde{E}_{u 0}+\hbar\left(\eta_{\pi(1)} \omega_{\pi(1)}+\eta_{\pi(2)} \omega_{\pi(2)}+\eta_{\pi(3)} \omega_{\pi(3)}\right.\right. \\
& \left.+\eta_{\pi(4)} \omega_{\pi(4)}\right) \times\left\{\tilde{E}_{v 0}+\hbar\left(\eta_{\pi(1)} \omega_{\pi(1)}\right.\right. \\
& \left.\left.\left.+\eta_{\pi(2)} \omega_{\pi(2)}+\eta_{\pi(3)} \omega_{\pi(3)}+\eta_{\pi(4)} \omega_{\pi(4)} \eta_{\pi(5)} \omega_{\pi(5)}\right)\right\}\right]^{-1}
\end{aligned}
$$

where the sign of the photon label $\eta_{\pi(n)}=+1$ or -1 for emission or absorption, respectively. The sum over $\pi$ leads to 30 unique permutations, allowing for

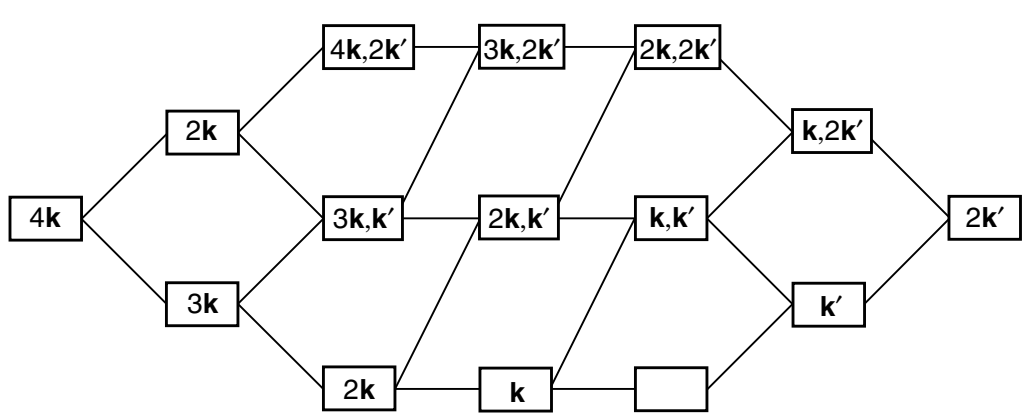

Figure 12. The state-sequence diagram representing SWM. 
reversal of the time orderings of the signal photons, as is necessary for harmonics produced with differing wavevectors or polarizations. As an example of the energy denominator for a particular time ordering, consider the term for which $\pi(1)=n, \pi(2)=m, \pi(3)=l, \pi(4)=k, \pi(5)=j$, and $\pi(6)=i$. An energy denominator of the following form emerges

$\left[\left(\tilde{E}_{r 0}-\hbar \omega\right)\left(\tilde{E}_{s 0}-2 \hbar \omega\right)\left(\tilde{E}_{t 0}-2 \hbar \omega+\hbar \omega^{\prime}\right)\left(\tilde{E}_{u 0}-3 \hbar \omega+\hbar \omega^{\prime}\right) r\left(\tilde{E}_{v 0}-4 \hbar \omega+\hbar \omega^{\prime}\right)\right]$

corresponding to the following temporal ordering: absorptions $n$ and $m(\omega)$, emission $l\left(\omega^{\prime}\right)$, absorptions $k$ and $j(\omega)$ and finally emission $i\left(\omega^{\prime}\right)$.

It is worth drawing attention to the presence, and means of dealing with, the secular resonances that arise in high orders of optical nonlinearity. These features represent the fact that the quantum amplitudes for such processes can subsume the matrix elements for lower-order processes, apparently leading to spurious infinities. The various time orderings of the SWM interaction described here clearly include some that factor as a product of two sequential SHG time orderings, and it repays effort to examine in more detail their structure in the SWM polarizability tensor. Consider a situation where the molecular intermediate state $|t\rangle$ is represented by the molecular ground state $|0\rangle$ in the sum over $t$. When this occurs, certain energy denominators, such as $E_{t i}$, can suffer a complete cancellation of the radiation terms to uncover an expression of the form $E_{00}-i \Gamma_{0}=-i \Gamma_{0}$; see, for example, Table III, which lists the energy denominators. As the ground-state lifetime, represented by $\Gamma_{0}^{-1}$, is considered infinite, a divergent signal is suggested. In order to circumvent these secular resonances, the molecular polarizability has to be reconstructed in such a way as to remove the possibility of infinite response [79]. We have reported the details for such a reconstruction explicitly for the SWM polarizability tensor [91]. The procedure is straightforward and entails properly taking the limit as the virtual state energy approaches that of the ground state.

Returning to the general form of the SWM interaction, it is next necessary to form the radiation tensor using the general expression of Eq. (52). Explicitly incorporating the degree of coherence of the input beam and assuming that there are initially no photons in the harmonic mode, the following equation, where $I \omega$ is the intensity of the pump radiation, is obtained for the rate of six-wave mixing:

$$
\begin{aligned}
\Gamma_{\mathrm{SWM}}= & \left(\frac{4 \pi \hbar}{\varepsilon_{0}^{6}}\right)\left(\frac{I_{\omega}}{2 c n_{\omega}}\right)^{4}\left(\frac{n_{\omega}+2}{3}\right)^{8} \sum_{\mathbf{k}^{\prime}, \lambda^{\prime}}\left(\frac{v_{g}^{\prime} \omega^{\prime}}{2 c V n_{\omega^{\prime}}}\right)^{2}\left(\frac{n_{\omega^{\prime}}+2}{3}\right)^{4} g_{\omega}^{(4)} \eta_{N} \\
& \times\left|\left\langle\alpha^{(6)}(-2 \omega,-2 \omega ; \omega, \omega, \omega, \omega) \cdot \overline{\mathbf{e}}^{\prime} \overline{\mathbf{e}}^{\prime} \mathbf{e e e e}\right\rangle\right|^{2} \delta\left(2 \hbar \omega_{k^{\prime}}-4 \hbar \omega_{k}\right)
\end{aligned}
$$

The result embodies the coherence factor $\eta_{N}$ to account for the phase-matching characteristics of the process, leading to the familiar $\operatorname{sinc}^{2}$ behavior, which 


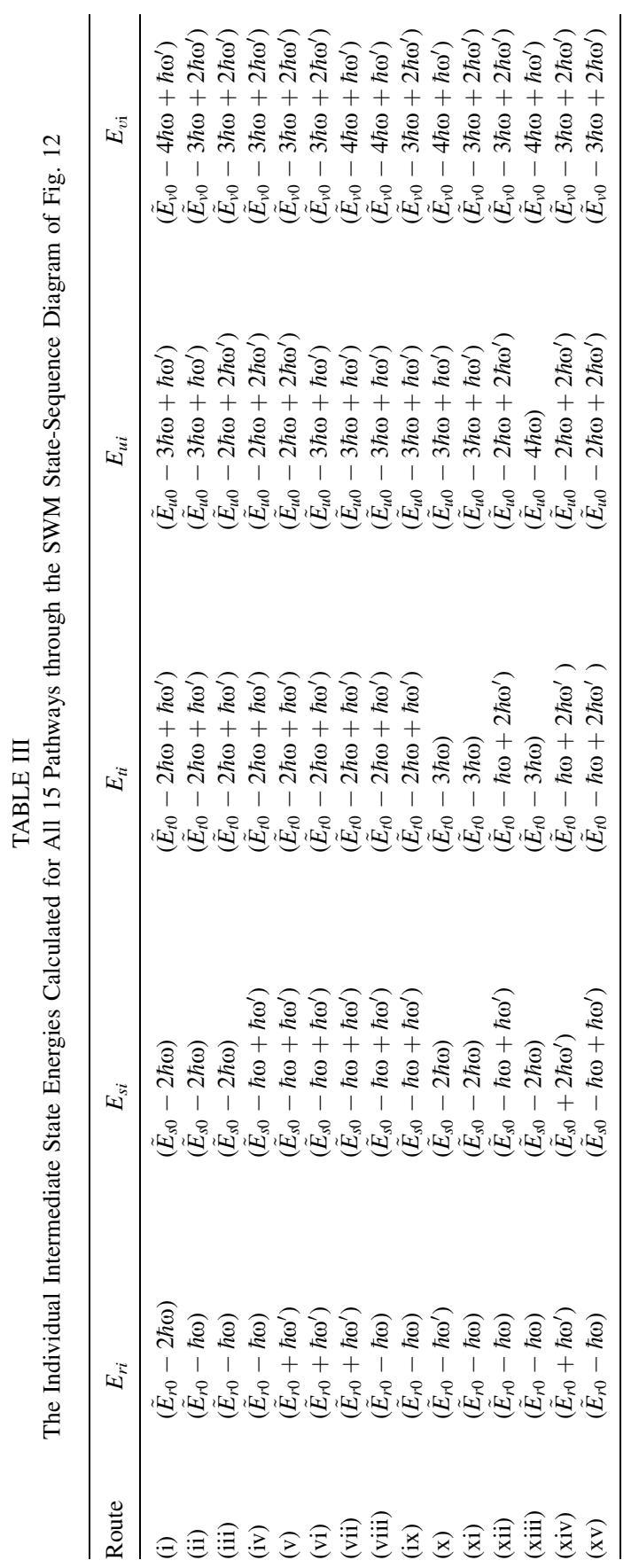


demonstrates that the SWM process manifests coherence for emission in the forward $z$ direction. This is nonetheless subject to optimal wavevector matching: $\Delta \mathbf{k}=4 \mathbf{k}-\mathbf{k}^{\prime} \approx 0$.

The question of whether SWM can generate an observable signal in fluids requires further analysis, and calls for explicit evaluation of the ensemble average of the tensor product in Eq. (106) as

$$
\left\langle\boldsymbol{\alpha}^{(6)} \cdot \overline{\mathbf{e}}^{\prime} \overline{\mathbf{e}}^{\prime} \mathbf{e e e e}\right\rangle=\alpha_{(\lambda \mu)(v o \pi \rho)}^{(6)} \bar{e}_{i}^{\prime} \bar{e}_{j}^{\prime} e_{k} e_{l} e_{m} e_{n}\left\langle\ell_{i \lambda} \ell_{j \mu} \ell_{k v} \ell_{l o} \ell_{m \pi} \ell_{n \rho}\right\rangle
$$

Applying a sixth-rank rotational average [97] immediately reveals that the rate equation entails an overall multiplier of the scalar product (e $\cdot \mathbf{e})$, which vanishes for circular polarizations. The six-wave interaction is thus subject to the same embargo on conversion of a circularly polarized pump as the conventional SHG process [98]. In the case of a plane-polarized pump, ensemble averaging leads to the result;

$$
\begin{aligned}
\Gamma_{\mathrm{SWM}}= & \left(\frac{4 \pi \hbar \omega_{k}^{2}}{\varepsilon_{0}^{6}}\right)\left(\frac{I_{\omega}}{2 c n_{\omega}}\right)^{4}\left(\frac{n_{\omega_{k}}^{2}+2}{3}\right)^{8} g_{\omega}^{(4)} \sum_{\mathbf{k}^{\prime}, \lambda^{\prime}}\left(\frac{v_{g}^{\prime} \omega_{k^{\prime}}^{\prime}}{2 V c n \omega_{k^{\prime}}^{\prime}}\right)^{2}\left(\frac{n_{\omega_{k^{\prime}}^{\prime}}^{2}+2}{3}\right)^{4} \\
& \times\left|\left\{6\left(\mathbf{e} \cdot \mathbf{e}^{\prime}\right)^{2}-2\right\} \alpha_{(\lambda \mu)(\lambda \mu v v)}^{(6)}-\left\{2\left(\mathbf{e} \cdot \mathbf{e}^{\prime}\right)^{2}-3\right\} \alpha_{(\lambda \lambda)(\mu \mu v v)}^{(6)}\right|^{2} \eta_{N} \\
& \times \delta\left(2 \hbar \omega_{k^{\prime}}^{\prime}-4 \hbar \omega_{k}\right)
\end{aligned}
$$

following simplication exploiting the inherent permutational symmetry in the first two and last four indices of the nonlinear response tensor. Equation (108) illustrates the fact that there need not be full retention of polarization in the emitted harmonic; indeed, the extent of depolarization $\rho$ is given by

$$
\rho=\frac{\Gamma_{\mathrm{SWM}}\left(\mathbf{e}^{\prime} \perp \mathbf{e}\right)}{\Gamma_{\mathrm{SWM}}\left(\mathbf{e}^{\prime} \| \mathbf{e}\right)}=\left|\frac{\left\{3 \alpha_{\lambda \lambda(\mu \mu v v)}-2 \alpha_{\lambda \mu(\lambda \mu v v)}\right\}}{\left\{\alpha_{\lambda \lambda(\mu \mu v v)}+4 \alpha_{\lambda \mu(\lambda \mu v v)}\right\}}\right|^{2}
$$

whose value must lie in the range $0 \leq \rho \leq 9$. If full permutational (Kleinman) index symmetry applies to the components of the nonlinear susceptibility tensor, Eq. (109) reduces to the result $\rho=\frac{1}{25}$. Departure of the degree of depolarization from this value thus registers invalidity of the Kleinman assumption.

Before further developing the theory to a form more directly suited to a different kind of experimental application, we outline why SWM is a mechanism allowed for all possible molecular symmetries. By inspection of the index symmetry in the radiation tensor, it is clear that a harmonic signal can derive only from that part of the sixth-rank polarizability $\boldsymbol{\alpha}_{\mathrm{SWM}}^{(6)}$ that is symmetric with respect to permutation among the four indices related to the absorbed pump 
photons, and also between the two indices relating to the two harmonic photons. Under the operations of the full rotation group $\mathrm{SO}(3)$, the group-theoretic representation of the tensor emerges as $2 D^{(0+)} \oplus D^{(1+)} \oplus 4 D^{(2+)} \oplus 2 D^{(3+)}+$ $3 D^{(4+)} \oplus D^{(5+)} \oplus D^{(6+)}$, accommodating a maximum of 90 independent components in the case of a molecule lacking any intrinsic symmetry. If full (Kleinman) index symmetry is assumed, the representation becomes $D^{(0+)} \oplus D^{(2+)} \oplus D^{(6+)}$, accounting for a total of just 28 independent components. In either case the crucial facet of the result is its incorporation of a $D^{(0+)}$ component, which invariably spans the totally symmetric representation of any point or space groups. Thus six-wave second-harmonic production entails a nonlinear polarizability that never vanishes for symmetry reasons; for example, it permits the process to be supported in centrosymmetric molecules or solids. Naturally, the six-wave process will be insignificant in media where the normal SHG process is allowed, since it derives from three orders higher perturbation theory.

Since the early 1990s, a number of studies on the generation of optically induced harmonics from isotropic suspensions of organic dyes have led to the characterization of SWM mechanisms (see, e.g., Refs. 88-90 and 92-95). However, it has generally been found experimentally expedient to induce the harmonic by seeding. This requires that samples be pumped not only with the four beams at the fundamental but also with a probe beam at the harmonic frequency, allowing for stimulated emission to enhance the interaction. The experimental setup is usually based on three distinct beams impinging on the sample. Of these, two are counterpropagating fundamental beams of frequency $\omega$, with the third at a frequency of $2 \omega$ stimulating the second harmonic into a specific mode satisfying the wavevector matching conditions. A schematic experimental geometry is illustrated in Fig. 13. Under such conditions the number of time-ordered diagrams is increased from the original 15 to $180(=6 ! /$ $2 ! 2 !)$, indicating a reduction in the extent of permutational symmetry among both the product radiation and molecular polarizability indices.

Referring to the experimental geometry of Fig. 13, we can assign the radiation modes $r_{1}=\left(\mathbf{k}_{1}, \lambda_{1}\right), r_{2}=\left(\mathbf{k}_{2} \lambda_{2}\right), r_{3}=\left(\mathbf{k}_{3}, \lambda_{3}\right)$, and $r_{4}=\left(\mathbf{k}_{4}, \lambda_{4}\right)$, recognizing that $\mathbf{k}_{1}=-\mathbf{k}_{2}$ and $\mathbf{k}_{3}=-\mathbf{k}_{4}$. Using the coherent representation for a SWM process, [Eq. (106)], and recognizing the appropriate index symmetry, we can write

$$
\begin{aligned}
\Gamma_{\mathrm{SWM}}= & \left(\frac{2 \pi}{\varepsilon_{0}^{6}}\right)\left(\frac{I_{1}}{2 c n_{\omega}}\right)^{2}\left(\frac{I_{2}}{2 c n_{\omega}}\right)^{2}\left(\frac{n_{\omega}+2}{3}\right)^{8} g_{1}^{(2)} g_{2}^{(2)}\left(\frac{I_{3}}{2 c n_{2 \omega}}\right)\left(\frac{n_{2 \omega}+2}{3}\right)^{2} \\
& \times \sum_{\mathbf{k}_{4}, \lambda_{4}}\left(\frac{v_{g}^{\prime} \omega_{k_{4}}}{2 c V n_{\omega_{4}}}\right)\left(\frac{n_{\omega_{4}}+2}{3}\right)^{2} \eta_{N} \delta\left(\hbar \omega_{k_{4}}+2 \hbar \omega-4 \hbar \omega\right) \\
& \times\left|\alpha_{i j(k l)(m n)}^{(6)}\left(-\omega_{k_{4}},-2 \omega ; \omega, \omega, \omega, \omega\right) \bar{e}_{i}^{(4)} \bar{e}_{j}^{(3)} e_{k}^{(2)} e_{l}^{(2)} e_{m}^{(1)} e_{n}^{(1)}\right|^{2}
\end{aligned}
$$



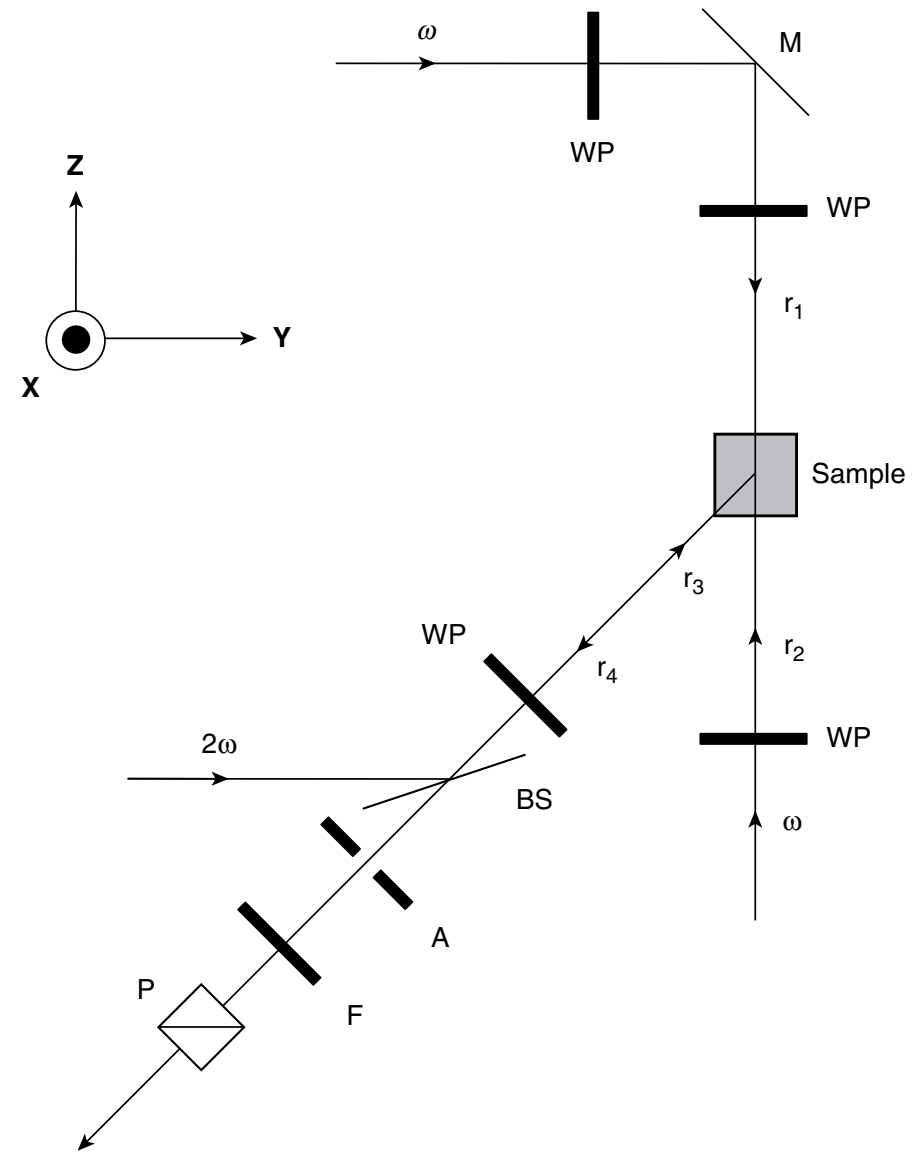

\section{Detector}

Figure 13. Schematic of a typical SWM experimental set-up. Counter-propagating fundamental beams of mode $r_{1}=\left(\mathbf{k}_{1}, \lambda_{1}\right)$ and $r_{2}=\left(\mathbf{k}_{2}, \lambda_{2}\right)$, each contribute two photons while the stimulating beam $r_{3}=\left(\mathbf{k}_{3}, \lambda_{3}\right.$ and signal beam $r_{4}=\left(\mathbf{k}_{4}, \lambda_{4}\right)$ each gain one photon. The laboratory axes are illustrated and other symbols represent: A-Aperture, BS-beam splitter, C-chopper, F-bandpass filter, P-polarizer, M-mirror and WP-wave plate.

Note the dependence of the rate on the intensity of the stimulating harmonic or seeding beam at $2 \omega$. On converting the sum over $\mathbf{k}_{4}$ to an integral, utilizing our knowledge of the delta function and converting to a harmonic intensity, we have

$$
\begin{aligned}
I_{\mathrm{SWM}}= & \frac{1}{\varepsilon_{0}^{6}(2 \pi)^{2}}\left(\frac{I_{1}}{2 c n_{\omega}}\right)^{2}\left(\frac{I_{2}}{2 c n_{\omega}}\right)^{2}\left(\frac{n_{\omega}+2}{3}\right)^{8} g_{1}^{(2)} g_{2}^{(2)}\left(\frac{I_{3}}{2 c}\right)\left(\frac{(2 \omega)^{4}}{2 c^{3}}\right)\left(\frac{n_{2 \omega}+2}{3}\right)^{4} \eta_{N} \\
& \times \sum_{\lambda_{4}}\left|\alpha_{i j(k l)(m n)}^{(6)}(-2 \omega,-2 \omega ; \omega, \omega, \omega, \omega) \bar{e}_{i}^{(4)} \bar{e}_{j}^{(3)} e_{k}^{(2)} e_{l}^{(2)} e_{m}^{(1)} e_{n}^{(1)}\right|^{2}
\end{aligned}
$$


where $g_{i}^{(2)}$ is the second-order degree of coherence of the $i$ th mode. Notice how the artificial dependence on the quantization volume disappears. The interesting features of the optical response are embedded in the molecular polarizability/ radiation tensor product. For an isotropic sample, we again have to perform a rotational average taken inside the modulus squared. Index symmetry exists only in the $k, l$ and $m, n$ index pairs, as indicated by parentheses. Therefore, on applying a sixth-rank average, we calculate the response as

$$
\left\langle I_{\mathrm{SWM}}\right\rangle=\frac{\kappa}{105} \sum_{i=6}^{6} E_{i} A_{i}
$$

Here $\kappa$ represents all the molecule-independent and polarization-independent constants gathered into a single parameter; the $E_{i}$ values represent the unique radiation polarization products

$$
\begin{array}{ll}
E_{1}=\left(\mathbf{e}^{(1)} \cdot \mathbf{e}^{(1)}\right)\left(\mathbf{e}^{(2)} \cdot \mathbf{e}^{(2)}\right)\left(\overline{\mathbf{e}}^{(3)} \cdot \overline{\mathbf{e}}^{(4)}\right) & E_{3}=\left(\mathbf{e}^{(1)} \cdot \mathbf{e}^{(2)}\right)\left(\mathbf{e}^{(1)} \cdot \overline{\mathbf{e}}^{(3)}\right)\left(\mathbf{e}^{(2)} \cdot \overline{\mathbf{e}}^{(4)}\right) \\
E_{2}=\left(\mathbf{e}^{(1)} \cdot \mathbf{e}^{(1)}\right)\left(\mathbf{e}^{(2)} \cdot \overline{\mathbf{e}}^{(3)}\right)\left(\mathbf{e}^{(2)} \cdot \overline{\mathbf{e}}^{(4)}\right) & E_{4}=\left(\mathbf{e}^{(1)} \cdot \mathbf{e}^{(2)}\right)\left(\mathbf{e}^{(1)} \cdot \overline{\mathbf{e}}^{(4)}\right)\left(\mathbf{e}^{(2)} \cdot \overline{\mathbf{e}}^{(3)}\right) \\
E_{3}=\left(\mathbf{e}^{(1)} \cdot \mathbf{e}^{(2)}\right)\left(\mathbf{e}^{(1)} \cdot \mathbf{e}^{(2)}\right)\left(\overline{\mathbf{e}}^{(3)} \cdot \overline{\mathbf{e}}^{(4)}\right) & E_{5}=\left(\mathbf{e}^{(1)} \cdot \overline{\mathbf{e}}^{(3)}\right)\left(\mathbf{e}^{(1)} \cdot \overline{\mathbf{e}}^{(4)}\right)\left(\mathbf{e}^{(2)} \cdot \mathbf{e}^{(2)}\right)
\end{array}
$$

and the $A_{i}$ values represent the molecular response, in the following format:

$$
\left[\begin{array}{l}
A_{1} \\
A_{2} \\
A_{3} \\
A_{4} \\
A_{5} \\
A_{6}
\end{array}\right]=\left[\begin{array}{rrrrrr}
8 & -5 & -5 & 4 & 4 & 5 \\
-5 & 11 & 4 & -6 & -6 & 4 \\
-5 & 4 & 11 & -6 & -6 & 4 \\
4 & -6 & -6 & 16 & 2 & -6 \\
4 & -6 & -6 & 1 & 16 & -6 \\
-5 & 4 & 4 & -6 & -6 & 11
\end{array}\right]\left[\begin{array}{l}
\boldsymbol{\alpha}_{1} \\
\boldsymbol{\alpha}_{2} \\
\boldsymbol{\alpha}_{3} \\
\boldsymbol{\alpha}_{4} \\
\boldsymbol{\alpha}_{5} \\
\boldsymbol{\alpha}_{6}
\end{array}\right]
$$

Here each independent molecular polarizability invariant is explicitly defined as

$$
\begin{array}{ll}
\boldsymbol{\alpha}_{1}=\alpha_{i i(j j)(k k)}^{(6)} & \boldsymbol{\alpha}_{4}=\boldsymbol{\alpha}_{i j(i k)(j k)}^{(6)} \\
\boldsymbol{\alpha}_{2}=\alpha_{i j(i j)(k k)}^{(6)} & \boldsymbol{\alpha}_{5}=\boldsymbol{\alpha}_{j i(i k)(j k)}^{(6)} \\
\boldsymbol{\alpha}_{3}=a_{i i(j k)(j k)}^{(6)} & \boldsymbol{\alpha}_{6}=a_{j k(i i)(j k)}^{(6)}
\end{array}
$$

In this format we can easily derive expressions for the signal intensity of the harmonic for arbitrary electric field polarizations. By assuming a laboratoryframe coordinate axis as illustrated in the experimental setup shown in Fig. 13, 


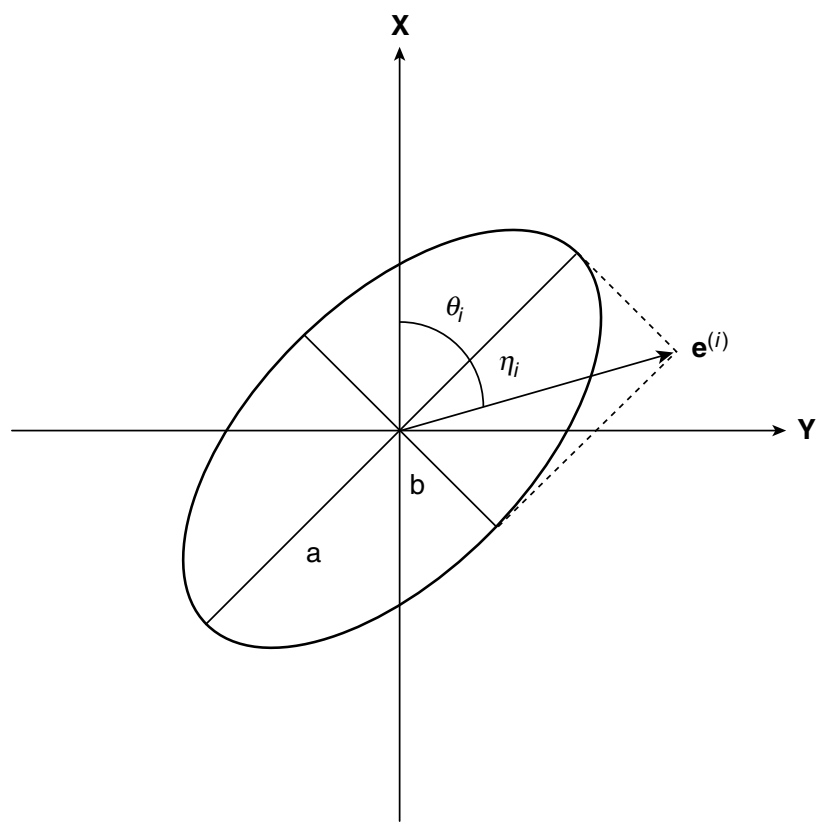

Figure 14. General representation for an arbitrarily polarized light beam, with polarization vector $\mathbf{e}^{(i)}$ as represented by Eq. (116). The definition of the azimuth $\theta_{i}$ and ellipticity $\eta_{i}=\tan ^{-1}(\mathrm{~b} /$ a) is as illustrated with respect to the $\mathbf{X}$ - and $\mathbf{Y}$-axis with the $\mathbf{Z}$-axis pointing into the plane of the paper.

the polarization vectors for the four fields, characterized by their azimuth and ellipticity as defined in Fig. 14, are concisely expressed as

$$
\begin{aligned}
\mathbf{e}^{(1)}= & \left(\cos \theta_{1} \cos \eta_{1}-i \sin \theta_{1} \sin \eta_{1}\right) \hat{\mathbf{x}}+\left(\sin \theta_{1} \cos \eta_{1}+i \cos \theta_{1} \sin \eta_{1}\right) \hat{\mathbf{y}} \\
\mathbf{e}^{(2)}= & \left(\cos \theta_{2} \cos \eta_{2}-i \sin \theta_{2} \sin \eta_{2}\right) \hat{\mathbf{x}}-\left(\sin \theta_{2} \cos \eta_{2}+i \cos \theta_{2} \sin \eta_{2}\right) \hat{\mathbf{y}} \\
\mathbf{e}^{(3)}= & \left(\cos \theta_{3} \cos \eta_{3}-i \sin \theta_{3} \sin \eta_{3}\right) \hat{\mathbf{x}}-\left(\sin \theta_{3} \cos \eta_{3}+i \cos \theta_{3} \sin \eta_{3}\right) \hat{\mathbf{y}} \\
& \times(\cos \delta \hat{\mathbf{y}}-\sin \delta \hat{\mathbf{z}}) \\
\mathbf{e}^{(4)}= & \left(\cos \theta_{4} \cos \eta_{4}-i \sin \theta_{4} \sin \eta_{4}\right) \hat{\mathbf{x}}+\left(\sin \theta_{4} \cos \eta_{4}+i \cos \theta_{4} \eta_{4}\right) \hat{\mathbf{y}} \\
& \times(\cos \delta \hat{\mathbf{y}}-\sin \delta \hat{\mathbf{z}})
\end{aligned}
$$

By placing a plane polarizer in the signal collection geometry $\left(\eta_{4}=0\right)$ and collecting the signal separately along $\hat{\mathbf{x}}\left(\theta_{4}=0\right)$ and $\hat{\mathbf{y}}\left(\theta_{4}=\pi / 2\right)$ directions, we are free to select any particular set of polarizations for the applied fields. As an example of the many controlled polarization plots possible, consider the applied 
fields all linearly polarized along the $\hat{\mathbf{x}}$ axis except for beam $\mathbf{e}^{(1)}$, which makes an angle $\theta_{1}$ to the others. The signal intensity then reduces from Eq. (112) to

$$
\begin{aligned}
\left\langle I_{\mathrm{SWM}}^{(\hat{\mathbf{x}})-\mathrm{pol}}\right\rangle & =\frac{\mathrm{K}}{105}\left|\left(A_{1}+A_{2}\right)+\left(A_{3}+A_{4}+A_{5}+A_{6}\right) \cos ^{2} \theta_{1}\right|^{2} \\
\left\langle I_{\mathrm{SWM}}^{(\hat{\mathbf{y}}-\mathrm{pol}}\right\rangle & =\frac{\mathrm{K}}{105}\left|\frac{1}{2}\left(A_{5}+A_{6}\right) \sin 2 \theta_{1} \cos \delta\right|^{2}
\end{aligned}
$$

where $\delta$ is the angle at which the seeding and signal fields propagate with respect to the laboratory $(\hat{\mathbf{x}}, \hat{\mathbf{y}})$ plane. The calculated signal for collection along the $\hat{\mathbf{x}}$ and $\hat{\mathbf{y}}$ axis can be plotted as a function of angle $\theta_{1}$. This is illustrated in Fig. 15. By performing a number of similarly designed experiments, detailed information on the six-wave mixing polarizability tensor can be extracted.

The seeding of molecular harmonics is in some sense a throwback to experiments where second harmonics were first observed in condensed-matter isotropic systems. For example, in glass fibers it was observed that a harmonic was produced after long exposures to fundamental frequency laser light $[99,100]$. It was later found that, by introduction of a low intensity seeding beam at the harmonic frequency, the onset of the harmonic in the glass was essentially instantaneous [101]. It was at this time that the proposal of a SWM mechanism

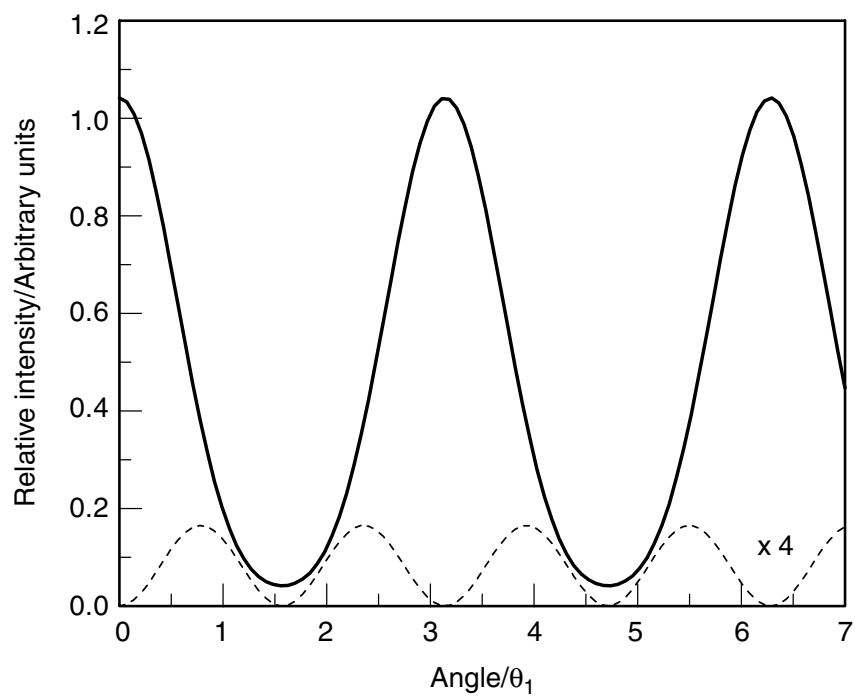

Figure 15. The solid and dotted lines represent theoretically calculated traces for SWM signals collected under the polarization conditions pertaining to Eqs. (117) and (118), respectively. The solid trace is normalized and the dotted line scaled by a factor of 4 . The results are calculated assuming all molecular parameters $A_{1}-A_{6}$ yield equal contributions. 
was first made [102,103]. However, it is necessary to accommodate additional features that are observed in the generation of harmonics from suspensions of organic dyes that absorb at the harmonic frequency. In such media the temporal behavior of the harmonic evolution is generally considered consistent with some degree of molecular alignment as a result of the optical perturbations to the environment [104]. Such perturbations would necessarily be physically distinct from the process of harmonic production, although engendered by the same optical input beams. This represents a move away from the instantaneous SWM mechanism, allowed for all molecular symmetries, focusing instead on timedependent molecular orientational symmetry breaking. Here we illustrate how, with a resonant seeding harmonic, the concept of a molecular population diffraction grating can be employed as an alternative and more readily comprehensible means of eliciting the physics of harmonic evolution. This is a quantum optical effect that does not require the invocation of light-induced orientation. Its foundation is based on a selective absorption process that is a direct result of a molecular ensemble initially having an isotropic array of molecules.

The first task then is to show that, in the presence of the two writing beams $r_{2}$ and $r_{3}$, the created population grating is of just the correct periodicity to efficiently generate phase-matched second-harmonic photons from the probe beam $r_{1}$. As a result, the $r_{4}$ signal photons emerge at the second-harmonic frequency and propagate in the direction exactly opposite that of seeding beam $r_{3}$, according to the dictates of wavevector matching. We shall suppose that the seeding pulses from modes $r_{2}$ and $r_{3}$ are coincident with the sample at time $t=0$, and then at $t=\tau$ the pulse from the probe beam $r_{1}$ arrives. The sample is absorbing at the harmonic frequency, and so transition to the excited state is expected. Nonetheless, there are two ways to accomplish this in the presence of the two differing input frequencies: (1) two-photon absorption of photons solely from the fundamental beam and (2) single-photon absorption of photons from the harmonic beam. (This principle was first considered in connection with ionization processes by Baranova and Zel'dovich, [105].) We thus need to consider two time orderings as shown in Fig. 16. The matrix element (quantum probability amplitude) for the transition in a particular molecule $\xi$ is thus written as

$$
M_{f i}^{(\xi)}=M_{f i}^{(\xi, a)}+M_{f i}^{(\xi, b)}
$$

where $M_{f i}^{(\xi, a)}$ is the matrix element for graph (a) of Fig. 16 and $M_{f i}^{(\xi, b)}$ is that for graph (b). By rigorously following the procedures for forming the matrix elements as outlined in earlier sections, these quantities can be written as

$$
M_{f i}^{(\xi, a)}=\left(\frac{\hbar v_{g} \omega_{k}}{2 c \varepsilon_{0} V n_{\omega_{k}}}\right)\left(\frac{n_{\omega_{k}}^{2}+2}{3}\right)^{2} \sqrt{\left\langle q_{2}\right\rangle\left\langle\left(q_{2}-1\right)\right\rangle} \alpha_{(i j)}^{10}(\omega, \omega) e_{i}^{(2)} e_{j}^{(2)} e^{i 2 \mathbf{k}_{2} \cdot \mathbf{R}_{\xi}}
$$




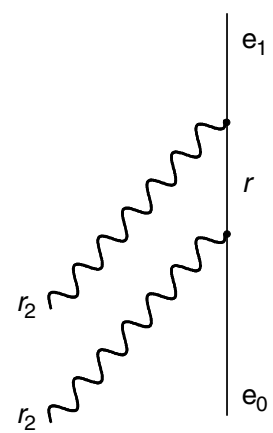

(a)

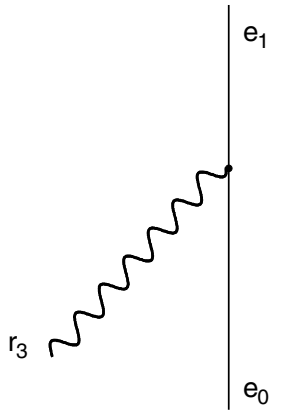

(b)

Figure 16. The time-ordered diagrams associated with the formation of an appropriate molecular grating for SHG. The two writing beams $r_{2}$ and $r_{3}$ populate the upper electronic state via two- and single-photon absorption respectively.

and

$$
M_{f i}^{(\xi, b)}=i\left(\frac{\hbar v_{g}^{\prime} \omega_{k^{\prime}}^{\prime} k^{\prime}}{2 c V \varepsilon_{0} n_{\omega_{k^{\prime}}}}\right)^{1 / 2}\left(\frac{n_{\omega_{k^{\prime}}^{\prime}}^{2}+2}{3}\right) \sqrt{\left\langle q_{3}\right\rangle} \mu_{i}^{10} e_{i}^{(3)} e^{i \mathbf{k}_{3} \cdot \mathbf{R}_{\xi}}
$$

In these equations the position of the molecule is described by the vector $\mathbf{R}_{\xi}$; the wavevectors of the two beams of modes $r_{2}$ and $r_{3}$ are $\mathbf{k}_{2}$ and $\mathbf{k}_{3}$ respectively, with $\left\langle q_{2}\right\rangle$ and $\left\langle q_{3}\right\rangle$ the corresponding mean photon numbers (mode occupancies); and $\mathbf{e}^{(n)}$ is a unit vector describing the polarization state of mode $r_{n}$. In deriving Eqs. (120) and (121), the state vectors describing the radiation fields have been assumed to be coherent laser states, and so, for example, $\left\langle q_{2}\right\rangle=\left\langle\alpha^{(2)}|\hat{n}| \alpha^{(2)}\right\rangle$, where $\left|\alpha^{(2)}\right\rangle$ is the coherent state representing mode 2 and $\hat{n}$ is the number operator; a similar expression may be written for $\left\langle q_{3}\right\rangle$. Also, the molecular parameters apparent in Eqs. (120) and (121) are the components of the transition dipole, $\mu_{i}^{10}$, and the index-symmetric second-order molecular transition tensor, $\alpha_{(i j)}^{10}(\omega, \omega)$.

The rate at which the excited state is populated is once again given by recourse to the Golden Rule [Eq. (51)] and clearly three contributions are apparent:

$$
\Gamma=\frac{2 \pi}{\hbar}\left|M_{f i}^{(\xi)}\right|^{2} \rho_{F}^{(1)}=\Gamma_{1}+\Gamma_{2}+\Gamma_{3}
$$


where

$$
\begin{aligned}
\Gamma_{1}= & \frac{2 \pi \rho_{F}^{(1)}}{\hbar}\left(\frac{\hbar v_{g} \omega_{k}}{2 c \varepsilon_{0} V n_{\omega_{k}}}\right)^{2}\left(\frac{n_{\omega_{k}}^{2}+2}{3}\right)^{4}\left\langle q_{2}\right\rangle\left\langle\left(q_{2}-1\right)\right\rangle\left|\alpha_{(i j)}^{(10)} e_{i}^{(2)} e_{j}^{(2)}\right|^{2} \\
\Gamma_{2}= & \frac{2 \pi \rho_{F}^{(1)}}{\hbar}\left\{-i\left(\frac{\hbar v_{g} \omega_{k}}{2 c \varepsilon_{0} V n_{\omega_{k}}}\right)\left(\frac{n_{\omega_{k}}^{2}+2}{3}\right)^{2}\left(\frac{\hbar v_{g}^{\prime} \omega_{k^{\prime}}^{\prime}}{2 c \varepsilon_{0} V n_{\omega_{k^{\prime}}^{\prime}}}\right)^{1 / 2}\left(\frac{n_{\omega_{k^{\prime}}^{\prime}}^{2}+2}{3}\right)\right. \\
& \left.\sqrt{\left\langle q_{3}\right\rangle\left\langle q_{2}\right\rangle\left\langle\left(q_{2}-1\right)\right\rangle} \alpha_{(i j)}^{10} \bar{\mu}_{k}^{10} e_{i}^{(2)} e_{j}^{(2)} \bar{e}_{k}^{(3)} e^{i\left(2 \mathbf{k}_{2}-\mathbf{k}_{3}\right) \cdot \mathbf{R}_{\xi}}+\mathrm{c.c}\right\}
\end{aligned}
$$

and

$$
\Gamma_{3}=\frac{2 \pi \rho_{F}^{(1)}}{\hbar}\left(\frac{\hbar v_{g}^{\prime} \omega_{k^{\prime}}^{\prime}}{2 c \varepsilon_{0} V n_{\omega_{k^{\prime}}^{\prime}}}\right)\left(\frac{n_{\omega_{k}}^{2}+2}{3}\right)^{2}\left\langle q_{3}\right\rangle\left|\mu_{i}^{10} e_{i}^{(3)}\right|^{2}
$$

As the transition is to a particular molecular electronic manifold, we have utilized the convenient density of states representation in the expressions. We see that the rate at which the exited state is populated depends on the position of the molecule, through $\Gamma_{2}$ - and also on the molecular orientation, through the matrix elements. It is this $\Gamma_{2}$ term that produces the grating within the sample. We note here that the periodicity of the grating, determined by $e^{i\left(2 \mathbf{k}_{2}-\mathbf{k}_{3}\right) \cdot \mathbf{R}_{\xi}} \equiv e^{-i\left(2 \mathbf{k}_{1}+\mathbf{k}_{3}\right) \cdot \mathbf{R}_{\xi}}$, is exactly that required for phase-matched second-harmonic generation from the probe beam, where the signal is created in precisely the opposite direction to the harmonic pump beam.

Thus far we have a position-dependent rate $\Gamma\left(\mathbf{R}_{\xi}\right)$ at which the upper state is populated during application of the two writing beams. If we take the effective time for which the beams are applied as $\Delta t$, the probability that molecule $\xi$ is excited immediately after the pulses have passed is $P\left(\mathbf{R}_{\xi}\right)=\Gamma\left(\mathbf{R}_{\xi}\right) \Delta t$. The probe pulse arrives after a delay of $\tau(>\Delta t)$ seconds, during which time the molecule, if excited, may relax. We suppose that it relaxes to the ground state via a simple exponential decay. At time $\tau$ the probability that the molecule is excited is hence

$$
P\left(\mathbf{R}_{\xi}, \tau\right)=\Gamma\left(\mathbf{R}_{\xi}\right) \Delta t e^{-k_{10}(\tau-\Delta t)}
$$

where $k_{10}$ is the decay constant. It is useful to assume that the molecules remain clamped in between light pulses, so that we can ignore any movement (rotational or translational) that may occur during these finite periods. This physically reasonable assumption is primarily made for calculational expediency; it may be 


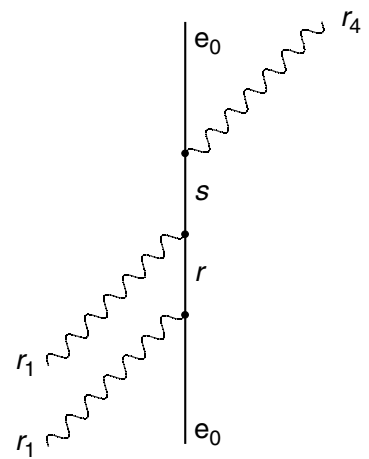

(a)

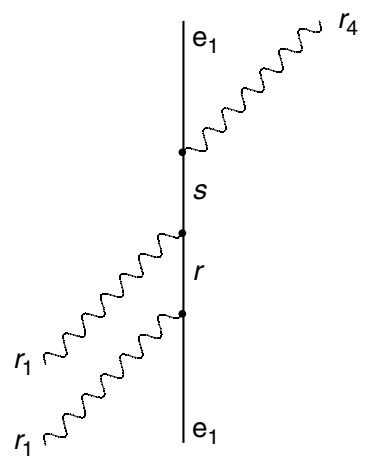

(b)

Figure 17. Representative time-ordered diagrams illustrating the harmonic formation from molecules in (a) the ground electronic state $\mathrm{e}_{0}$ and (b) the excited state $\mathrm{e}_{1}$.

dispensed with, but only at the cost of substantially increased complexity [93]. This means that the probe pulse encounters the associated population distribution in the sample and the harmonic is then produced from it. Again two possibilities arise, as illustrated in Fig. 17 (in which only the dominant of three contributory time orderings is shown). Writing as $M_{f i}^{\prime(\xi, a)}$ and $M_{f i}^{\prime(\xi, b)}$ the quantum-optical matrix elements for these component processes, the effective matrix element for harmonic production will be

$$
M_{f i}^{\prime(\xi)}=\left(1-P\left(\mathbf{R}_{\xi}, \tau\right)\right) M_{f i}^{\prime(\xi, a)}+P\left(\mathbf{R}_{\xi}, \tau\right) M_{f i}^{\prime(\xi, b)}
$$

reflecting a statistical weighting of the appropriate quantum amplitudes. For a two-level system, Eq. (127) is exact, and follows from use of the completeness relation for the molecular states. The two components involved in the harmonic generation process are, in fact, identical in terms of the photonics, differing only in their molecular mediation, and so we have

$$
\begin{aligned}
M_{f i}^{\prime}(\xi, a)= & -i\left(\frac{\hbar v_{g} \omega_{k}}{2 c \varepsilon_{0} V n_{\omega_{k}}}\right)\left(\frac{n_{\omega_{k}}^{2}+2}{3}\right)^{2}\left(\frac{\hbar v_{g}^{\prime} \omega_{k^{\prime}}^{\prime}}{2 c \varepsilon_{0} V n_{\omega_{k^{\prime}}^{\prime}}}\right)^{1 / 2}\left(\frac{n_{\omega_{k^{\prime}}^{\prime}}^{2}+2}{3}\right) \\
& \times \sqrt{\left\langle q_{1}\right\rangle\left(\left\langle q_{1}\right\rangle-1\right)} \beta_{i(j k)}^{00} \bar{e}_{i}^{(4)} e_{j}^{(1)} e_{k}^{(1)} e^{i\left(-2 \mathbf{k}_{2}+\mathbf{k}_{3}\right) \cdot \mathbf{R}_{\xi}}
\end{aligned}
$$

and

$$
\begin{aligned}
M_{f i}^{\prime(\xi, b)}= & -i\left(\frac{\hbar v_{g} \omega_{k}}{2 c \varepsilon_{0} V n_{\omega_{k}}}\right)\left(\frac{n_{\omega_{k}}^{2}+2}{3}\right)^{2}\left(\frac{\hbar v_{g}^{\prime} \omega_{k^{\prime}}^{\prime}}{2 c \varepsilon_{0} V n_{\omega_{k^{\prime}}}}\right)^{1 / 2}\left(\frac{n_{\omega_{k^{\prime}}^{\prime}}^{2}+2}{3}\right) \\
& \times \sqrt{\left\langle q_{1}\right\rangle\left(\left\langle q_{1}\right\rangle-1\right)} \beta_{i(j k)}^{11} \bar{e}_{i}^{(4)} e_{j}^{(1)} e_{k}^{(1)} e^{i\left(-2 \mathbf{k}_{2}+\mathbf{k}_{3}\right) \cdot \mathbf{R}_{\xi}}
\end{aligned}
$$


where $\left\langle q_{1}\right\rangle$ is the mean number of photons in mode $r_{1}$. The index-symmetric hyperpolarizbilities are exactly as those given in previous sections (where the upper level was designated $u$ ). They are distinguished by the fact that the harmonic stems from the labeled states 0 and 1, respectively; as before, the repeated superscripts indicate that the molecules return to their initial state following the interaction. As shown in the last section, a two-level model would require that the hyperpolarizability of the upper level be precisely minus that of the ground state, as in Eq. (96). In the SWM systems of experimental interest, the two-level model is too restrictive; however, it can certainly be anticipated that the upper- and lower-level hyperpolarizabilities will substantially differ, a feature that proves crucial for the following analysis.

To continue, we now compile the total matrix element for SHG from the ensemble through

$$
\begin{aligned}
M_{f i}^{\prime}= & -i\left(\frac{\hbar v_{g} \omega_{k}}{2 c \varepsilon_{0} V n_{\omega_{k}}}\right)\left(\frac{n_{\omega_{k}}^{2}+2}{3}\right)^{2}\left(\frac{\hbar v_{g}^{\prime} \omega_{k^{\prime}}^{\prime}}{2 c \varepsilon_{0} V n_{\omega_{k^{\prime}}^{\prime}}}\right)^{1 / 2}\left(\frac{n_{\omega_{k^{\prime}}^{\prime}}^{2}+2}{3}\right) \\
& \times \sqrt{\left\langle q_{1}\right\rangle\left(\left\langle q_{1}\right\rangle-1\right)} \bar{e}_{i}^{(4)} e_{j}^{(1)} e_{k}^{(1)} \sum_{\xi}\left\{\beta_{i(j k)}^{00}+P\left(\mathbf{R}_{\xi}, \tau\right) \Delta \beta_{i(j k)}\right\} e^{i\left(-2 \mathbf{k}_{2}+\mathbf{k}_{3}\right) \cdot \mathbf{R}_{\xi}}
\end{aligned}
$$

where the difference in the hyperpolarizabilities between the upper and lower states has been written

$$
\Delta \beta_{i(j k)}=\beta_{i(j k)}^{11}-\beta_{i(j k)}^{00}
$$

The rate of production of SHG from the ensemble given by the Golden Rule is

$$
R=\frac{2 \pi \rho_{F}^{(2)}}{\hbar}\left|\sum_{\xi} M_{f i}^{\prime(\xi)}\right|^{2}
$$

where $\rho_{F}^{(2)}$ is the density of states for the second (reading) process. Taking an orientational average and effecting the usual split into incoherent (single site) and coherent (multi-site interference) terms, we have

$$
\langle R\rangle=\frac{2 \pi \rho_{F}^{(2)}}{\hbar}\left\langle\sum_{\xi}\left|M_{f i}^{\prime(\xi)}\right|^{2}+\sum_{\xi \neq \xi^{\prime}} M_{f i}^{\prime(\xi)} \bar{M}_{f i}^{\prime\left(\xi^{\prime}\right)}\right\rangle
$$

The dominant contribution to SHG is hence the coherent term

$$
R_{\mathrm{coh}}=\frac{2 \pi \rho_{F}^{(2)}}{\hbar} \sum_{\xi \neq \xi^{\prime}}\left\langle M_{f i}^{\left(\xi^{\prime}\right)}\right\rangle\left\langle\bar{M}_{f i}^{\prime\left(\xi^{\prime}\right)}\right\rangle
$$

where we have assumed that differing molecules in the solution are orientationally uncorrelated, as is the case for the majority of pairs in the 
system. For any one particular molecule, one can simply employ Eq. (130), excluding the sum over $\xi$. Referring back to that equation and effecting the orientational average for individual molecules leads to disappearance of the first term within the parenthesis, as is usual for SHG in isotropic media. The second term, however, contains "hidden" orientational factors through $P\left(\mathbf{R}_{\xi}, \tau\right)$, as a result of which the average is nonzero. Using Eq. (126) we thus have

$$
\left\langle\Gamma\left(\mathbf{R}_{\xi}\right) \Delta t e^{-k_{10}(\tau-\Delta t)} \Delta \beta_{i(j k)}\right\rangle=\left\langle\left(\Gamma_{1}+\Gamma_{2}+\Gamma_{3}\right) \Delta \beta_{i(j k)}\right\rangle \Delta t e^{-k_{10}(\tau-\Delta t)}
$$

Of the three contributory terms, it is $\Gamma_{2}$ that is responsible for the observed signal, as it is the only term to exhibit the necessary phase matching when inserted into Eq. (130). We thus ignore the other two terms in (133). The assumption is justified by experiments where no SHG signal is observed if either of the writing beams $r_{2}$ or $r_{3}$ is blocked [106]. Using the $\Gamma_{2}$ term in Eq. (133), we now find

$$
\begin{aligned}
\left\langle\Gamma\left(\mathbf{R}_{\xi}\right) \Delta t e^{-k_{10}(\tau-\Delta t)} \Delta \beta_{i(j k)}\right\rangle= & i \frac{2 \pi \rho_{F}^{(1)}}{\hbar}\left(\frac{\hbar v_{g} \omega_{k}}{2 c \varepsilon_{0} V n_{\omega_{k}}}\right)\left(\frac{n_{\omega_{k}}^{2}+2}{3}\right)^{2}\left(\frac{\hbar v_{g}^{\prime} \omega_{k^{\prime}}^{\prime}}{2 c \varepsilon_{0} V n_{\omega_{k^{\prime}}^{\prime}}}\right)^{1 / 2} \\
& \times\left(\frac{n_{\omega_{k^{\prime}}^{\prime}}^{2}+2}{3}\right)\left[\left\langle q_{3}\right\rangle\left\langle q_{2}\right\rangle\left(\left\langle q_{2}\right\rangle-1\right)\right]^{1 / 2} \\
& \times\left\langle\left\{\alpha_{(l m)}^{10} \bar{\mu}_{n}^{10} e_{l}^{(2)} e_{m}^{(2)} \bar{e}_{n}^{(3)} e^{i\left(2 \mathbf{k}_{2}-\mathbf{k}_{3}\right) \cdot \mathbf{R}_{\xi}}+\text { c.c. }\right\}\right. \\
& \left.\times \Delta \beta_{i(j k)}\right\rangle \Delta t e^{-k_{10}(\tau-\Delta t)}
\end{aligned}
$$

with the exponential explicitly exhibiting the phase-matching (and the complex conjugate term accounting for the fact that SHG can be produced from a fundamental beam propagating in the opposite direction, as also observed experimentally). The phase-matched, orientationally averaged matrix element is hence

$$
\begin{aligned}
\left\langle M_{f i}^{\prime(\xi)}\right\rangle= & \frac{\pi \rho_{F}^{(1)}}{\hbar}\left(\frac{\hbar v_{g} \omega_{k}}{2 c \varepsilon_{0} V n_{\omega_{k}}}\right)^{2}\left(\frac{n_{\omega_{k}}^{2}+2}{3}\right)^{4}\left(\frac{\hbar v_{g}^{\prime} \omega_{k^{\prime}}^{\prime}}{2 c \varepsilon_{0} V n_{\omega_{k^{\prime}}^{\prime}}}\right)\left(\frac{n_{\omega_{k^{\prime}}^{\prime}}^{2}+2}{3}\right)^{2} \\
& \times \sqrt{\left\langle q_{3}\right\rangle\left\langle q_{2}\right\rangle\left(\left\langle q_{2}\right\rangle-1\right)\left\langle q_{1}\right\rangle\left(\left\langle q_{1}\right\rangle-1\right)} \\
& \times\left\langle\alpha_{(l m)}^{10} \bar{\mu}_{n}^{10} \Delta \beta_{i(j k)}\right\rangle e_{l}^{(2)} e_{m}^{(2)} \bar{e}_{n}^{(3)} \bar{e}_{i}^{(4)} e_{j}^{(1)} e_{k}^{(1)} \Delta t e^{-k_{10}(\tau-\Delta t)}
\end{aligned}
$$

which is necessarily position-independent, so that the phase-matching double sum in Eq. (132) can be evaluated for the ensemble of $N$ molecules as $N(N-1) \approx N^{2}$ for large $N$. The resulting rate of SHG production is

$$
\begin{aligned}
R_{\mathrm{coh}}= & \frac{2 \pi^{3}\left(\rho_{F}^{(1)}\right)^{2} \rho_{F}^{(2)}}{\hbar^{3}}(N \Delta t)^{2}\left(\frac{\hbar v_{g} \omega_{k}}{2 c \varepsilon_{0} V n_{\omega_{k}}}\right)^{4}\left(\frac{n_{\omega_{k}}^{2}+2}{3}\right)^{8}\left(\frac{\hbar v_{g}^{\prime} \omega_{k^{\prime}}^{\prime}}{2 c \varepsilon_{0} V n_{\omega_{k^{\prime}}^{\prime}}}\right)^{2}\left(\frac{n_{\omega_{k^{\prime}}}^{2}+2}{3}\right)^{4} \\
& \times\left\langle q_{3}\right\rangle\left\langle q_{2}\right\rangle\left(\left\langle q_{2}\right\rangle-1\right)\left\langle q_{1}\right\rangle\left(\left\langle q_{1}\right\rangle-1\right) \\
& \times\left|\left\langle\alpha_{(l m)}^{10} \bar{\mu}_{n}^{10} \Delta \beta_{i(j k)}\right\rangle e_{l}^{(2)} e_{m}^{(2)} \bar{e}_{n}^{(3)} \bar{e}_{i}^{(4)} e_{j}^{(1)} e_{k}^{(1)}\right|^{2} e^{-2 k_{10}(\tau-\Delta t)}
\end{aligned}
$$


Casting the result in terms of the mean intensities of the beams, with $\omega_{k^{\prime}}^{\prime} \equiv 2 \omega_{k}$, the final expression for coherent SHG from the grating may be written as

$$
\begin{aligned}
I_{\mathrm{sig}}^{(2 \omega)}= & \frac{\left(\rho_{F}^{(1)}\right)^{2}\left(k^{\prime}\right) g_{1}^{(2)} g_{2}^{(2)}(N \Delta t)^{2}}{256 \hbar^{2} c^{5} \varepsilon_{0}^{6}}\left(I_{1}^{(\omega)} I_{2}^{(\omega)}\right)^{2} I_{3}^{(2 \omega)} \\
& \times\left|\left\langle\alpha_{(l m)}^{10} \bar{\mu}_{n}^{10} \Delta \beta_{i(j k)}\right\rangle e_{l}^{(2)} e_{m}^{(2)} \bar{e}_{n}^{(3)} \bar{e}_{i}^{(4)} e_{j}^{(1)} e_{k}^{(1)}\right|^{2} e^{-2 k_{10}(\tau-\Delta t)}
\end{aligned}
$$

where $I_{n}^{(\omega)}$ is the mean intensity of the $n$th beam of frequency $\omega$ and $g_{n}^{(2)}$ is its degree of second-order coherence. Equation (136) thus exhibits the correct dependence on the intensities of the three input beams (quadratic with respect to the two fundamental beams and linear in the harmonic writing beam) and also the sample density $\left(I_{\text {sig }}^{(2 \omega)} \propto N^{2}\right)$. Dynamically this equation yields a simple exponential decay due to relaxation of the molecules from the excited to ground state; the timescale for the decay is therefore governed by the intrinsic fluorescence lifetime.

The polarization dependence of Eq. (136) is exactly that found previously for the case of coincident pulses, represented herein as Eq. (111). It is therefore interesting to compare the two results and their respective dependences on the molecular tensor components. Evaluating the sixth-rank average for Eq. (136), we find

$$
\left\langle I_{\mathrm{sig}}^{(2 \omega)}\right\rangle=\frac{\left(\rho_{F}^{(1)}\right)^{2}\left(k^{\prime}\right)^{3} g_{1}^{(2)} g_{2}^{(2)}(N \Delta t)^{2}}{256 \hbar^{2} c^{5} \varepsilon_{0}^{6}}\left(I_{1}^{(\omega)} I_{2}^{(\omega)}\right)^{2} I_{3}^{(2 \omega)}\left|\frac{1}{105} \sum_{i=1}^{6} E_{i} A_{1}^{\prime}\right|^{2} e^{-2 k_{10}(\tau-\Delta t)}
$$

where the modified linear matrix $\mathbf{A}^{\prime}$ now contains a molecular response through

$$
\left[\begin{array}{l}
A_{1}^{\prime} \\
A_{2}^{\prime} \\
A_{3}^{\prime} \\
A_{4}^{\prime} \\
A_{5}^{\prime} \\
A_{6}^{\prime}
\end{array}\right]=\left[\begin{array}{rrrrrr}
8 & -5 & -5 & 4 & 4 & 5 \\
-5 & 11 & 4 & -6 & -6 & 4 \\
-5 & 4 & 11 & -6 & -6 & 4 \\
4 & -6 & -6 & 16 & 2 & -6 \\
4 & -6 & -6 & 2 & 16 & -6 \\
-5 & 4 & 4 & -6 & -6 & 11
\end{array}\right]\left[\begin{array}{l}
\bar{\mu}_{\gamma}^{10} \alpha_{(\beta \beta)}^{10} \Delta \beta_{\gamma(\alpha \alpha)} \\
\bar{\mu}_{\beta}^{10} \alpha_{(\beta \gamma)}^{10} \Delta \beta_{\gamma(\alpha \alpha)} \\
\bar{\mu}_{\gamma}^{10} \alpha_{(\alpha \beta)}^{10} \Delta \beta_{\gamma(\alpha \beta)} \\
\bar{\mu}_{\beta}^{10} \alpha_{(\alpha \gamma)}^{10} \Delta \beta_{\gamma(\alpha \beta)} \\
\bar{\mu}_{\gamma}^{10} \alpha_{(\alpha \gamma)}^{10} \Delta \beta_{\beta(\alpha \beta)} \\
\bar{\mu}_{\alpha}^{10} \alpha_{(\gamma \gamma)}^{10} \Delta \beta_{\beta(\alpha \beta)}
\end{array}\right]
$$

The linear matrix $\mathbf{E}$ is exactly that as previously defined, in Eq. (113). The polarization characteristics are similar in form to those of the coincident-pulse case. Hence, although one would not expect them to be exactly the same because 
of their different dependence on molecular properties, polarization analysis is unlikely to unambiguously differentiate the contributory mechanisms. The latest analysis shows experimental results exactly in agreement with this theory [93].

\section{CONCLUSION}

In this review we have described some of the advances in the quantum electrodynamical formulation of theory for molecular photonics. We have shown how the framework described in an earlier review has now been extended to new areas of application, and reformulated for application to real dispersive media-as reflected in the new treatment of refractive, dissipative, and resonance properties. With all its conceptual splendor, conventional quantum optics has not generally been pursued at this level of detail on its dielectric host, and it is our hope that this work will help match its precepts with quantitative accuracy. Applications of the new theory have revealed new quantum optical features in two quite different aspects of the familiar process of second harmonic generation, one operating through local coherence within small particles and the other, a coherence between the quantum amplitudes for fundamental and harmonic excitation. Where the salient experiments have been performed, they exactly match the theoretical predictions. The theoretical foundation we have discussed therefore shows promise for the delivery of accurate insights into other optical processes yet to be characterized, and it should be well placed to facilitate the determination of meaningful data from the associated experiments.

\section{Acknowledgments}

We are pleased to acknowledge helpful comments and insights from friends and colleagues at various stages of preparing this review, and especially from Robert Jenkins, Gediminas Juzeliunas, Geoff Stedman, and T. Thirunamachandran.

\section{References}

1. D. L. Andrews, Modern Nonlinear Optics, in M. Evans and S. Kielich (Eds.), Adv. Chem. Phys. 85 (Part 2), 545-606.

2. T. Kinoshita, Quantum Electrodynamics, World Scientific, River Edge, NJ 1990.

3. E. A. Power, Introductory Quantum Electrodynamics, Longmans, London, 1964.

4. S. Haroche, in J. Dalibard, J.-M. Raimond and J. Zinn-Justin (Eds.), Fundamental Systems in Quantum Optics, Elsevier, Amsterdam, 1992.

5. P. W. Milonni, The Quantum Vacuum, Academic, San Diego, 1994.

6. G. Compagno, R. Passante, and F. Persico, Atom-Field Interactions and Dressed Atoms, Cambridge Univ. Press, 1995.

7. S. M. Barnett, A. Aspect, and P. W. Milonni, J. Phys. B, Atom. Mol. Opt. Phys. 33, L143-L149 (2000).

8. V. Chernyak and S. Mukamel, J. Chem. Phys. 100, 2953-2974 (1994).

9. D. L. Andrews and P. Allcock, Chem. Soc. Rev. 24, 259-265 (1995). 
10. G. Juzeliunas and D. L. Andrews, Adv. Chem. Phys. 112, 357-410 (2000).

11. G. E. Stedman, private communication, 2000.

12. P. W. Milonni, in S. Diner, D. Fargue, G. Lochak, and F. Selleri (Eds.), The Wave-Particle Dualism, Reidel, Dordrecht, 1984.

13. R. G. Woolley, Int. J. Quantum Chem. 74, 531-545 (1999).

14. R. G. Woolley, (a) Proc. Roy. Soc. Lond. A456, 1803-1819; 2000; (b) R. G. Woolley, private communication, 2000.

15. D. P. Craig and T. Thirunamachandran, Molecular Quantum Electrodynamics Academic, London, 1984.

16. B. J. Dalton, E. S. Guerra, and P. L. Knight, Phys. Rev. A 54, 2292-2313 (1996).

17. M. Göppert-Mayer, Ann. Physik 9, 273-294 (1931).

18. E. A. Power and S. Zienau, Phil. Trans. Roy. Soc. Lond. Ser. A 251, 427-454 (1959).

19. R. G. Woolley, Proc. Roy. Soc. Lond. A321, 557-572 (1971).

20. M. Babiker, E. A. Power, and T. Thirunamachandran, Proc. Roy. Soc. Lond. A338, 235-249 (1974).

21. E. A. Power and T. Thirunamachandran, Am. J. Phys. 46, 370-378 (1980).

22. E. A. Power and T. Thirunamachandran, Phys. Rev. A 60, 4936-4942 (1990).

23. B. J. McKenzie and G. E. Stedman, J. Phys. C, Solid State Phys. 12, 5062-5075 (1970).

24. S. Naguleswaran, Ph.D. thesis, Univ. Canterbury, NZ, 1998.

25. G. Juzeliunas and D. L. Andrews, Phys. Rev. B 49, 8751-8763 (1994).

26. P. W. Milonni, J. Mod. Opt. 42, 1991-2004 (1995).

27. G. Juzeliunas, Phys. Rev. A 53, 3543-3558 (1996).

28. C. Cohen-Tannoudji, J. Dupont-Roc, and G. Grynberg, Atom-Photon Interactions, Wiley, London, 1992.

29. S. Mukamel, Principles of Nonlinear Optical Spectroscopy, Oxford Univ. Press, Oxford, 1995.

30. L. C. Davila-Romero, Ph.D. thesis, Univ. East Anglia, 1999.

31. P. O. Löwdin, in C. H. Wilcox (Ed.), Perturbation Theory and Its Applications in Quantum Mechanics, Wiley, New York, 1965.

32. E. Fermi, Nuclear Physics, Univ. Chicago Press, Chicago, 1950.

33. P. A. M. Dirac, The Principles of Quantum Mechanics, Clarendon Press, Oxford, 1958.

34. L. I. Schiff, Quantum Mechanics, 3rd ed., McGraw-Hill, New York, 1968.

35. R. D. Jenkins and D. L. Andrews, J. Phys. Chem. A 102, 10834-10842 (1998).

36. W. H. Louisell, Quantum Statistical Properties of Radiation, Wiley, New York, 1973, pp. 104109.

37. R. Loudon, The Quantum Theory of Light, 2nd ed., Clarendon, Oxford, 1983.

38. D. Marcuse, Principles of Quantum Electronics, Academic, New York, 1980.

39. D. L. Andrews, J. Phys. B, Atom. Mol. Phys. 11, 2655-2664 (1978).

40. J. M. Manley and H. E. Rowe, Proc. Inst. Radio Eng. 47, 2115-2116 (1959).

41. H. Haken, Laser Theory, Springer, Berlin, 1984, p. 91.

42. D. M. Bishop and D. W. DeKee, J. Chem. Phys. 104, 9876-9887 (1996).

43. G. Wagnière, Appl. Phys. B 41, 169-172 (1986).

44. D. S. Elliott and J. F. Ward, Mol. Phys. 51, 45-63 (1984).

45. D. M. Bishop, Rev. Mod. Phys. 62, 343-374 (1990). 
46. D. M. Bishop and B. Kirtman, J. Chem. Phys. 95, 2646-2658 (1991).

47. Q. L. Zhou, J. R. Heflin, K. Y. Wong, O. Zamani-Khamiri, and A. F. Garito, Phys. Rev. A 43, 1673-1676 (1991).

48. J. R. Heflin, D. C. Rodenberger, R. F. Shi, M. Wu, N. W. Wang, Y. M. Cai, and A. F. Garito, Phy. Rev. A. 45, R4233-R4236 (1992).

49. D. C. Rodenberger, J. R. Heflin, and A. F. Garito, Nature 359, 309-311 (1992).

50. D. L. Andrews, S. Naguleswaran, and G. E. Stedman, Phys. Rev. A 57, 4925-4929 (1998).

51. A. D. Buckingham and P. Fischer, Phys. Rev. A 61, 035801 (2000).

52. G. E. Stedman, S. Naguleswaran, D. L. Andrews, and L. C. Dávila-Romero, Phys. Rev. A 63, 047801 (2001).

53. N. Bloembergen, Nonlinear Optics, Benjamin, New York, 1965.

54. D. C. Hanna, M. A. Yuratich, and D. Cotter, Nonlinear Optics of Free Atoms and Molecules, Springer, Berlin, 1979.

55. Y. R. Shen, The Principles of Nonlinear Optics, Wiley-Interscience, New York, 1984.

56. J. Berger, Eur. J. Phys. 11, 155-159 (1990).

57. L. C. Dávila-Romero, S. Naguleswaran, G. E. Stedman, and D. L. Andrews, Nonlin. Opt. 23, 191-201 (2000).

58. D. A. Kleinman, Phys. Rev. 126, 1977-1979 (1962).

59. R. Wortmann, P. Krämer, C. Glania, S. Lebus, and N. Detzer, Chem. Phys. 173, 99-108 (1993).

60. D. L. Andrews, Spectrochim. Acta 46A, 871-885 (1990).

61. D. L. Andrews, J. Phys. B, Atom. Mol. Phys. 13, 4091-4099 (1980).

62. D. L. Andrews and N. P. Blake, Phys. Rev. A 38, 3113-3115 (1988).

63. G. E. Stedman, Modern Nonlinear Optics, in M. Evans and S. Kielich (Eds.), Adv. Chem. Phys. 85 (Part 2), 489-543.

64. G. X. Cao and D. Zhu, Phys. Rev. B 51, 2418-2426 (1995).

65. N. Bloembergen, and Y. R. Shen, Phys. Rev. 133, A37-A49 (1964).

66. D. L. Andrews and W. J. Meath, J. Phys. B, Atom. Mol. Phys. 28, 4633-4641 (1993).

67. J. L. Oudar and D. S. Chemla, J. Chem. Phys. 66, 2664-2668 (1977).

68. B. Dick and G. Hohlneicker, J. Chem. Phys. 76, 5755-5760 (1982).

69. W. J. Meath and E. A. Power, Mol. Phys. 51, 585-600 (1984a).

70. W. J. Meath and E. A. Power, J. Phys. B, Atom. Mol. Phys. 17, 763-781 (1984b).

71. D. L. Andrews and P. Allcock, Chem. Phys. Lett. 231, 206-210 (1994).

72. A. J. Sadlej and P. W. Fowler, Chem. Phys. Lett. 245, 59-65 (1995).

73. S. Leasure and R. E. Wyatt, Chem. Phys. Lett. 61, 625-629 (1979).

74. M. A. Kmetic and W. J. Meath, Phys. Lett. A 108, 340-343 (1985).

75. A. E. Kondo, W. J. Meath, S. H. Nilar, and A. J. Thakkar, Chem. Phys. 186, 375-394 (1994).

76. P. Tran, W. J. Meath, B. D. Wagner, and R. P. Steer, J. Chem. Phys. 100, 4165-4170 (1994).

77. B. N. Jagatap and W. J. Meath, Chem. Phys. Lett. 258, 293-300 (1996).

78. K. A. Brueckner, Phys. Rev. 100, 36-55 (1955).

79. D. M. Bishop, J. Chem. Phys. 100, 6535-6542 (1994).

80. D. L. Andrews, L. C. Dávila Romero, and W. J. Meath, J. Phys. B, Atom. Mol. Opt. Phys. 32, $1-$ 17 (1999). 
81. G. Juzeliunas, L. C. Dávila Romero, and D. L. Andrews, work in progress.

82. Q. Song, C. Wan, and C. K. Johnson, J. Phys Chem. 98, 1999-2001 (1994).

83. P. K. Schmidt and G. W. Rayfield, Appl. Opt. 33, 4286-4292 (1994).

84. D. L. Andrews, P. Allcock, and A. A. Demidov, Chem. Phys. 190, 1-9 (1995).

85. P. Allcock, D. L. Andrews, S. R. Meech, and A. J. Wigman, Phys. Rev. A 53, 2788-2791 (1996).

86. D. L. Andrews and L.C. Dávila Romero, Proc. SPIE 4098, 284-293 (2000).

87. D. L. Andrews, Nonlin. Opt. 8, 25-32 (1994).

88. J. M. Nunzi, F. Charra, C. Fiorini, and J. Zyss, Chem. Phys. Lett. 219, 349-354 (1994).

89. C. Fiorini, F. Charra, and J.-M. Nunzi, J. Opt. Soc. Am. B 11, 2347-2358 (1994).

90. C. Fiorini, J.-M. Nunzi, F. Charra, I. D. W. Samuel, and J. Zyss, Int. J. Nonlin. Opt. Phys. 5, 653670 (1996).

91. P. Allcock and D. L. Andrews, J. Phys. B, Atom. Mol. Opt. Phys. 30, 3731-3742 (1997).

92. I. D. Hands, S. J. Lin, S. R. Meech, and D. L. Andrews, J. Chem. Phys. 109, 10580-10586 (1998).

93. I. D. Hands, S. J. Lin, S. R. Meech, and D. L. Andrews, Phys. Rev. A 62, 023807 (2000).

94. S. Lin, I. D. Hands, D. L. Andrews, and S. R. Meech, J. Phys. Chem. A 103, 3830-3836 (1999).

95. S. Lin, I. D. Hands, D. L. Andrews, and S. R. Meech, Opt. Commun. 174, 285-290 (2000).

96. S. Naguleswaran and G. E. Stedman, J. Phys. B, Atom. Mol. Opt. Phys. 29, 4027-4040 (1996).

97. D. L. Andrews and T. Thirunamachandran, J. Chem. Phys. 67, 5026-5033 (1977).

98. C. L. Tang and H. Rabin, Phys. Rev. B 3, 4025-4034 (1971).

99. Y. Sasaki and Y. Ohmori, Appl. Phys. Lett. 39, 466-468 (1981).

100. U. Österberg and W. Margulis, Opt. Lett. 11, 516-518 (1986).

101. R. H. Stolen and H. W. K. Tom, Opt. Lett. 12, 585-587 (1987).

102. N. B. Baranova and B. Ya. Zel'dovich, JETP Lett. 45, 717-720 (1987).

103. B. Ya. Zel'dovich and Yu. E. Kapitskii, JETP Lett. 51, 441-444 (1990).

104. T. J. Driscoll and N. M. Lawandy, J. Opt. Soc. Am. B 11, 355-371 (1994).

105. N. B. Baranova and B. Ya. Zel'dovich, J. Opt. Soc. Am. B, Opt. Phys. 8, 27-32 (1991).

106. S. R. Meech, private communication, 2000.

D. L. Andrews and P. Allcock, J. Phys. B, Atom. Mol. Opt. Phys. 30, 3731-3742 (1997). 